University of Louisville

ThinkIR: The University of Louisville's Institutional Repository

Electronic Theses and Dissertations

8-2018

\title{
Canonical nuclear factor-kB signaling in satellite stem cell homeostasis and function.
}

Alex R. Straughn

University of Louisville

Follow this and additional works at: https://ir.library.louisville.edu/etd

Part of the Molecular Biology Commons

\section{Recommended Citation}

Straughn, Alex R., "Canonical nuclear factor-kB signaling in satellite stem cell homeostasis and function." (2018). Electronic Theses and Dissertations. Paper 3043.

https://doi.org/10.18297/etd/3043

This Doctoral Dissertation is brought to you for free and open access by ThinkIR: The University of Louisville's Institutional Repository. It has been accepted for inclusion in Electronic Theses and Dissertations by an authorized administrator of ThinkIR: The University of Louisville's Institutional Repository. This title appears here courtesy of the author, who has retained all other copyrights. For more information, please contact thinkir@louisville.edu. 


\title{
CANONICAL NUCLEAR FACTOR-кB SIGNALING IN SATELLITE STEM CELL HOMEOSTASIS AND FUNCTION
}

\author{
By \\ Alex R. Straughn \\ B.S., University of Louisville, 2014 \\ M.S., University of Louisville School of Medicine, 2017
}

A dissertation submitted to the Faculty of the School of Medicine of the University of Louisville in Partial Fulfillment of the Requirements for the Degree of

Doctor of Philosophy in Anatomical Sciences and Neurobiology

Department of Anatomical Sciences and Neurobiology, University of Louisville, Louisville, Kentucky

August 2018 



\section{CANONICAL NUCLAR FACTOR-кB SIGNALING IN SATELLITE STEM CELL HOMEOSTASIS AND FUNCTION \\ By}

Alex R. Straughn, M.S.

A Dissertation Approved on

July 16, 2018

By the following Dissertation Committee:

Ashok Kumar, Ph.D.

Dissertation Director

Jennifer Brueckner-Collins, Ph.D.

Stanley D’Souza, Ph.D.

Charles Hubscher, Ph.D.

J. Patrick Moore, Ph.D. 


\section{DEDICATION}

To my parents for their unconditional love and support.

To my fiancé for helping to keep me sane. 


\section{ACKNOWLEDGMENTS}

I would like to acknowledge and thank first my mentor, Dr. Ashok Kumar, for all of the knowledge imparted to me.

Thank you to my committee members for their help and intellectual guidance: Dr. Jennifer Brueckner-Collins, Dr. Stanley D’Souza, Dr. Charles Hubscher, and Dr. J. Patrick Moore.

Dr. Sajedah Hindi, for her support and assistance throughout my time in the lab. Thank you for being you and helping me when I need it, especially after asking you the same question a million times.

Thank you to my other lab members, both current and previous: Dr. Yann Gallot, Dr. Guangyan Xiong, Dr. Kyle Bohnert, and Dr. Shuichi Sato. 


\section{ABSTRACT \\ CANONICAL NUCLEAR FACTOR-KAPPA B SIGNALING IN SATELLITE STEM CELL HOMEOSTASIS AND FUNCTION}

Alex R. Straughn

July 16,2018

Satellite cells are adult stem cells that are required for the regeneration of skeletal muscle following injury. However, the signaling mechanisms that regulate satellite stem cell homeostasis and function in adult animals remain less understood. Nuclear factorkappa $\mathrm{B}(\mathrm{NF}-\kappa \mathrm{B})$ is a major nuclear transcription factor that regulates the gene expression of a plethora of molecules involved in cellular proliferation, differentiation, survival, and the inflammatory immune response. NF-кB can be activated through a canonical or non-canonical pathway. However, the role of canonical NF- $\kappa \mathrm{B}$ signaling in the regulation of satellite stem cell function during skeletal muscle regeneration has not been yet investigated using genetic mouse models. In the present work, we demonstrate that physiological levels of activation of the canonical NF- $\kappa$ B pathway promotes satellite cell proliferation, survival, and differentiation. Satellite cell-specific inducible deletion of Inhibitor of Kappa B Kinase $\beta$ (IKK $\beta$ ), a critical kinase of the canonical NF- $\kappa B$ pathway, attenuates muscle regeneration in adult mice. Targeted ablation of IKK $\beta$ also reduces the number of satellite cells and their fusion to injured skeletal muscle of adult mice. Inhibition of canonical NF- $\mathrm{BB}$ pathway causes precocious differentiation of satellite cells both ex vivo and in vitro. We also found that siRNA-mediated knockdown of components 
of the canonical NF-кB pathway reduces the survival of cultured satellite cells.

Intriguingly, our results also demonstrate that supra-physiological activation of canonical $\mathrm{NF}-\kappa \mathrm{B}$ inhibits satellite stem cell function during skeletal muscle regeneration. Overexpression of a constitutively active mutant of IKK $\beta$ (IKK $\beta \mathrm{ca}$ ) in satellite cells attenuates initial stages of myofiber regeneration following injury. While not affecting their self-renewal, overexpression of IKK $\beta$ causes precocious differentiation of satellite cells. Furthermore, our results suggest that constitutive activation of canonical NF- $\mathrm{KB}$ pathway inhibits proliferation and reduces survival of satellite cells. Lastly, we found that inducible expression of IKK $\beta$ ca in satellite cells was insufficient to rescue the regenerative deficits observed in satellite cell-specific TAK1-knockout mice. Altogether,

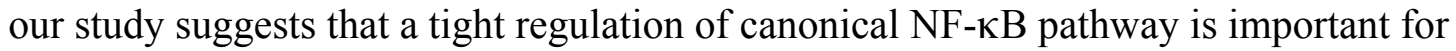
maintaining satellite cell pool and skeletal muscle regeneration. 
TABLE OF CONTENTS

PAGE

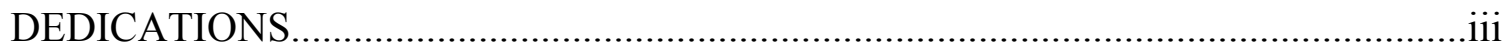

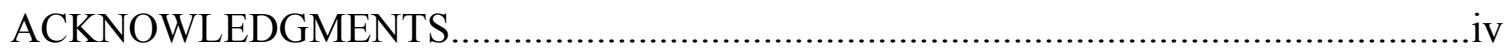

ABSTRACT .

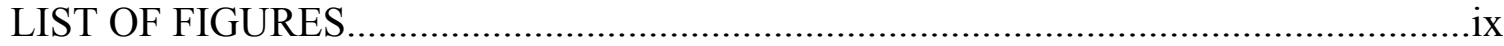

CHAPTERS

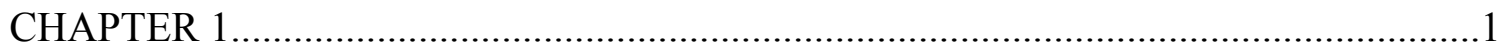

1.1 EMBRYOLOGICAL DEVELOPMENT OF SKELETAL MUSCLE...............1

1.2 SKELETAL MUSCLE INJURY AND REGENERATION PATTERN...........4

1.3 SATELLITE CELLS................................................................................6

1.4 SIGNALING MECHANISMS IN REGENERATION...................................

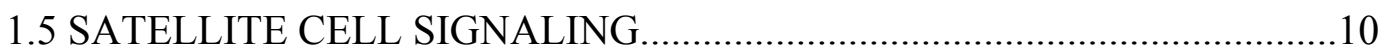

1.6 NF-KB SIGNALING........................................................................13

1.7 NF-KB SIGNALING IN MYOGENESIS AND SKELETAL MUSCLE

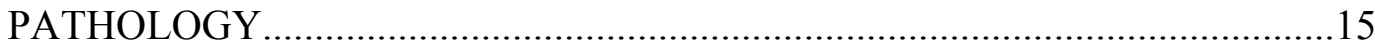

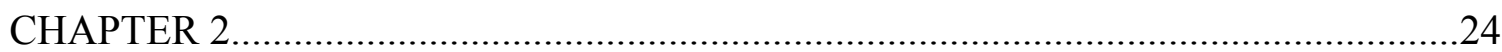

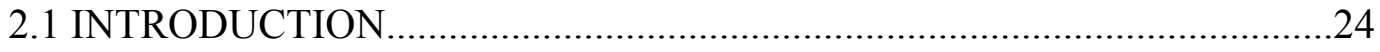

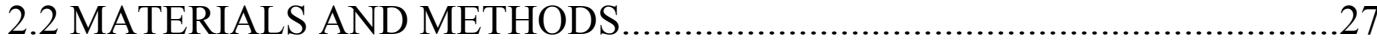

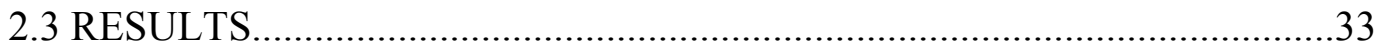

2.3.1 CANONICAL NF- $\kappa B$ SIGNALING IS ACTIVATED IN

SATELLITE CELLS AFTER SKELETAL MUSCLE INJURY...............33

2.3.2 SATELLITE CELL-SPECIFIC DELETION OF IKK $\beta$ DELAYS MYOFIBER REGENERATION IN ADULT MICE................................35

2.3.3 TARGETED INACTIVATION OF IKK $\beta$ DIMINISHES THE NUMBER OF NASCENT MYOFIBERS AND SATELLITE CELLS IN

REGENERATING MUSCLE OF ADULT MICE.....................................37

2.3.4 INHIBITION OF CANONICAL NF- $\kappa B$ SIGNALING REDUCES

THE PROLIFERATION OF SATELLITE CELLS....................................38

2.3.5 CANONICAL NF-אB SIGNALING PROMOTES THE SURVIVAL

OF SATELLITE CELLS.........................................................................

2.3.6 CANONICAL NF- $\kappa B$ SIGNALING PROMOTES THE SELF-

RENEWAL AND PREVENTS THE PRECOCIOUS

DIFFERENTIATION OF ACTIVATED SATELLITE CELLS.................40

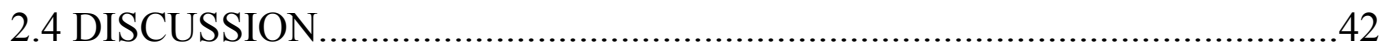

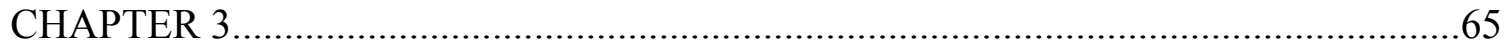




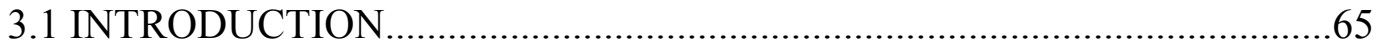

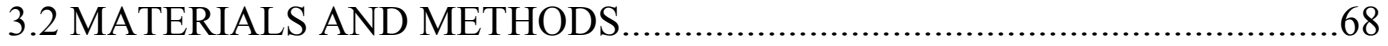

3.3 RESULTS.......................................................................................

3.3.1 CONSTITUTIVE ACTIVATION OF CANONICAL NF- $\kappa B$

SIGNALING IN SATELLITE CELLS ATTENUATES SKELETAL MUSCLE REGENERATION..............................................................74

3.3.2 OVEREXPRESSION OF IKK $\beta$ IN SATELLITE CELLS IMPEDES THE FORMATION OF NEW MYOFIBERS...........................................75

3.3.3 OVEREXPRESSION OF IKK $\beta$ IN SATELLITE CELLS LEADS

TO A REDUCTION SATELLITE CELL PROLIFERATION...................77

3.3.4 SATELLITE CELL-SPECIFIC OVEREXPRESSION OF CONSTITUTIVELY ACTIVE IKK $\beta$ PROMOTES CELL DEATH.........78 3.3.5 OVEREXPRESSION OF IKK $\beta$ IN SATELLITE CELLS CAUSES PRECOCIOUS DIFFERENTIATION OF SATELLITE CELLS WITHOUT AFFECTING THEIR SELF-RENEWAL...............................79

3.4 DISCUSSION

CHAPTER 4 101

4.1 INTRODUCTION 101

4.2 MATERIALS AND METHODS. 104

4.3 RESULTS 105

4.3.1 GENERATION OF INDUCIBLE SATELLITE-CELL SPECIFIC

TAK1 KNOCKOUT, IKK $\beta$ CONSTITUTIVELY ACTIVE MICE........105 4.3.2 FORCED EXPRESSION OF IKK $\beta$ IN TAK1-DEFICIENT SATELLITE CELLS YIELDS NO IMPROVEMENT IN REGENERATION IN VIVO. 106 4.4 DISCUSSION 107

CHAPTER 5 . 113

5.1 REVIEW OF DISSERTATION 113

5.2 SCIENTIFIC CONTRIBUTION AND FUTURE IMPLEMENTAT.............116

5.3 LIMITATIONS OF FUTURE IMPLEMENTATION...................................117

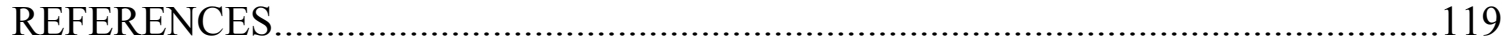

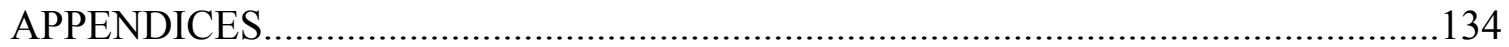

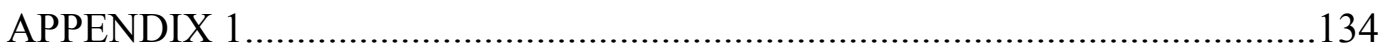

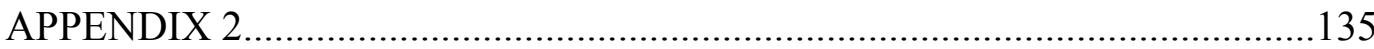

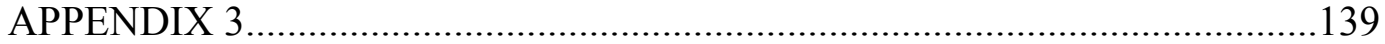

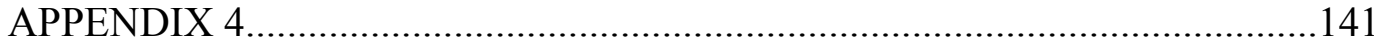

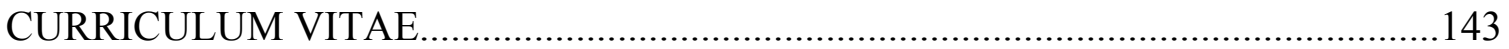




\section{LIST OF FIGURES}

FIGURE $\quad$ PAGE

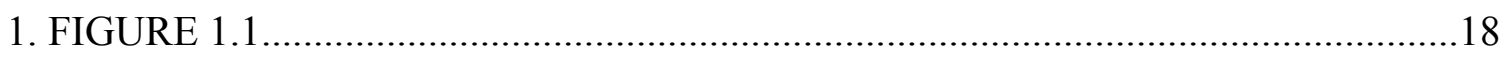

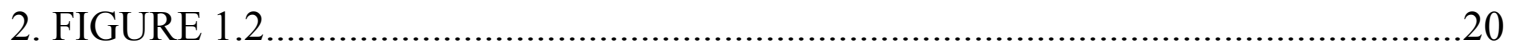

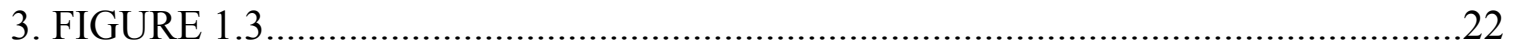

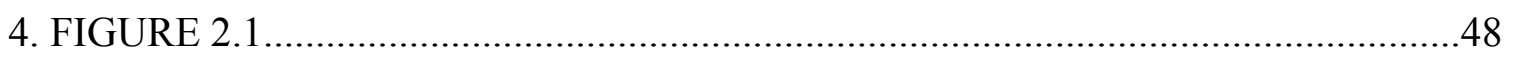

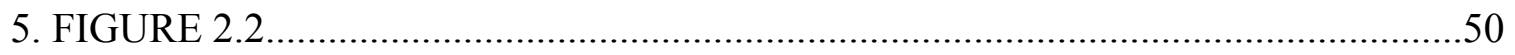

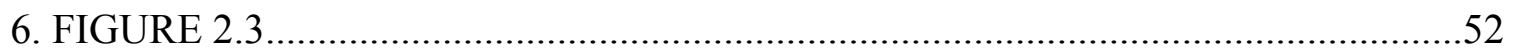

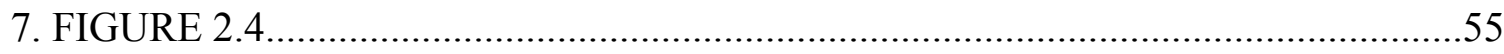

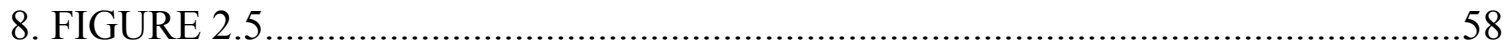

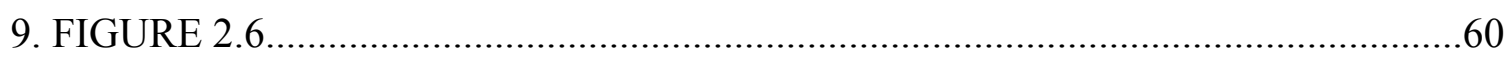

10. FIGURE 2.7

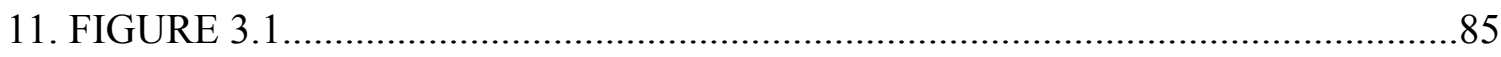

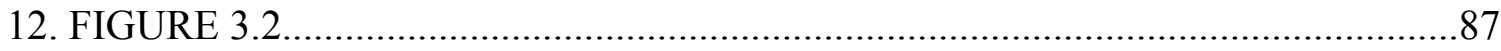

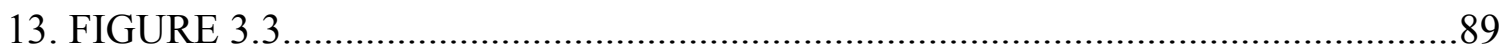

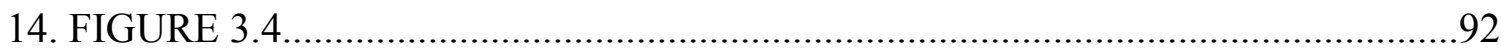

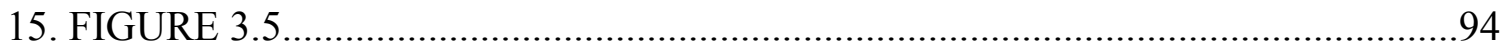

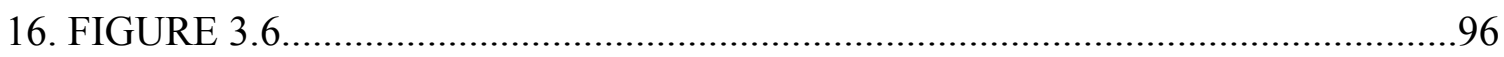

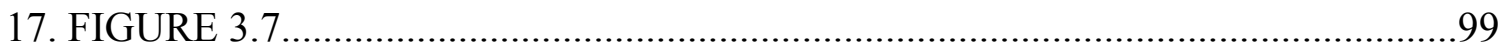

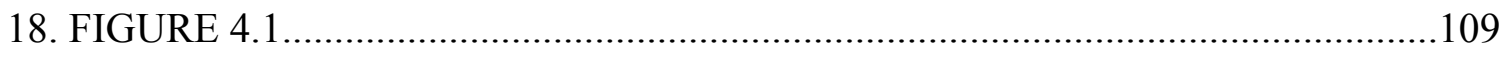

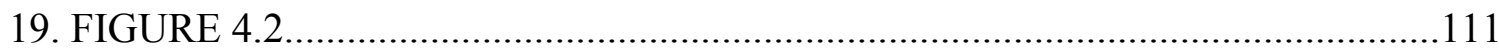




\section{CHAPTER 1}

\section{GENERAL INTRODUCTION}

\subsection{Embryological Development of Skeletal Muscle. Skeletal muscle is the most}

abundant tissue type in the human body that consists of multinucleated myofibers. Formation of skeletal muscle is a multistage process involving the hierarchical expression of various transcription factors and regulatory proteins. During the embryological development of skeletal muscle, mesodermal cells exit the primitive streak and proceed to migrate antero-laterally [1]. As the cells migrate out of the primitive streak, they spatially segregate to form both the axial and the paraxial mesodermal structures [1]. Various signaling molecules, such as: bone morphogenetic proteins (BMPs), the Wingless-related integration site (Wnt) pathway, and Noggin, a downstream target of Wnt signaling, are responsible for the organization and expansion of the paraxial mesoderm following the cellular migration from the primitive streak [2]. The paraxial mesodermal cells are responsible for the generation of somites, which are blocks of tissue that flank the neural tube [3]. The somites subsequently differentiate and give rise to the dermomyotome, and other embryologically-derived cell types. After the cells in the dermomyotome proliferate, they differentiate and give rise to the dermatome, which is the embryological tissue responsible for the generation of the dermis, and the myotome, 
which subsequently leads to the generation of muscle [2-4]. The myotome divides spatially into a hypaxial portion and an epaxial portion $[4,5]$. The hypaxial portion of the myotome gives rise to muscles of the anterior abdominal wall, as well as the skeletal musculature of the thoracic wall $[4,6]$. Proliferation and subsequent migration of cells in the epaxial portion of the myotome leads to the formation of the musculature of the trunk, as well as the extensor muscles of the neck $[4,6]$.

During and following the embryological orchestration of skeletal muscle patterning, the embryologically-derived skeletal muscle tissue signals for the production of more skeletal muscle, such that the musculoskeletal system can be fully developed. Myogenesis (the process of skeletal muscle formation) is required not only for development of skeletal muscle during embryogenesis, but it is also essential for the maintenance, growth, and repair of myofibers following injury [7]. The process of myogenesis can be divided into several stages to aide in understanding the molecular mechanisms orchestrating the formation of skeletal muscle. Myogenesis starts with the process of delamination and ends with the formation of specific skeletal muscles. Delamination is initiated by the genetic factor Paired-box 3 (Pax3), which mediates the transcription of protein tyrosine kinase Met (c-Met) [8]. Pax3 is expressed in hypaxial cells that are migrating, but is not expressed in cells that lead to development of the facial muscles [9]. c-Met-mediated signaling initiates the generation of lamellipodia in the nascent myoblasts, thereby conferring them the capability to migrate to its future resident area $[10]$.

The next component of myogenesis is migration, which orchestrates the organization of the developing dorsal forelimb [11]. Ladybird Homeobox 1 (LBX1) is 
critical for the development of the dorsal forelimb post-delamination, as signaling through LBX1 stimulates the proliferation of myoblasts $[4,8,12]$. The proliferation of myoblasts in the developing dorsal forelimb in turn populates this region with skeletal muscle progenitors, such that further limb development can occur [12]. The signaling molecule c-Met continues to be upregulated during this stage of myogenesis and continues to orchestrate the migration of the newly generated myoblasts $[4,8,10]$.

After the newly derived myoblasts have migrated to their target region, the embryologically derived myoblasts will rapidly undergo proliferation and eventually will be signaled to differentiate, thereby completing the next two components of myogenic signaling. The myoblasts are signaled to induce gene expression of various myogenic regulatory factors (MRFs) [13]. The first MRF to be expressed is Myogenic factor 5 (Myf-5) on embryological development day 8 to facilitate the proliferation of myoblasts [13]. Myogenin and Myoblast determination protein $1(\mathrm{MyoD} / \mathrm{MyoD} 1)$ are the next MRFs to be expressed to initiate the differentiation of the expanded population of myoblasts [14, 15]. MyoD also inhibits self-renewal of myogenic cells [16].

With the expression of MyoD, the myoblasts have entered the myogenic stage of determination. In this facet of myogenesis, Pax7 is down-regulated and MyoD is concurrently up-regulated [17]. The myoblasts subsequently differentiate into myocytes as a result of the expression of MyoD and other signaling molecules [13, 18-20]. Following this differential signaling paradigm, the myocytes are signaled to fuse with each other to form primary myotubes, which will subsequently fuse with more myocytes in a process termed secondary fusion that ultimately culminates in the generation of mature myofibers (Figure 1.1) [13, 18-20]. 
The expression pattern of Myf-5 is only transient, whereas MRF4, Myogenin, and MyoD are expressed in perpetuity [15]. As the developing embryo gestates and undergoes the myogenic process, the signaling molecules LBX1 and Mesenchyme Homeobox 2 (Mox2) facilitate specific muscle formation in conjunction with numerous other proteins, thereby finalizing the process of myogenesis $[4,8,21]$. To allow mature development of the musculoskeletal system, LBX1 continues to stimulate the proliferation of myoblasts [12] and Mox2 signaling is regulated in a spatiotemporal fashion to allow specific appendicular muscle formation [22].

1.2 Skeletal Muscle Injury and Regeneration Pattern. Despite skeletal muscle being a terminally differentiated cell type, it has the amazing capability to repair itself following injury due to a self-sustaining population of undifferentiated precursor cells, termed satellite cells [23]. After an acute injury to skeletal muscle, a multitude of signaling cascades are activated from the damaged muscle and surrounding cells to assist in the recovery/regeneration of the injured skeletal muscle [23]. The injury and subsequent regeneration process, termed regenerative myogenesis, follows a stereotypical pattern that is mediated primarily by satellite cells [24]. By day one post-injury, a necrotic muscle environment is generated and there is a robust invasion of the injured tissue by numerous inflammatory cells [24]. By day three post-injury, an abundance of mononuclear cells are recruited to the injured skeletal muscle [24]. Following this, the production of myoblasts is signaled to facilitate a repair of the damaged microenvironment [24]. In tandem, the myoblasts will proliferate, differentiate, and subsequently fuse with each other and pre-existing myofibers while the infiltrating 
immunocytes are cleared [24]. Three weeks post-injury, the invading inflammatory cells have been mostly cleared due to phagocytic macrophage activity to the point that the injured skeletal muscle tissue has returned to a normal physiological appearance, with the exception of the injured myofibers being centrally multinucleated as a byproduct of myoblast fusion [24].

As briefly alluded to, several immunocytes play a role in the regenerative process post-injury [25]. Following an acute skeletal muscle injury, the sarcolemma of skeletal muscle is disrupted, which leads to an extravasation of various myokines (chemokines released from skeletal muscle) that aide in the recruitment of immune cells to the injured microenvironment [26-28]. Several adaptor signaling proteins, such as Myeloid Differentiation Primary Response 88 (MyD88), TIR-domain-containing Adaptorinducing Interferon- $\beta$ (TRIF), Translocation Associated Membrane Protein (TRAM), and TIR-domain-containing Adaptor Protein (TIRAP), mediate Toll-like Receptor (TLR) signaling cascades that result in the activation of NF- $\mathrm{kB}$, Interferon Regulatory Factors, and other signaling pathways [26, 29]. TLRs allow for the recognition of DamageAssociated Molecular Patterns (DAMPs) and Pathogen-Associated Molecular Patterns (PAMPs), making them ideally suited for signaling during regenerative myogenesis [26]. TLR-mediated signaling pathways also allow for the production of various myokines and induce the generation of pro-inflammatory cytokines [26-29].

Post-injury, various immunocytes are recruited from peripheral tissues to the damaged skeletal muscle microenvironment. Neutrophils are the first cell type to be recruited and typically infiltrate the skeletal muscle within an hour following injury and increase in number peaking around 24-hours post-injury [27]. After the skeletal muscle is 
infiltrated by neutrophils, phagocytic macrophages, initially responsible for phagocytosis of cellular debris, invade the injured skeletal muscle and peak in number 48-hours postinjury [27]. The macrophages are signaled to express various monocyte chemoattractant proteins [30]. Peripherally, monocytes are being generated in the bone marrow due to signaling from the damaged skeletal muscle [27]. These monocytes are recruited to the site of injury due to the macrophage-mediated expression of monocyte chemoattractant proteins, where they will subsequently differentiate into phagocytic M1 macrophages or into non-phagocytic M2 macrophages [27, 30]. The M1 macrophages have a proinflammatory role and are responsible for mediating the subsequent recruitment of more M1 macrophages, as well as M2 macrophages [31]. The M1 macrophages provide an initial cellular benefit from the recruitment of non-resident immunocytes, but prolonged expression and activity of the phagocytic macrophages can worsen the degree of inflammation and injury in the damaged skeletal muscle microenvironment [31, 32]. The M2 macrophages arise during a relatively later stage during the process of regeneration and mediate a transition from a protectant pro-inflammatory phase into an antiinflammatory phase that allows for the facilitation of repair [32].

1.3 Satellite Cells. Skeletal muscle contains a group of undifferentiated precursors that are maintained in a quiescent state, termed satellite cells. Under uninjured conditions, the satellite cells reside between the basal lamina and the sarcolemma [23]. Quiescent, or inactive, satellite cells can easily be identified by their expression pattern of certain myogenic markers, such as: Pax7, CD34, and M-Cadherin [33]. After sustaining an injury, the previously inactive satellite cells are rapidly activated to facilitate repair of 
damaged skeletal muscle (Figure 1.2) [34]. A population of these satellite cells tentatively commits to the myoblast lineage and expresses various myogenic markers, such as: $\alpha 7$-integrin, Myf-5, MyoD, and Pax7 [33]. Pax7, in conjunction with other signaling molecules, such as MyoD, determine the fate of the satellite cells $[17,35]$. Activated satellite cells destined to facilitate with the repair process will usually undergo an up-regulation of MyoD [17]. These satellite cells will simultaneously up-regulate MyoD and down-regulate $\operatorname{Pax} 7$ to the point that they become $\mathrm{Pax}^{-} / \mathrm{MyoD}^{+}$and have committed to the myogenic lineage to facilitate repair of injured myofibers [17]. Alternatively, satellite cells can self-renew their population through a down-regulation of MyoD and undergo asymmetric cell division [17]. This replenishment of the satellite cell pool through asymmetric division, common in numerous stem cell types, provides a means for skeletal muscle to repair future injuries by maintaining a resident population of the undifferentiated precursor cell type [33].

1.4 Signaling Mechanisms in Regeneration. It is now evidenced that a number of signaling pathways are activated in skeletal muscle of adults following injury. The majority of the signaling mechanisms are activated in injured myofibers and satellite stem cells [23]. However, several other cell types do play a role in the repair of skeletal muscle. For example, immunocytes that infiltrate the muscle following necrotic injury initiate signaling pathways that orchestrating changes in the cellular immune response [23]. There is also evidence for non-muscle adult stem cells, such as certain hematopoietic stem cells, being recruited to assist in skeletal muscle regeneration [36]. 
Below, I discuss a group of signaling mechanisms that mediate various facets of skeletal muscle regeneration [23].

Several fibroblast growth factors (FGFs) have been implicated in regenerative myogenesis, although some with seemingly contradictory results [23, 37-40]. FGF-6 has a muscle specific expression pattern and signaling increases post-injury [37]. Prior reports indicate that the skeletal muscle of mice lacking FGF-6 is still capable of undergoing regeneration despite its reported role in cellular signaling following an acute muscle injury [38]. FGF-2 is a stimulus for myoblast activation [39] and has also been shown to increase the proliferation of satellite cells, thereby improving muscle regeneration [40].

On similar lines, Insulin-like Growth Factors (IGFs) also play important role in the regulation of myogenesis [41-43]. IGF-I and IGF-II lead to changes in the gene expression patterns of various MRFs that regulate numerous facets of the myogenic process [43]. IGF-I results in skeletal muscle hypertrophy through the activation of satellite cells and their subsequent proliferation, as well as through stimulating an increase in the rate of protein synthesis in pre-existing skeletal muscle [44-47]. IGF-II activity, mediated by mechanistic target of Rapamycin (mTOR) signaling, results in changes to IGF-II promoter 3 transcriptional activity, conferring an enhanced differentiation of the previously generated myoblasts signaled through IGF-I [48].

Conversely, the Transforming Growth Factor- $\beta$ (TGF- $\beta$ ) family of cytokines inhibits regenerative myogenesis [49]. Members of the TGF- $\beta$ family have been shown to inhibit proliferation and differentiation of myoblasts within the context of regenerative myogenesis $[50,51]$. The impairment to skeletal muscle regeneration mediated by TGF- 
$\beta 1$ occurs through down-regulation of basic fibroblast growth factor (bFGF) and IGF1 signaling, which causes a decrease in myoblast proliferation [50]. Another member of the TGF- $\beta$ family, Myostatin (also known as Growth and Differentiation Factor-8), has been implicated in the inhibition of regenerative myogenesis [52]. Prior reports have shown that the skeletal muscle of Myostatin-deficient mice displays both hyperplasia and hypertrophic growth of myofibers within skeletal muscle [52]. Further, the levels of Myostatin have been found to be up-regulated following necrotic muscle injury [53]. It has been suggested that Myostatin acts as a chemoattractant thereby mediating TGF- $\beta$ signaling and the recruitment of macrophages in the injured muscle environment [53]. Hepatocyte Growth Factor (HGF), also known as Scatter Factor, is a critical growth factor with respect to tissue regeneration [54]. HGF transcript levels are increased during the early stages of injury in a manner proportional to the severity of skeletal muscle injury [55-57]. Other studies have shown that HGF activates quiescent satellite cells and increases their proliferation, while simultaneously inhibiting their differentiation, thereby facilitating the early stages of regenerative myogenesis [56]. Downstream signaling through HGF and the canonical NF- $\mathrm{kB}$ pathway act antagonistically upon each other to either facilitate the differentiation or the proliferation of myoblasts, respectively [58]. Later stages of regenerative myogenesis show a decreased expression of HGF, possibly to allow the proliferating satellite cells to differentiate and fuse with injured myofibers [56].

Leukemia Inhibitory Factor (LIF) is a member of the Interleukin-6 cytokine family and has been shown to play a very specific role in regenerative myogenesis [59]. LIF appears to augment myoblast proliferation via the activation of Janus Kinase 2 
(JAK2)-Signal Transducer and Activators of Transcription Protein 3 (STAT3) signaling, but does not affect the differentiation or subsequent fusion of myoblasts [60, 61]. Despite its structural homology to LIF, IL-6 does not appear to affect regenerative myogenesis $[62,63]$. Despite no definitive role in regenerative myogenesis, IL-6 regulates protein synthesis, skeletal muscle adaptation to contraction, and cancer-induced muscle atrophy [64].

1.5 Satellite Cell Signaling. Satellite cells are inextricably linked to regenerative myogenesis. Indeed, satellite cells are connected to a multitude of different signaling pathways that exhibit crosstalk between each other, thereby resulting in a complex signaling network within the satellite cells [65]. Some of these pathways signal for the self-renewal of satellite cells $[33,35,66,67]$, whereas others promote escape from quiescence and progression into myogenic lineage [68-71]. Asymmetric division of the satellite cells is a hallmark of self-renewal [33], whereas the symmetric expansion of satellite cells is indicative of a commitment to the myogenic lineage [17, 35]. Discussed below are various signaling pathways that modulate whether the satellite cells will undergo self-renewal or whether they will activate and subsequently differentiate to facilitate the repair of injured skeletal muscle.

One of the pathways involved with the self-renewal of satellite cells is the Notch signaling cascade. This pathway is initiated when a Notch ligand, such as Delta-like (DLL) 1 or 4 or Jagged 1 or 2, binds to an adjacent cell that has a Notch Receptor, Notch 1-4 [66]. The Notch receptor undergoes proteolytic cleavage, thereby freeing the Notch Intracellular Domain (NICD) to translocate to the nucleus [72]. NICD binds to 


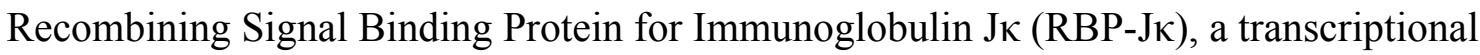
repressor. Binding of NICD results in a conformational change in RBP-J $\kappa$, resulting in the formation of a transcriptional activator [72]. Basal levels of Notch activity have been shown to be critical in the maintenance of quiescent satellite cells [73]. Published reports demonstrate that a subset of quiescent satellite cells express a high level of Notch activity [35]. Other studies showed that the forced expression of Notch signaling was sufficient to restore the regenerative capability of aged myofibers, lending further support to its critical role in the maintenance of pool of satellite cells [74].

The signaling pathways of four mitogen-activated protein kinases (MAPKs)— extracellular signal-regulated kinase 1/2 (ERK1/2), c-Jun N-terminal kinase (JNK), p38, and ERK5 are among the most well-characterized cell-signaling pathways that are activated in response to various extracellular stimuli and regulate pluripotent cellular function in almost all cell types. Signaling through Angiopoietin 1, and its receptor Tie-2, is mediated through the ERK1/2 pathway and has been shown to play an anti-myogenic role that results in the quiescence of satellite cells [67]. The ERK1/2 signaling pathway is activated by various cytokines that act to orchestrate proliferation by regulating progression through the cell cycle and apoptosis [75-79]. Tie-2 was shown to be expressed in quiescent satellite cells and the expression decreased with the activation and subsequent differentiation of satellite cells [67]. When the ERK1/2 pathway is silenced in satellite cells, they begin to up-regulate numerous pro-myogenic markers that cause the satellite cells to undergo proliferative expansion, followed thereafter by their differentiation [67]. 
The JNK signaling pathway is inextricably linked to satellite cells and the process of myogenesis [80]. JNK is activated by various growth factors, pro-inflammatory cytokines, as well as environmental stress signals, and is notable for its role in cellular death [81-83]. However, aside from its role in the initiation of apoptosis, JNK has been shown to regulate cellular proliferation and differentiation [80-83]. Published reports suggest that during the later stages of myogenesis, the JNK pathway is severely downregulated [80]. When this pathway is active in satellite cells, differentiation is inhibited and the satellite cells are able to self-renew or remain in a quiescent state depending on the context of cellular signaling [80].

Conversely, activation of the p38 MAPK signaling pathway results in a loss of satellite cell self-renewal [69]. The p38 MAPK signaling pathway is activated in response to various cytokines and environmental stress signaling and results in a differentiation of cells or in their apoptotic and autophagic cell death [84-87]. Throughout myogenesis, p38 MAPK signaling is activated and results in cellular differentiation by inducing cells to withdraw from the cell cycle [88-91]. Previous studies have found that when the JNK pathway is inhibited, the p38 MAPK pathway is up-regulated [80]. Satellite cells from aged mice show an up-regulation of the p38 MAPK pathway which may be a reason for the inhibition of asymmetric division of satellite cells [69].

Recently, the Wnt7a/Fzd7 planar cell polarity pathway (non-canonical Wnt signaling) has been found to drive the symmetric division of satellite cells to facilitate regenerative myogenesis [68]. A portion of the satellite cells (i.e. $\mathrm{Pax}^{+} / \mathrm{MyoD}^{+}$) express the alternative Wnt signaling receptor Frizzled 2 (Fzd7) [68]. In non-canonical Wnt signaling, Wnt activates the small GTPases Rho and Rac after receptor activation [68]. 
Rho and Rac cause a rearrangement of the cytoskeleton that results in a symmetric division of the satellite cells [92]. Skeletal muscle containing Fzd7-deficient satellite cells show a marked decrease in regenerative capacity, whereas $\mathrm{Fzd}^{+}$satellite cells demonstrate a robust ability to facilitate regenerative myogenesis [92].

The JAK-STAT signaling pathway causes activation of satellite cells and inhibits quiescence [71]. In various cell types, JAK/STAT signaling pathway has been shown to induce proliferation, migration, and differentiation. Prolonged activation of this pathway also causes cell death through apoptosis [93-97]. When receptors that are associated with JAK tyrosine kinases are activated, they multimerize as hetero- and homodimers and activate JAK signaling due to the multimerization bringing JAK proteins into close proximity [94]. Activation of the JAK proteins causes a phosphorylation of, amongst other things, the STAT transcription factors $[94,96]$. Activated STATs translocate to the nucleus, dimerize, and then bind to various regulatory sequences to alter transcription of target genes [97]. Reports have shown that knockdown of Jak2 or Stat3 result in an increase in the symmetric division of satellite cells and an increase in satellite cell ability to differentiate and contribute to regenerative myogenesis [71]. Upon activation, Stat3 promotes the differentiation of myoblasts through an up-regulation of MyoD [98]. It has been previously reported that the satellite cells in skeletal muscle of aged humans' exhibit increased expression of various components of JAK-STAT pathway and a concomitant impediment of satellite cell quiescence [99].

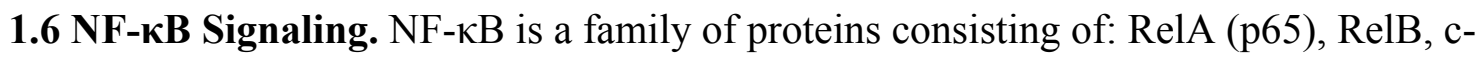
Rel, the canonical NF- $\mathrm{\kappa B}$ precursor $\mathrm{p} 105$ and spliced form $\mathrm{p} 50$, and the non-canonical 
NF- $\mathrm{BB}$ precursor p100 and spliced form p52, which form hetero- and homodimers [100]. $\mathrm{NF}-\kappa \mathrm{B}$ resides in its inactive state in the cytoplasm as a heterotrimer consisting of $\mathrm{p} 50$, $\mathrm{p} 65$, and $\mathrm{I} \kappa \mathrm{B} \alpha$ subunits. On activation of the complex, I $\kappa \mathrm{B} \alpha$ sequentially undergoes phosphorylation, ubiquitination, and degradation, thus releasing the p50-p65 heterodimer for translocation to the nucleus. NF- $\mathrm{BB}$ can be activated by pro-inflammatory signals, such as TNF- $\alpha$, leading to the activation of the canonical NF- $\mathrm{BB}$ pathway (Figure 1.3) [100]. In the canonical pathway, inhibitor of $\kappa \mathrm{B}(\mathrm{I} \kappa \mathrm{B})$ is phosphorylated and degraded by

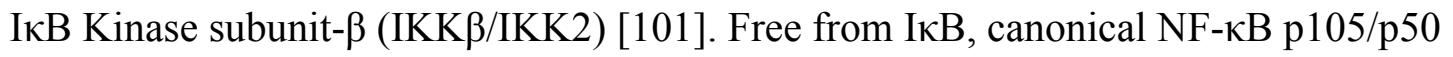
translocates to the nucleus where it stimulates gene expression of a wide variety of molecules [101]. Conversely, NF- $\kappa \mathrm{B}$ can be activated in an IKK $\beta$-independent fashion, termed the alternative or non-canonical NF- $\kappa$ B pathway (Figure 1.3) [100]. In contrast, activation of the non-canonical NF- $\kappa$ B pathway requires the activation of NF- $\mathrm{BB}-$ inducing kinase (NIK) and IKK $\alpha$, leading to the phosphorylation and proteolytic processing of the p100 subunit to generate p52 [102]. The canonical pathway primarily utilizes a heterodimer of RelA and p105/p50 [101], whereas the non-canonical pathway involves the translocation of p52/RelB dimers to the nucleus [102].

TGF $\beta$-activated kinase 1 (TAK1/MAP3K7) is a member of the MitogenActivated Protein Kinase Extracellular Signal-Regulated Kinase (MEK) Kinase family that activates numerous downstream signaling pathways [103]. TAK1 is normally bound to accessory protein TAK1-binding protein 1 (TAB1), but upon stimulation, it also interacts with TAB2 and TAB3 [104]. Pro-inflammatory stimuli activate TAK1 through activation of several upstream molecules, a process that also involves K63-linked polyubiquitination [105]. This polyubiquitination is driven by the E2 ligase, Ubc13, or by 
one of the E3 ligases, such as Tumor Necrosis Factor (TNF) Receptor (TNFR) Associated Factor 6 (TRAF6) [106]. TAB2 and TAB3 have a preferential binding affinity to K63-linked polyubiquitin chains [106]. Thus, once TAK1 is polyubiquitinated, TAB2 and TAB3 bind leading to the enzymatic activation of TAK1 $[106,107]$. Activated TAK1 causes the phosphorylation of several downstream signaling proteins, including IKK $\beta$ that leads to the activation of canonical NF- $\mathrm{kB}$ pathway [107].

Activation of TAK1 in response to cellular stress can lead to the initiation of either anti- or pro-apoptotic signaling cascades [107]. TAK1 activates anti-apoptotic pathways by signaling to NF- $\mathrm{kB}$ and JNK, whereas it will utilize the activation of caspases to induce pro-apoptotic pathways [107]. Although TAK1 facilitates both a proapoptotic and an anti-apoptotic state, my lab has shown that inactivation of TAK1 leads to increased oxidative stress and satellite cell death primarily through necroptosis [65].

\subsection{NF-кB Signaling in Myogenesis and Skeletal Muscle Pathology. Within the} context of skeletal muscle myogenesis, there is a bit of a dichotomy between the role of canonical [108] and non-canonical NF-kB signaling [109]. The canonical pathway is activated in proliferating myoblasts [108]. Indeed, activation of canonical pathways inhibits differentiation of myoblasts into myotubes [108]. Canonical NF-кB signaling causes down-regulation of $\mathrm{MyoD}$, which is required to escape proliferation and progress further into myogenesis [110]. Conversely, the non-canonical NF-kB pathway is active when myoblasts are starting to undergo fusion to form myotubes [109]. It is also involved in mitochondrial biogenesis, which allows the growing myotubes to meet their ATP needs [109]. Forced expression of the canonical NF- $\mathrm{kB}$ pathway has been shown to cause 
progressive muscle atrophy [111], whereas an inhibition can lead to a reduction of inflammatory and fibrotic changes that facilitate muscle repair [112].

The canonical NF- $\mathrm{KB}$ pathway has been studied extensively in myoblasts and myofibers $[108,109,111,113-116]$. However, the role of canonical NF- $\mathrm{KB}$ signaling in the regulation of satellite cell function during regenerative myogenesis has not been yet investigated. Recently, the role of a few upstream molecules of the canonical NF- $\mathrm{KB}$ signaling pathway has been investigated in satellite cells $[65,117]$. Work from my lab has shown that TRAF6 plays a critical role during regenerative myogenesis and for the homeostasis of satellite cells [117]. It was demonstrated that the deletion of TRAF6 in satellite cells led to a dramatic deficit in skeletal muscle regeneration [117]. TRAF6 is required for the activation of the JNK $1 / 2$ and ERK $1 / 2$, which subsequently activate c-Jun transcription factor [117]. Activated c-Jun binds to the promoter region and induces gene expression of Pax7. The TRAF6/c-Jun signaling axis also lead to down-regulation of miR-1 and miR-206, which normally promote the differentiation of myoblasts [117]. My lab has also shown that TAK1 is critical for satellite cell functionality and maintenance [65]. Prior work from my lab showed that when TAK1-deficient satellite cells precociously differentiate and rapidly deplete the satellite cell pool [65]. Further, it was shown that TAK1 mediates the activation of JNK signaling, which is necessary to prevent precocious differentiation of the satellite cell pool and to prevent oxidative stress [65].

Although not specifically the focus of my thesis, the canonical NF- $\mathrm{kB}$ signaling pathway is involved in a multitude of muscle pathologies and age-related changes associated with skeletal muscle [118]. It has been shown that NF-kB signaling is activated in various inflammatory myopathies, such as Duchenne muscular dystrophy, 
polymyositis, and dermatomyositis [119]. The general trend in these myopathies is that the canonical NF- $\mathrm{BB}$ signaling pathway is over-activated, which exacerbates the inflammation associated with these conditions and increases cellular oxidative stress $[119,120]$. In certain alveolar rhabdomyosarcoma substrains, the canonical NF-кB-YY1miR-29 signaling axis is disturbed [121]. Along similar lines, the age associated muscle wasting, or sarcopenia, has been shown to be partially mediated by increased activation of the canonical NF- $\kappa \mathrm{B}$ signaling pathway [111].

Ultimately, the specific role of canonical NF- $\mathrm{BB}$ signaling in satellite cells remains poorly studied. Within the context of regenerative myogenesis, the role of canonical NF- $\kappa$ B signaling in satellite cells is completely unknown. Thusly, we sought to investigate the role of canonical NF- $\mathrm{B}$ signaling in satellite cells. The experiments detailed in this thesis work to further our understating of: NF- $\kappa \mathrm{B}$ signaling, satellite cell homeostasis, and the process of muscle regeneration. Through the generation of: an inducible satellite cell-specific IKK $\beta$ knockout mouse, an inducible satellite cell-specific IKK $\beta$ overexpression mouse, and a concurrent inducible satellite cell-specific TAK1 knockout-IKK $\beta$ overexpression mouse, here we investigate the role of canonical NF- $\kappa \mathrm{B}$ signaling in satellite cell proliferation, differentiation, and survival during regenerative myogenesis. Our results demonstrate that tight regulation of IKK $\beta$ is essential for the proliferative and regenerative capabilities of satellite cells, both in vivo and in vitro. 


\section{FIGURE 1.1}

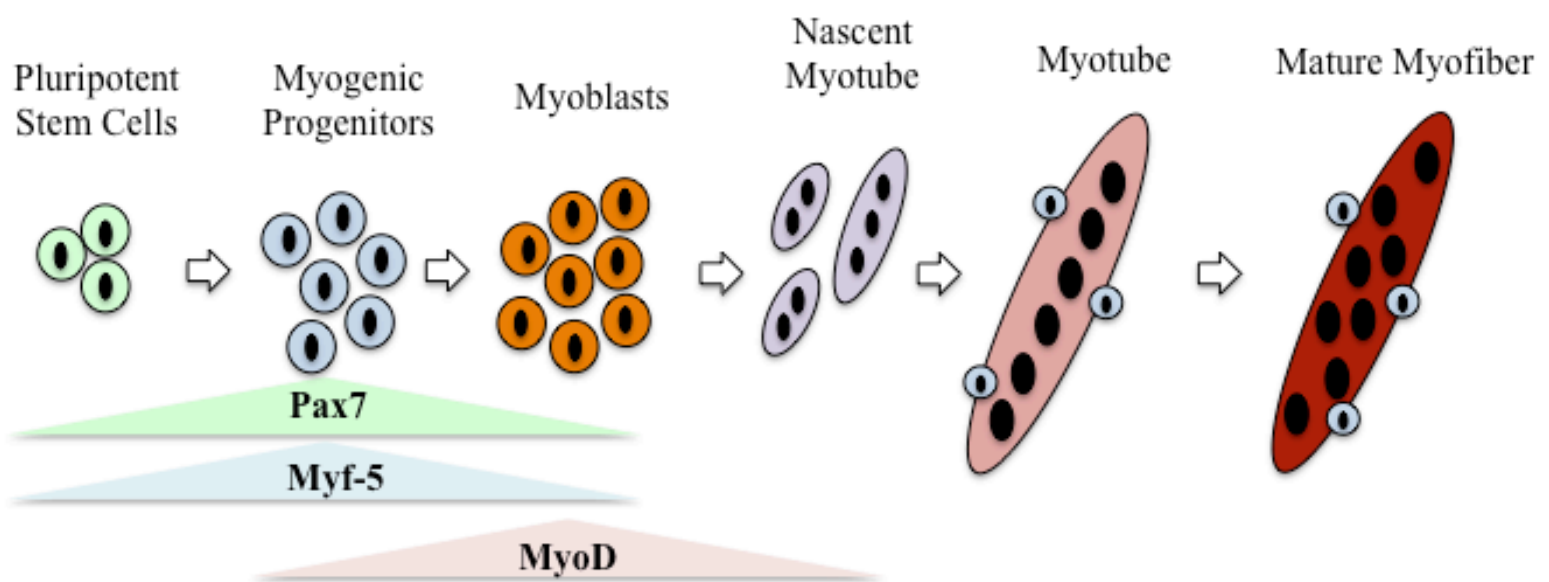

Myogenin

MRF4 
Figure 1.1 Myogenesis. Schematic representation of the process of myogenesis. Myogenic regulatory factors orchestrate the progression from the pluripotent stem cell state to the adult myofiber. Pax7 is a marker for the early myogenic stem cells and progenitors. Myf-5 is the first MRF to be activated, and is followed sequentially by MyoD, Myogenin, and MRF4. Eventually, myosin heavy chain, a marker of mature skeletal muscle, begins to be expressed. 


\section{FIGURE 1.2}
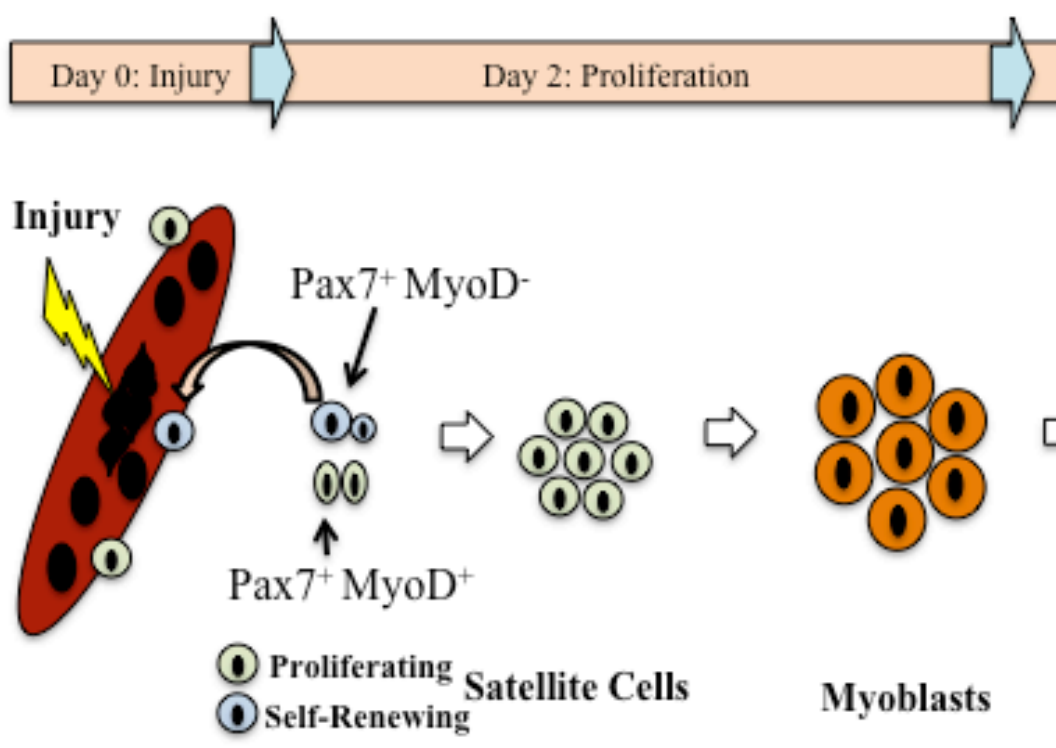

Days 3 \& 4: Differentiation

Pax7

Myf-5

MyoD

Myogenin 
Figure 1.2 Satellite cell activation post-injury. Schematic representation of satellite cell activation following skeletal muscle injury. Satellite cells get activated and begin to express MyoD, leading to their proliferation through symmetric cell division. Eventually, through hierarchical MRF signaling, the satellite cells differentiate and then fuse with the regenerating myofibers. Alternatively, the satellite cells down-regulate MyoD and undergo asymmetric cell division to self-renew their stem cell population and then return to quiescence. 


\section{FIGURE 1.3}

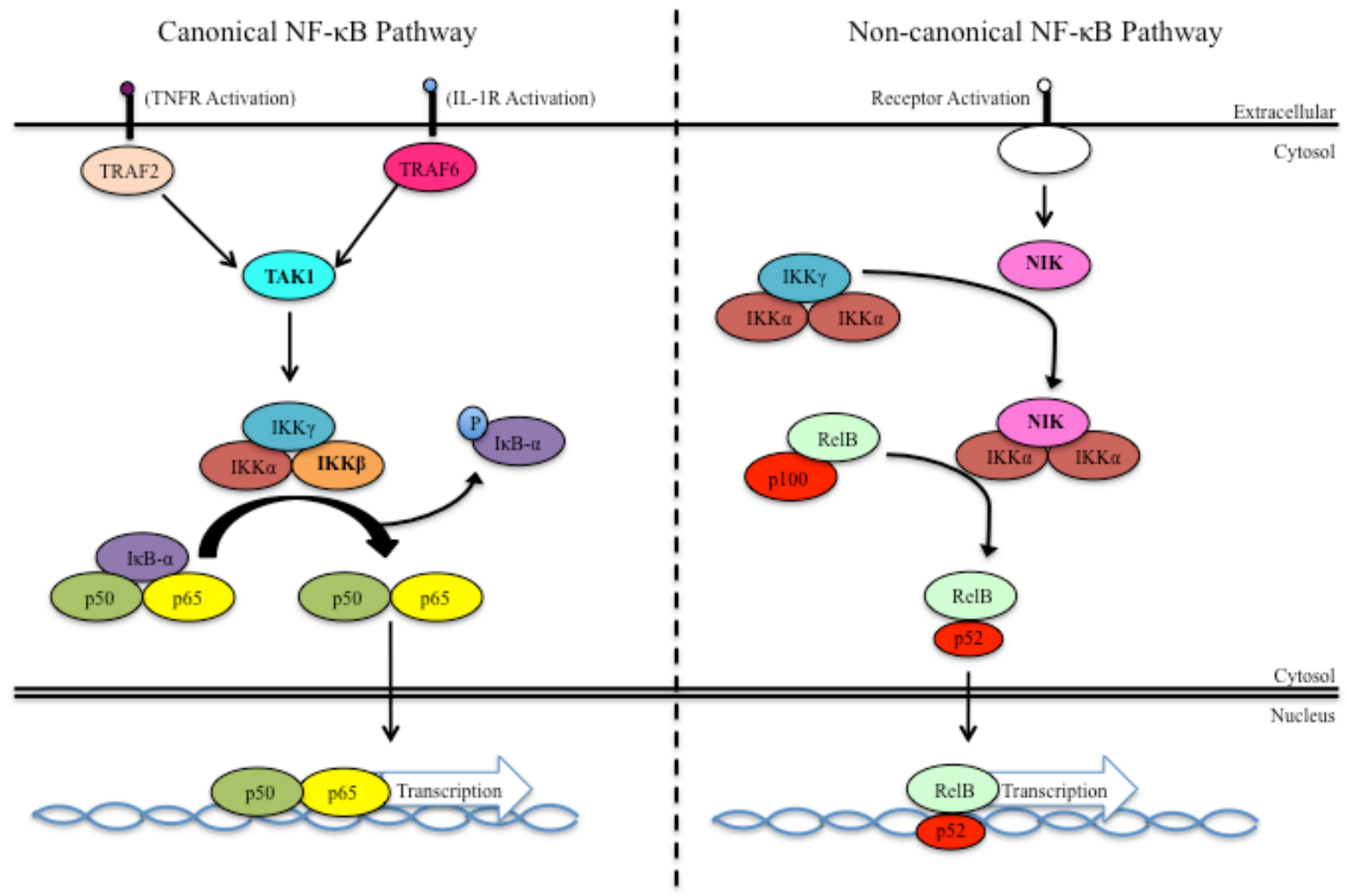


Figure 1.3 Canonical and non-canonical NF-кB signaling. Schematic representation of the two NF- $\kappa$ B signaling pathways. Activation can occur through the canonical or noncanonical pathways. Pro-inflammatory signaling leads to the activation of the TAK1 signalosome. TAK1 in turn activates IKK $\beta$, leading to the phosphorylation and degradation of IкBs. This leads to the nuclear translocation of p50/65 dimer. The noncanonical pathway involves the activation of NIK which causes the phosphorylation of IKK $\alpha$. Subsequently, IKK $\alpha$ causes the phosphorylation and proteolytic processing of p100, generating p52 protein which then make a complex with RelB and translocate to the nucleus to induce gene expression of specific molecules. . 


\section{CHAPTER 2}

\section{SATELLITE CELL-SPECIFIC ABLATION OF IKK $\beta$ RESULTS IN AN IMPAIRED REGENERATIVE PROCESS}

2.1 Introduction. Skeletal muscle is composed of multinucleated myofibers, which themselves arise from the fusion of myoblasts during embryonic development [122]. Although adult skeletal muscle is a post-mitotic tissue, it has a remarkable ability to regenerate in response to traumatic injury or strenuous exercise. Skeletal muscle regeneration is mediated by a subset of adult stem cells, termed satellite cells, which are located between the basal lamina and the sarcolemma in a relatively dormant state [122, 123]. Upon muscle injury, satellite cells enter the cell cycle, undergo several rounds of cell division, and then differentiate into myoblasts, which ultimately fuse with each other or with injured myofibers to complete the repair process. While a vast majority of satellite cells commit to the myogenic lineage, a fraction of them self-renews and returns to quiescence to replenish the satellite cell pool [124]. Satellite cells express the transcription factor paired box 7 (Pax7), which is essential for their self-renewal, proliferation, and maintenance of myogenic potential in adult skeletal muscle. The role of Pax7 in satellite cell homeostasis is evidenced by the findings that the targeted ablation of Pax7 results in the depletion of the satellite cell pool and impairment of skeletal muscle regeneration after traumatic injury $[125,126]$. 
Nuclear factor- $\mathrm{kB}(\mathrm{NF}-\mathrm{\kappa B})$ is a family of transcription factors, which regulate a large number of genes involved in survival, proliferation, and differentiation of both immune and non-immune cell types [127]. The NF- $\mathrm{BB}$ family contains five members: RelA (also known as p65), RelB, c-Rel, p105/p50, and p100/p52, which make homo- and heterodimers. In unstimulated cells, NF-kB proteins are normally sequestered in the

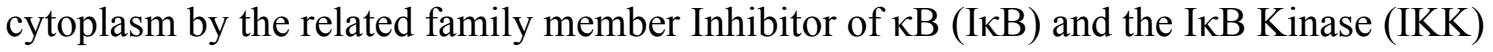
complex [128]. NF- $\mathrm{kB}$ can be activated through either the canonical or non-canonical pathway [127]. The canonical NF- $\mathrm{kB}$ signaling pathway involves the upstream activation of IKK $\beta$ and subsequent phosphorylation and degradation of IKB proteins, resulting in rapid and transient nuclear translocation of canonical NF- $\mathrm{kB}$ members, predominantly the p50/RelA and p50/c-Rel dimers. In the non-canonical pathway, a central signaling molecule is NF-kB-inducing kinase (NIK), which activates and cooperates with IKK $\alpha$ to mediate p100 phosphorylation, which in turn leads to p100 ubiquitination and degradation of its C-terminal IкB-like structure, resulting in the generation of mature NF-

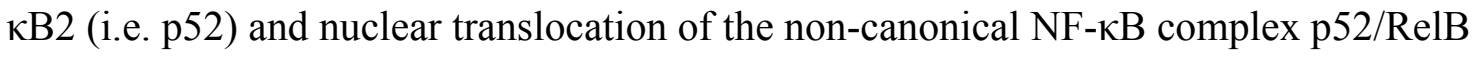
[128]. Published reports suggest that the activation of NF- $\mathrm{KB}$ reduces skeletal muscle mass, metabolic function, and regeneration especially in disuse conditions and chronic disease states [129-131]. Intriguingly, a recent study has demonstrated that sustained inhibition of NF-кB in myofibers increases the age-associated loss of skeletal muscle mass suggesting that physiological levels of NF- $\mathrm{KB}$ are essential to maintaining skeletal muscle health [132].

Studies using cultured myoblasts have suggested that the canonical and noncanonical NF- $\mathrm{KB}$ pathways play distinct roles in the regulation of myogenesis [133]. The 


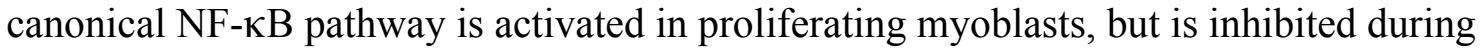
the differentiation of myoblasts into multinucleated myotubes [133]. Indeed, several proinflammatory cytokines inhibit myogenic differentiation through the activation of

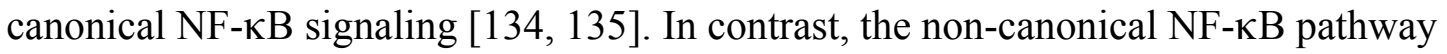
becomes activated during myogenic differentiation and promotes mitochondrial

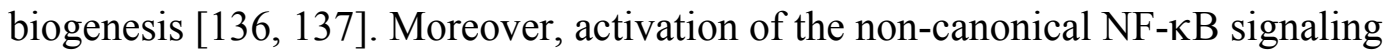
pathway promotes myoblast fusion during myogenesis [138, 139].

While the role of IKK $\beta$-mediated canonical NF-KB signaling in the regulation of myogenesis is evidenced, it remains enigmatic whether this pathway has any role in the regulation of satellite stem cell function. It has been reported that the activation of IKK $\beta$ in satellite cells is a reason for their reduced differentiation into the myogenic lineage in mouse models of cancer cachexia [116]. In contrast, we found that the proinflammatory cytokine, TWEAK, activates NF- $\mathrm{BB}$ in satellite cells. Interestingly, inhibition of canonical NF- $\mathrm{kB}$ signaling improves the number of Pax $7^{+}$cells in TWEAK-treated cultures suggesting that depending on the stimuli, the activation of NF- $\mathrm{KB}$ can differentially regulate the fate of satellite cells [140]. Recent studies from our group have shown that TAK1 and TRAF6, the upstream regulators of the canonical NF- $\mathrm{KB}$ signaling pathway, are essential for the survival, self-renewal, and proliferation of satellite cells in skeletal muscle of adult mice. Targeted ablation of either TAK1 or TRAF6 in satellite cells severely impairs skeletal muscle regeneration in adult mice $[65,117]$. However, the role of canonical NF- $\mathrm{kB}$ signaling in the regulation of satellite cell function during regenerative myogenesis has not yet been investigated. 
In this study, through the generation of an inducible satellite cell-specific IKK $\beta$ knockout (KO) mouse, we demonstrate that the canonical NF-KB signaling pathway in satellite cells is essential for the proper regeneration of skeletal muscle after injury. Our results also suggest that the canonical NF- $\mathrm{KB}$ pathway promotes survival and proliferation of satellite cells. In addition, we demonstrate that canonical NF- $\mathrm{B}$ signaling is essential to prevent precocious differentiation of activated satellite cells.

\subsection{Materials and methods.}

Animals. Satellite cell-specific inducible IKK $\beta$-knockout mice (i.e. P7:IKK $\beta$-KO) were

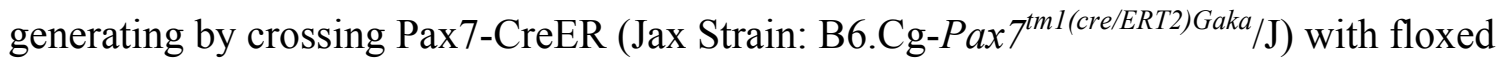
$\operatorname{IKK} \beta$ (i.e. IKK $\beta^{\mathrm{f} / \mathrm{f}}$ ) mice. All mice were in the C57BL/6J background and their genotype was determined by PCR from tail DNA. For Cre-mediated inducible deletion of IKK $\beta$, 6week and 12-week old mice were injected intraperitoneally (i.p.) with Tamoxifen (10 mg per Kg body weight) for five consecutive days. Control mice were injected with corn oil only. The Institutional Animal Care and Use Committee (IACUC) and Institutional Biosafety Committee (IBC) of the University of Louisville approved all experimental protocols with mice in advance.

Skeletal muscle injury. One week after the first injection of tamoxifen, $100 \mu \mathrm{L}$ of $1.2 \%$ Barium Chloride $\left(\mathrm{BaCl}_{2}\right.$, Sigma Chemical Co.) in saline was injected into the tibialis anterior (TA) muscle of mice to induce necrotic muscle injury. At various time points, TA muscle was collected from euthanized mice for biochemical and histological studies. 
Myoblast fusion. To study myoblast fusion, two days following $\mathrm{BaCl}_{2}$-mediated necrotic muscle injury in the TA, mice were given an i.p. injection of 5-ethnyl-2'-deoxyuridine (EdU; $4 \mu \mathrm{g}$ per gm body weight). Twelve days post-EdU injection, the TA was isolated and transverse muscle sections made. The sections were subsequently immunostained with anti-Laminin, DAPI for the detection of nuclei, and processed for the detection of EdU. The number of interstitial and intramyofibrillar EdU ${ }^{+}$myonuclei per myofiber was quantified using NIH ImageJ software.

Histology and morphometric analysis. For skeletal muscle morphology and regeneration assessment, $10 \mu \mathrm{m}$-thick transverse sections of the TA were stained with Hematoxylin and Eosin. For quantitative analysis, CSA, minimum Feret's diameter, and multinucleation were analyzed in H\&E stained TA muscle sections. H\&E slides were mounted using DPX Mountant For Histology Slide mounting medium (Sigma Chemical Co.) and visualized at room temperature on a Nikon Eclipse TE 2000-U Microscope (Nikon), a digital camera (Nikon Digital Sight DS-Fi1), and Nikon NIS Elements BR 3.00 software (Nikon). Exposure time was kept at $80 \mathrm{~ms}$ and contrast levels were not altered.

Satellite cell cultures. Satellite cells were isolated from the hind limbs of 6- to 8-weekold C57BL/6J mice as described [117]. For siRNA experiments, cells were transfected using the RNAiMAX Lipofectamine system using a protocol from the manufacturer. Control siRNA-A (cat\# sc-37007), mouse NF-kB p65 siRNA (cat\# sc-29411), and mouse IKK beta siRNA (cat\# sc-35645) were obtained from Santa Cruz Biotechnology. For 
overexpression plasmid studies, cells were transfected using the Neon Electroporation transfection system using a protocol from the manufacturer with settings of: $1500 \mathrm{~V}$, 10ms pulse width, for three pulses. pCMV4 p65 was a gift from Warner Greene (Addgene plasmid \# 21966) [141]. pcDNA-Ikkb-FLAG WT was a gift from Warner Greene (Addgene plasmid \# 23298) [142]. pcDNA3.1 (Invitrogen) was used as a control for the pCMV4 p65 and pcDNA-Ikkb-FLAG WT plasmids.

Isolation and culturing of myofibers. Single myofiber cultures were established from extensor digitorum longus (EDL) muscle after digestion with collagenase II (Worthington Biochemical Corporation, Lakewood, NJ) and trituration as previously described [117]. Suspended myofibers were collected immediately or cultured in $60 \mathrm{~mm}$ horse serumcoated plates in Dulbecco's modified Eagle's medium (DMEM) supplemented with: 10\% fetal bovine serum (FBS; Invitrogen), 2\% chicken embryo extract (Accurate Chemical, Westbury, NY), $10 \mathrm{ng} / \mathrm{mL}$ basic fibroblast growth factor (Peprotech, Rocky Hill, NJ), and $1 \%$ penicillin-streptomycin for three days.

Immunofluorescence. For IHC and ICC studies, frozen TA muscle sections (9 or $10 \mu \mathrm{m}-$ thick sections) or myoblast/myofiber cultures, respectively, were fixed in $4 \%$ paraformaldehyde (PFA) in phosphate buffered saline (PBS), blocked in 2\% bovine serum albumin (BSA) in PBS for one hour, and subsequently incubated with anti-Pax7 (1:5-1:10, DSHB Cat\# pax7, RRID:AB_528428), anti-eMyHC (1:200, DSHB Cat\# F1.652, RRID:AB_447163), anti-MyoD (1:200, Santa Cruz Biotechnology Cat\# sc-304, RRID:AB_631992), anti-Laminin (1:150, Cell Signaling Technology Cat\# L9393, 
RRID:AB_477163), or anti-p-p65 (1:100, Cell Signaling Technology Cat\# 3033, RRID:AB_331284) in blocking solution at $4^{\circ} \mathrm{C}$ overnight under humidified conditions. The sections were washed briefly with PBS before incubation with its respective secondary antibody for one hour at room temperature and then washed three times for five minutes with PBS. Nuclei were visualized by counterstaining with DAPI for five minutes. Refer to Appendix-3 for complete antibody listing.

EdU and TUNEL Staining. To determine the proliferation status of satellite cells, EdU staining was performed using a commercially available kit and following the protocol from the manufacturer (Click-iT EdU Cell Proliferation Assay kit, Invitrogen). Briefly, during the last 90 minutes of incubation, $10 \mu \mathrm{M}$ EdU (Invitrogen) was added in culture medium. The cells were fixed in 4\% PFA. EdU visualization occurs through a click reaction, whereby the EdU is covalently bonded to a fluorescent label (Alexa Fluor ${ }^{\circledR} 488$ Azide). Nuclei were visualized by counterstaining with DAPI for 5 minutes. TUNEL staining was performed following a protocol from the manufacturer (in situ Cell Death Detection Kit, Sigma Aldrich). Briefly, the sections or myofiber cultures were fixed in $4 \%$ PFA and permeabilized with $0.1 \%$ Triton $\mathrm{X}-100$ in $0.1 \%$ sodium citrate, and then incubated in TUNEL reaction mixture for 60 minutes at $37^{\circ} \mathrm{C}$.

Fluorescent Imaging. ICC/IHC slides were mounted using Aqua-Poly/Mount fluorescence medium (Polysciences, Inc) and visualized at $-0.4^{\circ} \mathrm{C}$ on a Nikon TiE 3000 Inverted Microscope (Nikon), a digital camera (DXM-1200C Coded Digital Camera), and Nikon NIS Elements AR software (Nikon). Image levels were equally adjusted using 
Adobe Photoshop CS6 software (Adobe). Detailed specifications, exposure times, and contrast levels provided in Appendix-4.

Western blot. Relative levels of various proteins were quantified by performing Western blot as described [65]. Briefly, skeletal muscle of mice or cultured myoblasts was washed with PBS, homogenized in lysis buffer (50 mM Tris-Cl (pH 8.0), $200 \mathrm{mM} \mathrm{NaCl}, 50 \mathrm{mM}$ NaF, $1 \mathrm{mM}$ dithiothreitol, $1 \mathrm{mM}$ sodium orthovanadate, 0.3\% IGEPAL, and Protease Inhibitor Cocktail). Approximately, $100 \mu \mathrm{g}$ of protein was resolved on each lane on a $10 \%$ SDS-PAGE, electro-transferred onto a nitrocellulose membrane and probed using various primary antibodies. Detection of proteins was enhanced by chemiluminescence; refer to Appendix-3 for list of proteins. As a loading control, membranes were stripped and re-probed with anti-GAPDH (1:2000, Cell Signaling Technology Cat\# 2218, RRID:AB_561053).

RNA isolation and quantitative Real-time PCR (qRT-PCR) Assay. RNA isolation and qRT-PCR were performed as previously described [117]. Total RNA was extracted from skeletal muscle tissue of mice or cultured myogenic cells using the TRIzol reagent (Thermo Fisher Scientific Life Sciences) and an RNeasy Mini Kit (Qiagen, Valencia, CA, USA) according to the manufacturers' protocols. First-strand cDNA for PCR analyses were generated with a commercially available kit (Thermo Fisher Scientific Life Sciences). Quantification of mRNA expression was performed using the SYBR Green dye (Thermo Fisher Scientific Life Sciences) method on a sequence-detection system (model 7300; Thermo Fisher Scientific Life Sciences). Approximately $25 \mu$ of reaction 
volume was used for the real-time PCR assays which consisted of $2 \times(12.5 \mu \mathrm{l})$ Brilliant SYBR Green QPCR Master Mix (Stratagene), $400 \mathrm{~nm}$ of primers ( $0.5 \mu$ l each from the stock), $11 \mu \mathrm{l}$ of water, and $0.5 \mu \mathrm{l}$ of template. The thermal conditions consisted of an initial denaturation at $95{ }^{\circ} \mathrm{C}$ for 10 min followed by 40 cycles of denaturation at $95{ }^{\circ} \mathrm{C}$ for $15 \mathrm{~s}$, annealing and extension at $60^{\circ} \mathrm{C}$ for $1 \mathrm{~min}$, and, for a final step, a melting curve of $95^{\circ} \mathrm{C}$ for $15 \mathrm{~s}, 60^{\circ} \mathrm{C}$ for $15 \mathrm{~s}$, and $95^{\circ} \mathrm{C}$ for $15 \mathrm{~s}$. All reactions were carried out in triplicate to reduce variation. Primers were designed using Vector NTI software (Thermo Fisher Scientific Life Sciences). The sequences of the primers used are as follows: $\boldsymbol{\beta}$ actin, 5'-CAG GCA TTG CTG ACA GGA TG-3' (forward) and 5'-TGC TGA TCC ACA TCT GCT GG-3' (reverse); IF $\boldsymbol{N}-\boldsymbol{\gamma}$, 5' $^{\prime}$ GAC AAT CAG GCC ATC AGC AAC-3' (Forward), 5'-CGG ATG AGC TCA TTG AAT GCT T-3'; $\boldsymbol{I} \boldsymbol{L}-\mathbf{1} \boldsymbol{\beta}$, 5'-CTC CAT GAG CTT TGT ACA AGG-3' (Forward), 5'-TGC TGA TGT ACC AGT TGG GG-3' (Reverse); IL-6, 5'-ATG GCA ATT CTG ATT GTA TG -3' (Forward), 5'-TGG CTT

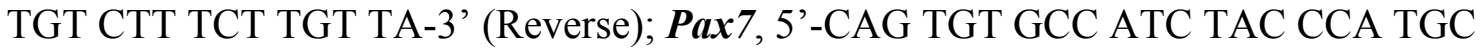
TTA-3' (Forward), 5'-GGT GCT TGG TTC AAA TTG AGC C-3' (Reverse); $\boldsymbol{M y f 5}$, 5'TGA AGG ATG GAC ATG ACG GAC G-3' (Forward), 5'-TTG TGT GCT CCG AAG GCT GCT A-3' (Reverse); $\boldsymbol{M y} \boldsymbol{h} 3$, 5'-ACA TCT CTA TGC CAC CTT CGC TAC-3' (Forward), 5'-GGG TCT TGG TTT CGT TGG GTA T-3' (Reverse); $\boldsymbol{M y} \boldsymbol{h} 4$, 5'-CGG CAA TGA GTA CGT CAC CAA A-3' (Forward), 5'-TCA AAG CCA GCG ATG TCC AA-3' (Reverse); MyoD, 5'-TGG GAT ATG GAG CTT CTA TCG C-3' (Forward), 5'GGT GAG TCG AAA CAC GGA TCA T-3' (Reverse); Myogenin, 5'-CAT CCA GTA CAT TGA GCG CCT A-3' (Forward), 5'-GAG CAA ATG ATC TCC TGG GTT G-3'

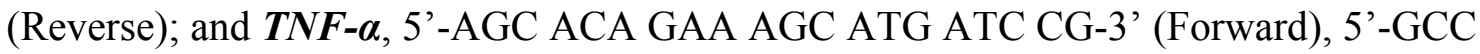


ACA AGC AGG AAT GAG AA-3' (Reverse). The data were analyzed using SDS software version 2.0, and the results were exported to Microsoft Excel for further analysis. Data normalization was accomplished using two endogenous control ( $\beta$-actin) and the normalized values were subjected to a $2^{-\Delta \Delta \mathrm{Ct}}$ formula to calculate the fold change between the control and experimental groups. The formula and its derivations were obtained from the ABI Prism 7900 Sequence Detection System user guide.

Lactate dehydrogenase (LDH) assay. The amount of LDH in culture supernatants was measured using a commercially available LDH Cytotoxicity Assay kit following the protocol suggested by the manufacturer (Thermo Scientific Life Sciences).

Statistical analyses. For the sake of transparency, results were expressed as box-andwhisker plots with the box comprised of the $1^{\text {st }}, 2^{\text {nd }}$, and $3^{\text {rd }}$ quartiles, and the lower and upper whiskers corresponding to the minimum and maximum values, respectively, to display the entire range of data. Statistical analyses between two groups used unpaired two-tailed Student's t-test to compare quantitative data populations with normal distribution and equal variance. A value of $p<0.05$ was considered statistically significant unless specified otherwise for comparisons made between two groups.

\subsection{Results}

\subsubsection{Canonical NF-кB signaling is activated in satellite cells after skeletal muscle} injury. We first investigated how the levels of different components of canonical NF-кB pathway are regulated in skeletal muscle upon injury. Tibialis anterior (TA) muscle of 
12-week old wild-type (WT) mice was injured by intramuscular injection of $1.2 \% \mathrm{BaCl}_{2}$ solution, whereas the contralateral muscle was injected with saline alone and served as an uninjured control. At day 5 after injury, the muscle was collected and analyzed by performing immunoblotting or immunohistochemical analysis. There was a noticeable increase in the levels of IKK $\beta$ in 5d-injured TA muscle compared to contralateral uninjured muscle. Moreover, the levels of phosphorylated, as well as total, $\mathrm{I} \kappa \mathrm{B} \alpha$ and $\mathrm{p} 65$ proteins were considerably increased in injured muscle compared to uninjured controls (Figure 2.1A). To understand whether muscle injury leads to the activation of canonical NF-кB signaling in satellite cells, we performed double immunostaining for phospho-p65 (p-p65) and Pax7 (a marker for satellite cells) proteins. Consistent with a published report [143], p-p65 protein was undetectable in satellite cells of uninjured muscle, however, the expression of p-p65 protein was clearly visible in $\mathrm{Pax} 7^{+}$cells in injured muscle (Figure 2.1B).

A suspension culture of myofiber explants represents an ex vivo model that mimics muscle injury in vivo, with respect to satellite cell survival, activation, proliferation, and differentiation $[117,144,145]$. To further understand whether canonical NF- $\mathrm{KB}$ signaling is activated in satellite cells upon injury, we established single myofiber cultures from extensor digitorum longus (EDL) muscle of WT mice and myofiber-associated satellite cells were examined for the expression of p-p65 protein at different time points. We could not detect p-p65 protein in satellite cells (i.e. Pax $7^{+}$) just after isolation of myofibers (Figure 2.1C). Interestingly, a gradual increase in p-p65 levels in $\mathrm{Pax}^{+}$cells was evidenced at $24 \mathrm{~h}$ and $72 \mathrm{~h}$ after establishing the cultures (Figure 
2.1C). These results suggest that muscle injury leads to the activation of canonical NF$\kappa \mathrm{B}$ signaling in satellite cells of adult mice.

\subsubsection{Satellite cell-specific deletion of IKK $\beta$ delays myofiber regeneration in adult}

mice. In response to various cytokines, growth factors, and microbial products, the activation of the canonical NF-אB pathway involves the stimulation of IKK $\beta$ [127]. To understand the role of canonical NF- $\mathrm{KB}$ signaling in the regulation of satellite cell homeostasis and function, we crossed floxed $\operatorname{IKK} \beta\left(\operatorname{IKK} \beta^{\mathrm{f} / \mathrm{f}}\right)$ mice that are homozygous for loxP sites flanking Exon 3 with Pax7-CreER mice (a tamoxifen-inducible satellite cell-specific Cre line) [146] to generate satellite cell-specific inducible IKK $\beta$-knockout $\left(\mathrm{IKK} \beta^{\mathrm{fff}}\right.$; Pax7-CreER) mice (Figure 2.2A). Since Pax7-CreER mice are knock-in mice in which the expression of $\operatorname{Pax} 7$ is regulated by the endogenous Pax 7 promoter, we used adult IKK $\beta^{\text {fff }}$;Pax7-CreER mice and treated them with tamoxifen or vehicle (corn oil) alone to generate satellite cell-specific IKK $\beta$ knockout (henceforth P7:IKK $\beta$-KO) or control (Ctrl) mice, respectively. The mice were subjected to five intraperitoneal injections of tamoxifen or vehicle (Figure 2.2B). One week after the first injection of tamoxifen, TA muscle of Ctrl and P7:IKK $\beta$-KO mice was injected with $100 \mu$ of $1.2 \%$ $\mathrm{BaCl}_{2}$ solution unilaterally to induce necrotic muscle injury, whereas the contralateral muscle was injected with saline and served as an uninjured control. The uninjured and injured TA muscles were isolated at $5 \mathrm{~d}$ and $14 \mathrm{~d}$ post-injury and analyzed by performing Hematoxylin and Eosin (H\&E) staining (Figure 2.2C). Under naïve conditions, we found no statistically significant difference in the average cross-sectional area (CSA) or minimal Feret's diameter between P7:IKK $\beta$-KO and littermate Ctrl mice (data not 
shown). Interestingly, we found that the regeneration of TA muscle was considerably diminished in $\mathrm{P}$ 7:IKK $\beta$-KO mice compared to littermate $\mathrm{Ctrl}$ mice at $5 \mathrm{~d}$ post $\mathrm{BaCl}_{2}$ mediated injury. Morphometric analysis of 5d-injured TA muscle sections showed an approximate $40 \%$ reduction in average myofiber CSA in $\mathrm{P} 7: \mathrm{IKK} \beta-\mathrm{KO}$ mice compared to littermate Ctrl mice (Figure 2.2D). Additionally, a significant reduction in the average minimal Feret's diameter of myofibers was noticeable in 5d-indured TA muscle of P7:IKK $\beta$-KO mice compared to littermate Ctrl mice (Figure 2.2E). Newly formed, centrally multinucleated myofibers populated the regenerating TA muscle of $\mathrm{Ctrl}$ mice. However, the P7:IKK $\beta$-KO mice showed a marked decrease in centrally multinucleated myofibers compared to littermate $\mathrm{Ctrl}$ mice, indicating further signs of an impaired regeneration (Figure 2.2F). Although muscle structure appeared comparable, a significant reduction in average myofiber CSA and a trend towards reduction in minimal Feret's diameter $(\mathrm{p}=0.08)$ was noticeable in TA muscle of P7:IKK $\beta-\mathrm{KO}$ mice compared to littermate Ctrl mice even after 14d of injury (Figure 2.2G, 2.2H). For some of our initial experiments, 12-week old littermate IKK ${ }^{\mathrm{f} / \mathrm{f}}$ and IKK $\beta^{\mathrm{f} / \mathrm{f}}$; Pax7-CreER mice were treated by i.p. injections of tamoxifen similar to as described above (Figure 2.2B). TA muscle of these mice was injured and muscle regeneration was studied at day 5 postinjury by performing H\&E staining (data not shown). Similar to the above results, we found that the muscle regeneration was reduced in IKK $\beta^{\mathrm{fff}}$; Pax7-CreER mice compared to IKK $\beta^{\mathrm{f} / \mathrm{f}}$ mice suggesting that the deletion of IKK $\beta$ in satellite cells reduces the regeneration of skeletal muscle, whereas the treatment of tamoxifen itself does not (data not shown). 


\subsubsection{Targeted inactivation of IKK $\beta$ diminishes the number of nascent myofibers}

and satellite cells in regenerating muscle of adult mice. To further confirm that the deletion of IKK $\beta$ in satellite cells reduces myofiber regeneration, we also examined the number of myofibers expressing the embryonic isoform of myosin heavy chain (eMyHC). Consistent with the notion that eMyHC is expressed in newly found myofibers, we did not find any eMyHC ${ }^{+}$myofibers in uninjured TA muscle of $\mathrm{Ctrl}$ or $\mathrm{P7}$ :IKK $\beta-\mathrm{KO}$ mice. By contrast, the number of $\mathrm{eMyHC}^{+}$myofibers was dramatically increased in 5d-injured TA muscle. However, the frequency of eMyHC ${ }^{+}$myofibers within laminin staining (Figure 2.3B) and the size of eMyHC ${ }^{+}$myofibers (Figure 2.3C, 2.3D) were significantly reduced in TA muscle of $P 7: I K K \beta-K O$ mice compared with corresponding Ctrl mice.

We next investigated the effect of deletion of IKK $\beta$ on satellite cell number. TA muscle of Ctrl and P7:IKK $\beta$-KO mice were immunostained for Pax7 and Laminin proteins. Nuclei were labeled by staining with DAPI (Figure 2.3E). There was no significant difference in the number of $\operatorname{Pax} 7^{+}$positive cells in uninjured TA muscle of Ctrl and P7:IKK $\beta$-KO mice (data not shown). Interestingly, we found that the number of $\mathrm{Pax}^{+}$cells was significantly reduced in 5d-injured TA muscle of P7:IKK $\beta$-KO mice compared to littermate $\mathrm{Ctrl}$ mice (Figure 2.3E, 2.3F). Furthermore, mRNA levels of Pax 7 were found to be significantly reduced in 5d-injured TA muscle of P7:IKK $\beta$-KO mice compared to littermate Ctrl mice (Figure 2.3G). These results suggest that the deletion of IKK $\beta$ reduces satellite cell number following injury, which may be responsible for the delayed myofiber regeneration. 
Activation of canonical NF- $\mathrm{BB}$ signaling leads to the expression of a number of inflammatory cytokines $[127,128]$. However, it remains unknown whether satellite cells are also the source of inflammatory molecules in injured skeletal muscle. Nevertheless, by performing qRT-PCR, we measured relative mRNA levels of proinflammatory cytokines: interleukin (IL)-1 $\beta, I L-6$, and tumor necrosis factor- $\alpha(T N F-\alpha)$. There was no significant difference in the mRNA levels of these cytokines in 5d-injured TA muscle of Ctrl and P7:IKK $\beta-$ KO mice (Figure 2.3H), suggesting that the deletion of IKK $\beta$ in satellite cells does not affect the expression of inflammatory cytokines.

\subsubsection{Inhibition of canonical NF- $\kappa$ B signaling reduces the proliferation of satellite}

cells. To understand cellular mechanisms for the reduced number of satellite cells in regenerating skeletal muscle of $\mathrm{P} 7: \mathrm{IKK} \beta-\mathrm{KO}$ mice, we investigated the role of canonical $\mathrm{NF}-\kappa \mathrm{B}$ signaling in the proliferation of satellite cells in vivo, ex vivo, and in vitro. TA muscle of 12-week old Ctrl and P7:IKK $\beta$-KO mice was injured by intramuscular injection of $1.2 \% \mathrm{BaCl}_{2}$ solution. After $48 \mathrm{~h}$, the mice were given an intraperitoneal injection of EdU and the number of $\mathrm{EdU}^{+}$nuclei in the TA muscle was determined 12 days later. Interestingly, a significant reduction in both intramyofibrillar and interstitial $\mathrm{EdU}^{+}$nuclei was observed in TA muscle of P7:IKK $\beta-\mathrm{KO}$ mice compared with corresponding $\mathrm{Ctrl}$ mice (Figure 2.4A, 2.4B). To further understand whether IKK $\beta$ regulates the proliferation of satellite cells, we established myofiber explant cultures and the proliferation of myofiber-associated cells were studied by measuring EdU incorporation after $72 \mathrm{~h}$ of culturing. Consistent with our in vivo results, we found that the 
proportion of $\mathrm{EdU}^{+}$nuclei was significantly reduced in myofiber explants prepared from P7:IKK $\beta$-KO mice compared to Ctrl mice (Figure 2.4C, 2.4D).

We also investigated the role of canonical NF-KB signaling in the proliferation of satellite cell-derived myogenic cultures in a myofiber-free system. For this experiment, primary myogenic cultures established from WT mice were transfected with scrambled (control), IKK $\beta$, or p65 siRNA. After 72h, cellular proliferation was studied by performing an EdU incorporation assay. Results showed that the knockdown of IKK $\beta$ and $\mathrm{p} 65$ significantly reduced the proportion of $\mathrm{EdU}^{+}$nuclei in myogenic cultures (Figure 2.4E, 2.4F). Our Western blot analysis also showed that the levels of cyclin D1 and cyclin A were significantly reduced in cultures transfected with IKK $\beta$ siRNA or p65 siRNA compared to control cultures (Figure 2.4G). Western blot analysis confirmed a drastic reduction in IKK $\beta$ and $p 65$ protein upon transfection with their corresponding siRNAs (Figure 2.4G).

\subsubsection{Canonical NF-KB signaling promotes the survival of satellite cells. One of the} important functions of the canonical arm of NF-KB signaling is to promote cell survival $[127,128]$. Our attempts to perform double immunostaining for TUNEL and Pax7 were futile, possibly because the levels of Pax7 are reduced in satellite cells undergoing apoptosis. Therefore, we employed ex vivo myofiber explants and cultured myogenic cells to understand the role of the canonical arm of NF- $\mathrm{kB}$ signaling in the survival of satellite cells. We first established myofiber explant cultures from EDL muscle of Ctrl and P7:IKK $\beta-K O$ mice. After 72h of culturing, the percentage of apoptotic cells was measured by performing TUNEL staining. Interestingly, the proportion of myofiber- 
associated $\mathrm{TUNEL}^{+}$cells was significantly higher in P7:IKK $\beta$-KO cultures compared to Ctrl cultures (Figure 2.5A, 2.5B).

We next investigated the effect of siRNA-mediated knockdown of IKK $\beta$ or p65 on survival of cultured primary myogenic cells. Lactate dehydrogenase (LDH) is a stable enzyme that accumulates in culture supernatants after cell death [147]. Interestingly, we found that the levels of LDH in culture supernatants of cells transfected with IKK $\beta$ and p65 siRNA were significantly higher compared to those transfected with control siRNA (Figure 2.5C). We also performed Annexin V/propidium iodide (PI) staining on these cells followed by analysis using FACS. Similar to our LDH results, we found that the percentages of early and late apoptotic cells were significantly increased in cultures transfected with IKK $\beta$ and p65 siRNA compared to those transfected with scrambled siRNA (Figure 2.5D-F). We also performed Western blotting to measure the levels of cleaved Caspase-3 and cleaved PARP, two established markers of apoptosis. As shown in Figure 2.5G, the levels of cleaved PARP were significantly increased in cultures transfected with IKK $\beta$ or p65 siRNA compared to those transfected with scrambled siRNA. Moreover, we found that the knockdown of IKK $\beta$ or p65 led to a reduction in levels of BAX and Bc12 protein in cultured myogenic cells (Figure 2.5G). Collectively, these results suggest that canonical NF- $\mathrm{KB}$ signaling promotes the survival of myogenic cells.

\subsubsection{Canonical NF- $\mathrm{KB}$ signaling promotes the self-renewal and prevents the} precocious differentiation of activated satellite cells. Pax 7 determines satellite cell fate in cooperation with other factors, such as MyoD [117, 144, 147]. Indeed, the expression 
pattern of Pax7 and MyoD specifies the myogenic status of satellite cells as quiescent $\left(\mathrm{Pax}^{+} / \mathrm{MyoD}^{-}\right)$, activated $\left(\mathrm{Pax}^{+} / \mathrm{MyoD}^{+}\right)$, or differentiated $\left(\mathrm{Pax}^{-} / \mathrm{MyoD}^{+}\right)$. We investigated whether IKK $\beta$ has any role in the regulation of satellite cell fate. To model the satellite cell dynamics of muscle injury, we generated ex vivo suspension cultures of EDL myofibers from P7:IKK $\beta-\mathrm{KO}$ and Ctrl mice. The ex vivo suspension cultures were analyzed by staining with anti-Pax 7 and anti-MyoD at $0 \mathrm{~h}$ or $72 \mathrm{~h}$ of culturing (Figure 2.6A, 2.6B). There was no significant difference in the number of myofiber-associated $\mathrm{Pax}^{+}$cells between $\mathrm{Ctrl}$ and P7:IKK $\beta-\mathrm{KO}$ cultures at $0 \mathrm{~h}$ (Figure 2.6A, 2.6C). There were a negligible number of myofiber-associated $\mathrm{MyoD}^{+}$cells and there was no significant difference in their number between $\mathrm{Ctrl}$ and P7:IKK $\beta$-KO cultures (Figure 2.6D). Myofiber-associated satellite cells formed clusters by $72 \mathrm{~h}$ of culturing. Although there was no significant difference in the average number of clusters per myofiber, the average number of cells per cluster was significantly reduced in P7:IKK $\beta$-KO cultures compared to Ctrl cultures (Figure 2.6E). Our analysis also showed that the proportion of $\mathrm{Pax}^{+} / \mathrm{MyoD}^{-}$, as well as $\mathrm{Pax}^{+} / \mathrm{MyoD}^{+}$, cells was significantly reduced in P7:IKK $\beta-\mathrm{KO}$ cultures compared to Ctrl cultures (Figure 2.6F, 2.6G). Conversely, the percentage of Pax7 $7 / \mathrm{MyoD}^{+}$was dramatically increased in P7:IKK $\beta-\mathrm{KO}$ compared with Ctrl cultures (Figure 2.6H), suggesting that the inactivation of IKK $\beta$ causes a precocious differentiation of activated satellite cells.

We also investigated the role of canonical NF-KB signaling in the regulation of satellite cell fate in a myofiber-free environment. We first established satellite cellderived primary myogenic cultures from hind limb muscles of WT mice. Early passage myogenic cells were transfected with scrambled (control), IKK $\beta$, or p65 siRNA. After 
72h, the cultures were fixed followed by staining with anti-Pax7 and anti-MyoD. Nuclei were labeled using DAPI (Figure 2.7A). Consistent with published reports [117, 147], we found that the majority of cells were $\mathrm{Pax}^{+} / \mathrm{MyoD}^{+}$, whereas a small proportion were $\mathrm{Pax}^{+} / \mathrm{MyoD}^{-}$in cultures transfected with control siRNA. Although there was a trend towards reduction in the percentage of $\mathrm{Pax}^{+} / \mathrm{MyoD}^{-}$cells in cultures transfected with IKK $\beta$ or p65 siRNA, it was not significantly different from those transfected with control siRNA (Figure 2.7B). However, the proportion of $\mathrm{Pax} 7^{+} / \mathrm{MyoD}^{+}$cells was significantly reduced, whereas the proportion of $\mathrm{Pax} 7^{-} / \mathrm{MyoD}^{+}$cells was significantly increased in IKK $\beta$ or p65 knocked-down cultures compared to control cultures (Figure 2.7C, 2.7D). By performing immunostaining for myosin heavy chain (MyHC) protein, we also investigated whether the knockdown of IKK $\beta$ or p65 can induce the terminal differentiation of satellite cell-derived myoblasts under growth conditions. There was a negligible number of $\mathrm{MyHC}^{+}$cells in myogenic cultures transfected with scrambled or IKK $\beta$ siRNA (Figure 2.7E). Intriguingly, we found that the knockdown of p65 increased the number of $\mathrm{MyHC}^{+}$cells. Consistent with our immunocytochemistry results, immunoblotting showed that the levels of Pax 7 were somewhat reduced, whereas levels of myogenin and $\mathrm{MyHC}$ were increased especially in cultures transfected with p65 siRNA (Figure 2.7F).

2.4 Discussion. Satellite cells normally exist in a quiescent state [123], but are rapidly activated following injury to skeletal muscle [35]. Similar to other stem cells, satellite cells possess the ability to self-renew and maintain a reserve pool in healthy tissue, such that they can facilitate several episodes of regeneration $[17,33,35]$. Diminishing the 
satellite cell pool is associated with an impairment in regenerative myogenesis and can lead to muscle wasting normally observed in genetic muscle disorders and during aging [148-150]. NF-kB plays a critical role in the regulation of cell fate, survival, and differentiation in both immune and non-immune cell types [127, 151]. Activation of NFКB also leads to an inflammatory response, especially in chronic disease states [127, 128]. While components of the canonical NF- $\mathrm{KB}$ pathway have been found to be activated in myofibers in various catabolic states, as well as in both myogenic and inflammatory immune cells in muscle degenerative disorders [113, 129, 152], the physiological role of NF- $\mathrm{KB}$ in the regulation of satellite cell function remains less understood. Our results in the present study demonstrate that canonical NF- $\mathrm{KB}$ signaling mediated by IKK $\beta$ and $\mathrm{p} 65$ promotes the survival, proliferation, and self-renewal of satellite cells.

Skeletal muscle regeneration is a complex process that is regulated by signals released from both damaged myofibers, as well as several other cell types that are either resident in the muscle or recruited to assist in clearing cellular debris $[122,123]$. Activation of satellite cells upon muscle injury requires transcriptional and translational reprogramming that is essential for their exit from quiescence, proliferation, and survival in a metabolically activate state [124, 153-155]. Our results demonstrate that the activation of the canonical arm of the NF-KB pathways is increased in the satellite cells upon skeletal muscle injury (Figure 2.1). This activation of NF-кB appears to be a mechanism to improve the proliferation and survival of satellite cells, evidenced by the finding that both the proliferation (Figure 2.4) and survival (Figure 2.5) of satellite cells is diminished upon genetic or siRNA-mediated knockdown of the components of the 


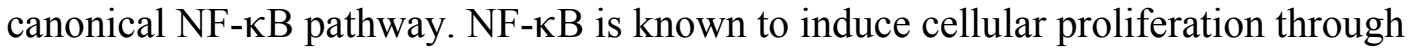
augmenting the expression of a number of growth regulatory molecules and cell cycle

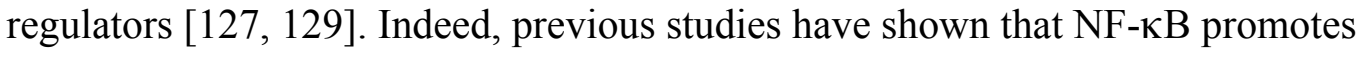
myogenic cell proliferation through increasing the expression of cyclin D1 [156]. Similarly, NF- $\mathrm{kB}$ is known to induce the expression of several anti-apoptotic molecules, such as Bcl2 [127, 128]. Our results demonstrate that the levels of both Cyclin D1 and Bcl2 were diminished upon knockdown of either IKK $\beta$ or p65 in cultured satellite cells (Figure 2.4G, 2.5G). Reduced proliferation and/or increased cell death through apoptosis appear to be important mechanisms for the reduced number of satellite cells observed in regenerating skeletal muscle of P7:IKK $\beta$-KO mice.

For efficient regeneration of skeletal muscle, satellite cells have to undergo extensive proliferation before their differentiation and fusion with injured myofibers. Premature differentiation or defects in the process of differentiation of satellite cells into the myogenic lineage can lead to deficits in skeletal muscle regeneration. Although the molecular mechanisms remain unknown, recent studies have suggested that NF- $\mathrm{KB}$ may play a role in the regulation of satellite cell self-renewal and differentiation. It has been reported that in the "settings" of cancer cachexia, the ability of satellite cells to differentiate into the myogenic lineage is lost, which may be a mechanism for cancerinduced muscle wasting [116]. Interestingly, the activation of the canonical arm of NF$\kappa \mathrm{B}$ signaling has been found to be one of the reasons for deregulation of Pax7 expression and satellite cell dysfunction in tumor-bearing mice [116]. However, the role of canonical NF- $\mathrm{BB}$ signaling in the self-renewal of satellite cell in naïve conditions or during regenerative myogenesis remains unknown. 
We found no significant difference in the number of quiescent/self-renewing $\left(\mathrm{Pax}^{+} / \mathrm{MyoD}^{-}\right)$satellite cells in skeletal muscle of $\mathrm{Ctrl}$ and IKK $\beta-\mathrm{KO}$ mice in uninjured muscle, suggesting that the inhibition of canonical NF- $\mathrm{KB}$ signaling does not disrupt satellite cell homeostasis in naïve conditions. Interestingly, our results demonstrate that the inhibition of canonical NF- $\mathrm{kB}$ signaling reduces the proliferation of activated satellite cells and causes their premature differentiation (Figures 2.6 and 2.7). While previous studies have shown that constitutive activation of canonical NF- $\mathrm{KB}$ signaling inhibits myogenic differentiation $[129,133]$, the results of the present study suggest that NF-אB signaling is essential to support the proliferation of activated satellite cells. It is important to note that while knockdown of IKK $\beta$ reduced the number of proliferating $\left(\mathrm{Pax}^{+} / \mathrm{MyoD}^{+}\right)$and increased the number of differentiating $\left(\mathrm{Pax}^{-} / \mathrm{MyoD}^{+}\right)$cells, it was not sufficient to induce terminal differentiation of satellite cells. By contrast, knockdown of p65 also resulted in the expression of MyHC, a marker of terminal differentiation of myogenic cells. This could be attributed to the fact that in addition to IKK $\beta$, the p65 subunit of NF- $\mathrm{\kappa B}$ can also be activated through signaling cross-talk and post-translational modifications [127]. Moreover, since p65 is the main subunit of the canonical NF- $\mathrm{KB}$ complex with transactivation domain, its knockdown may lead to a complete blockade of canonical NF-KB signaling. Indeed, our experiments demonstrate that compared to IKK $\beta$, knockdown of p65 has more pronounced effects on proliferation, differentiation, and survival of cultured satellite cells (Figures 2.4, 2.5, and 2.7). On similar lines, a previous study has demonstrated that muscle-derived stem cells (MDSCs) from $\mathrm{p} 65^{+/-}$mice show increased differentiation potential compared to those isolated from littermate WT mice [157]. 
The role of NF- $\mathrm{KB}$ in skeletal muscle regeneration has been previously investigated using genetic mouse models and molecular and pharmacological approaches $[129,158]$. NF- $\mathrm{kB}$ is highly activated in dystrophic muscle of $M d x$ (a mouse model of DMD) mice $[113,159]$. Interestingly, the genetic ablation of IKK $\beta$ in myofibers or in macrophages improved myopathy observed in $M d x$ mice [113]. Similarly, pharmacological inhibition of NF-кB using NEMO binding domain peptide was effective in improving muscle pathogenesis in $M d x$ mice $[113,160]$. Another study showed that the genetic ablation of IKK $\beta$ in myofibers improves skeletal muscle strength and regeneration, and inhibits the accumulation of fibrosis in otherwise normal mice [131]. On similar lines, we have demonstrated that the inhibition of TRAF6 in myofibers improves skeletal muscle regeneration potentially through inhibition of NF-kB [144]. However, all of these studies were performed using mice in which the components of canonical NF- $\kappa \mathrm{B}$ signaling were inhibited in myofibers and the improvement in muscle regeneration was attributed to a reduction in the inflammatory milieu in the injured muscle microenvironment. Our present study demonstrates that canonical NF- $\mathrm{BB}$ signaling is important for satellite cell homeostasis and function during skeletal muscle regeneration. These finding are also supported by our recently published articles demonstrating that the targeted ablation of TRAF6 or TAK1, the upstream activator of IKK $\beta$, also inhibits satellite stem cell homeostasis and function [117, 147]. A more dramatic effect of satellite cell-specific ablation of TAK1 or TRAF6 compared to IKK $\beta$ on regenerative myogenesis could be attributed to the fact that, being upstream signaling modules, TAK1 and TRAF6 can regulate other pathways, including MAPKs, which are also implicated in the regulation of satellite cell fate and function [117, 147, 161]. 
In summary, our study provides initial evidence that canonical NF- $\mathrm{\kappa B}$ signaling promotes the proliferation and survival of satellite cells. Furthermore, we provide evidence that satellite cell expression of IKK $\beta$ is important for the successful regeneration of adult skeletal muscle. An exhaustion of satellite cells is common in several muscular disorders and conditions, both in actual number, as well as functional satellite cells [162]. However, it remains to be elucidated whether a reduction in NF- $\mathrm{kB}$ is responsible for satellite cell dysfunction in disease conditions. It will be interesting to investigate whether there is differential regulation of canonical NF- $\mathrm{BB}$ signaling in satellite cells and myofibers in different physiological and pathological conditions. Indeed, other myogenic pathologies, such as rhabdomyosarcoma, demonstrate aberrations in the canonical NF-kB signaling pathway [121]. Further studies into differential expressions of the canonical NF- $\mathrm{kB}$ signaling pathway may pave the road for novel therapies for pathological conditions involving skeletal muscle. 
FIGURE 2.1

A.

\begin{tabular}{|c|c|c|}
\hline Naïve & 5d-injured & \multirow{3}{*}{$\begin{array}{l}\leftarrow \text { IKK } \beta \\
\leftarrow \text { phospho-IKB } \alpha\end{array}$} \\
\hline$\rightarrow$ & $-\cdots \cdots$ & \\
\hline$\sqrt{1+1}$ & 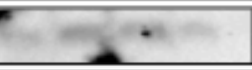 & \\
\hline$-1-+-4$ & $-\infty-\infty$ & $\leftarrow \mathrm{I} \kappa \mathrm{B} \alpha$ \\
\hline$--1-1$ & $--\cdots$ & $\leftarrow$ phospho-p65 \\
\hline---- & $-\infty-\infty$ & $\leftarrow$ p 65 \\
\hline & 6 & $\leftarrow$ GAPDH \\
\hline
\end{tabular}

B.

Anti-Pax 7
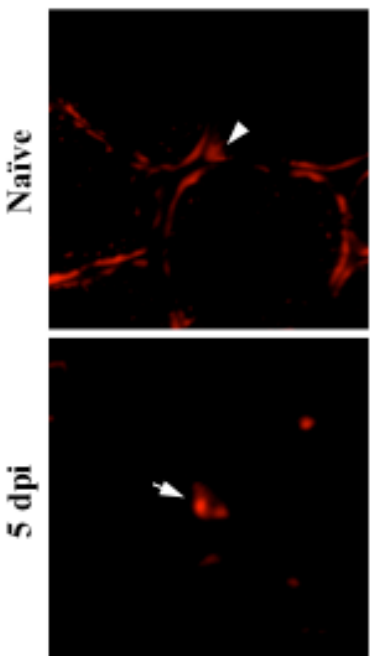

Arrow Head: Pax $7^{+}$p-p65
Anti-p-p65
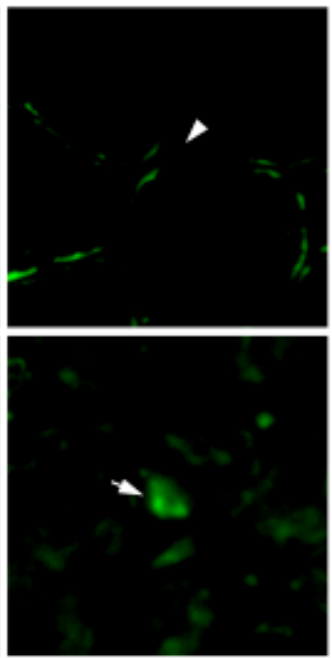

Arrow: Pax $7^{+}$p-p65
DAPI
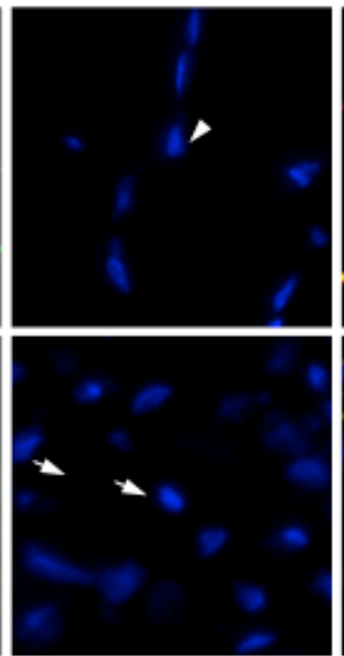

DAPI
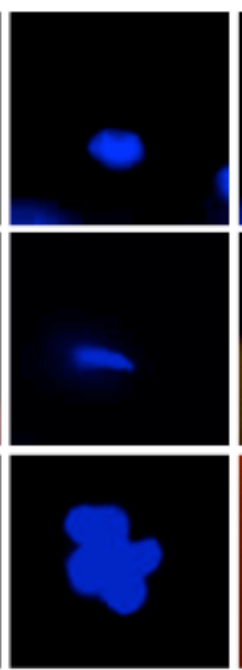

Merged
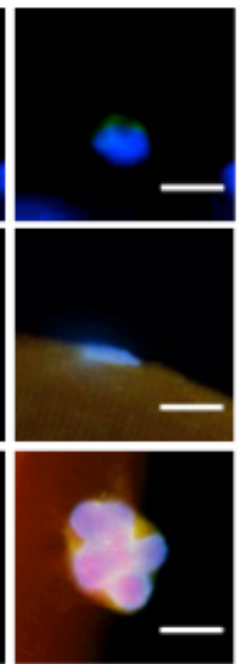

Scale Bar $=10 \mu \mathrm{m}$

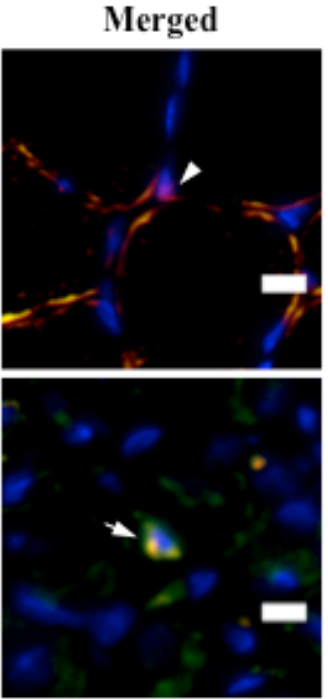

Scale Bar $=\mathbf{2 0} \mu \mathrm{m}$

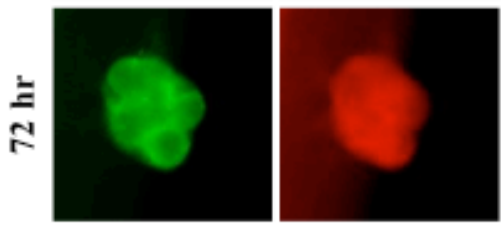




\section{FIGURE 2.1 Canonical NF-кB signaling is activated during regenerative}

myogenesis. TA muscle of 12-week old WT mice was injected with $100 \mu$ of $1.2 \%$

$\mathrm{BaCl}_{2}$ while the other side was injected with saline to serve as the uninjured control (A) Representative Immunoblots demonstrating the levels of: IKK $\beta$, phospho- and total I $\mathrm{B} \alpha$ and p65, and unrelated protein GAPDH in uninjured and 5-day injured TA muscle. (B) In a separate experiment, muscles were collected and processed for histological analysis. Representative photomicrographs of Pax7 and anti-phospho-p65 stained muscle sections from uninjured and injured TA muscle of WT mice. Nuclei were identified by staining

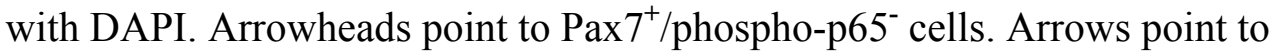
Pax $7^{+} /$phospho-p $65^{+}$cells. Scale bar, $20 \mu \mathrm{m}$. (D) Representative photomicrographs of EDL single myofiber suspension cultures at 0,24 , and $72 \mathrm{~h}$ incubation in growth medium after immunostaining for Pax7 and phospho-p65. Nuclei were identified by staining with DAPI. Scale bar, $10 \mu \mathrm{m}$. 


\section{FIGURE 2.2}

A.

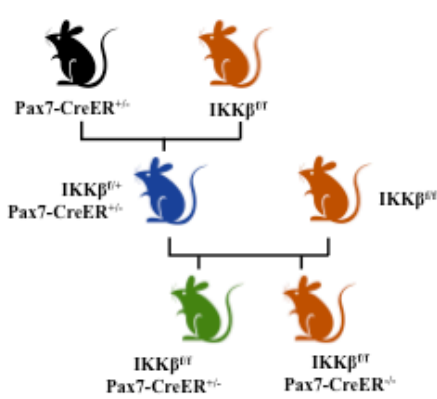

C.

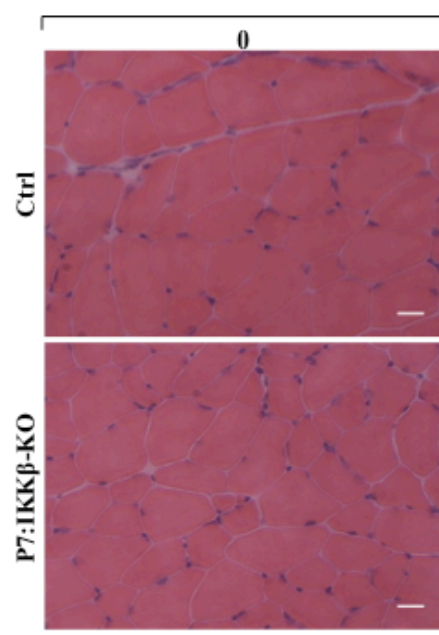

D.

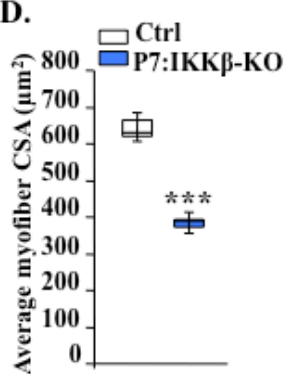

B.

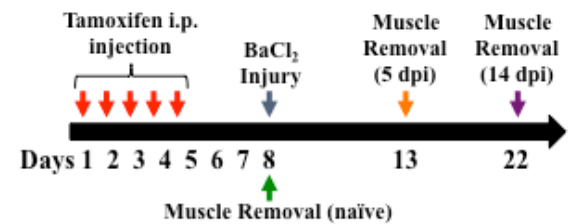

Time after injury (days)
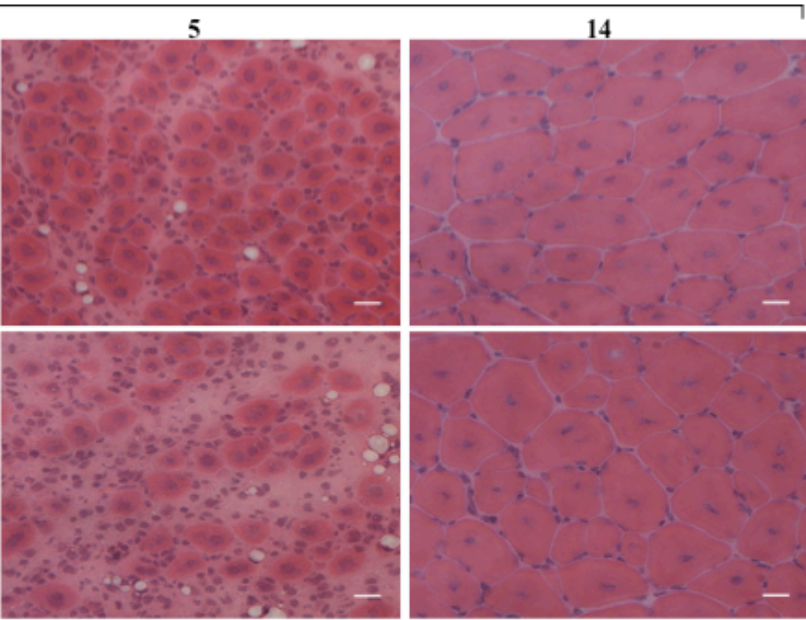

E.

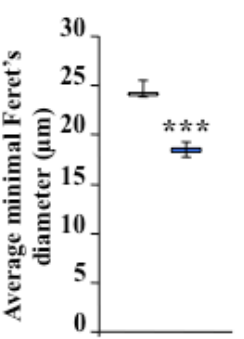

F.

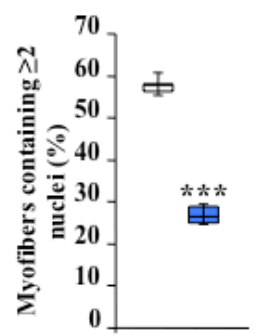

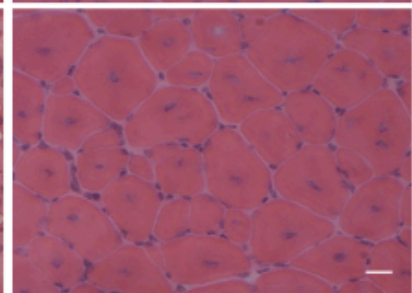

G.

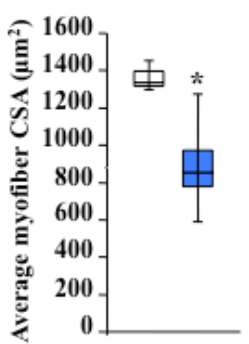

H.

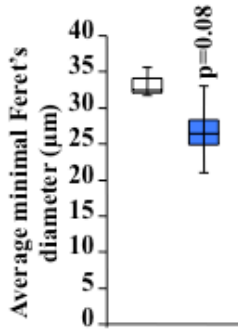




\section{FIGURE 2.2 Satellite cell-specific deletion of IKK $\beta$ impairs muscle regeneration in}

adult mice. (A) Schematic representation of the breeding strategy used for the generation of satellite cell-specific IKK $\beta$-knockout mice. (B) Treatment protocol for tamoxifeninduced Cre recombination and subsequent muscle collection in P7:IKK $\beta$-KO and Ctrl mice. (C) Representative photomicrographs of H\&E-stained sections illustrating a regeneration defect in injured TA muscle of $\mathrm{P} 7: \mathrm{IKK} \beta-\mathrm{KO}$ compared with littermate $\mathrm{Ctrl}$ mice at indicated time points after $\mathrm{BaCl}_{2}$ injection. Scale bars: $20 \mu \mathrm{m}$. Quantification of (D) average myofiber CSA, (E) average minimal Feret's diameter, and (F) percentage of myofibers containing two or more centrally located nuclei per field at day 5 post-injury. $\mathrm{N}=5$. Quantification of $(\mathbf{G})$ average CSA and $(\mathbf{H})$ minimal Feret's diameter at day 14 post-injury. $\mathrm{N}=3(\mathrm{Ctrl}), 4(\mathrm{P} 7: \mathrm{IKK} \beta-\mathrm{KO}) .{ }^{*} \mathrm{P}<0.05, * * \mathrm{P}<0.01, * * * \mathrm{P}<0.001$ values significantly different from corresponding TA muscle of Ctrl mice by unpaired two-tailed t-test. 


\section{FIGURE 2.3}

A.

Ctrl

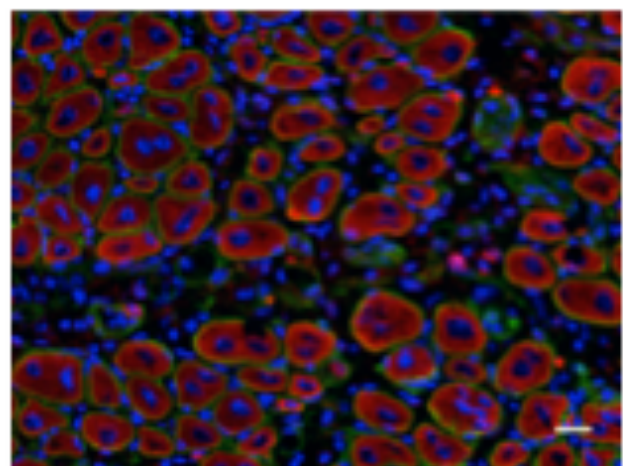

P7:IKKค-KO

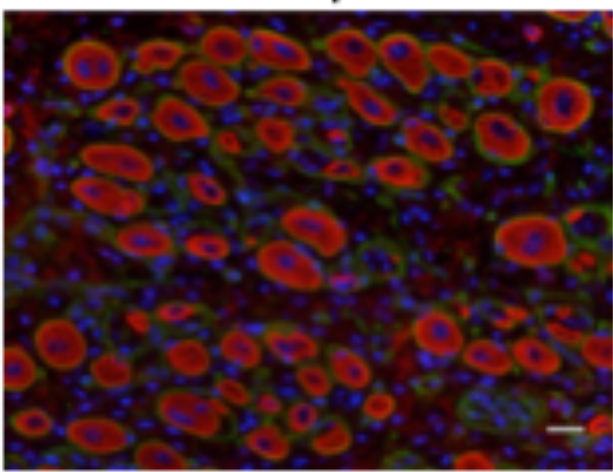

B.

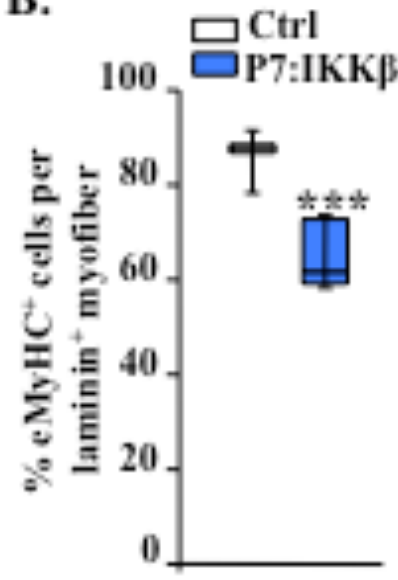

C.

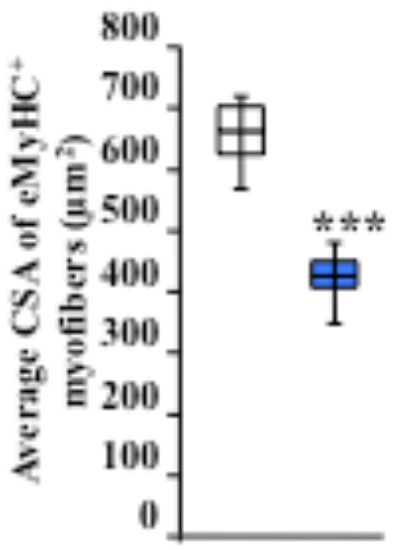

D.

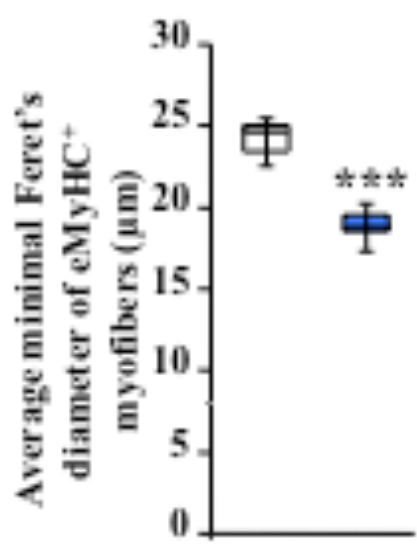




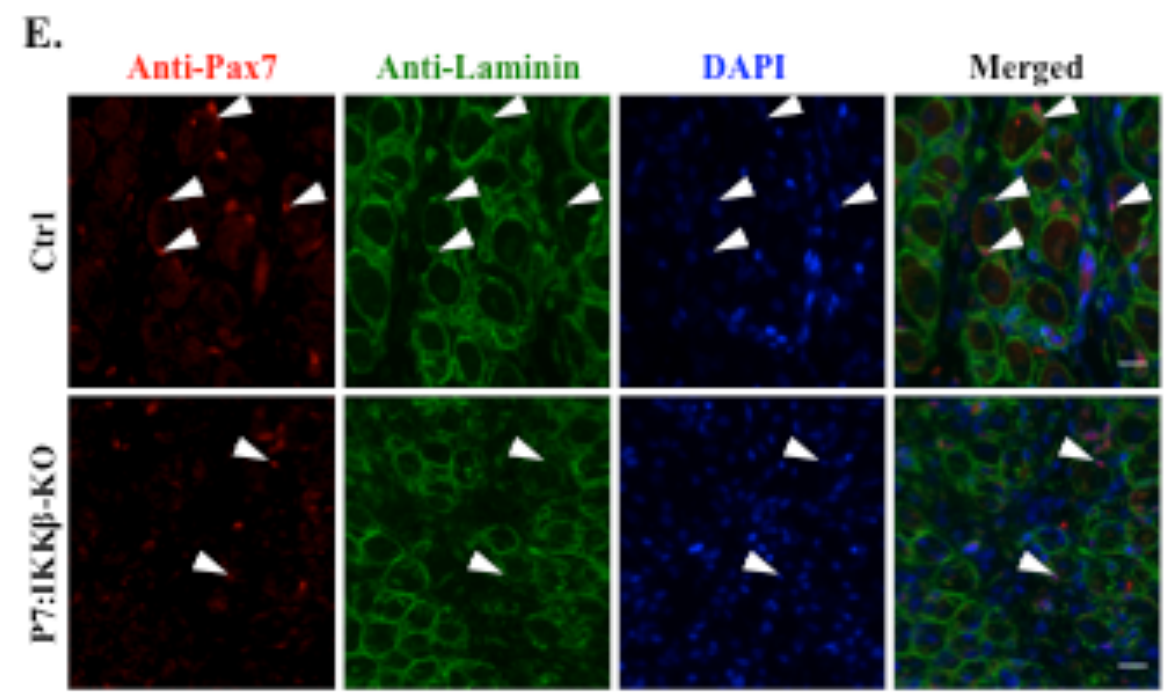

F.

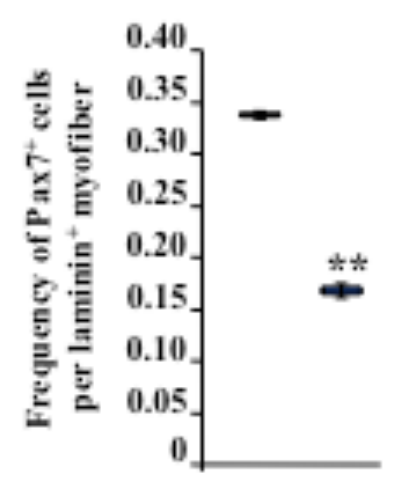

G.

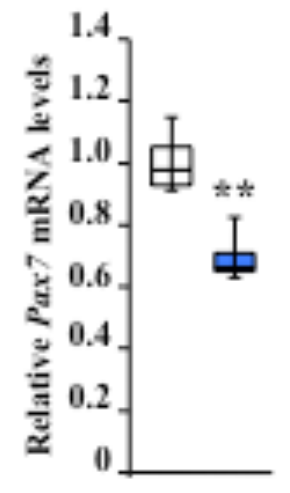

H.

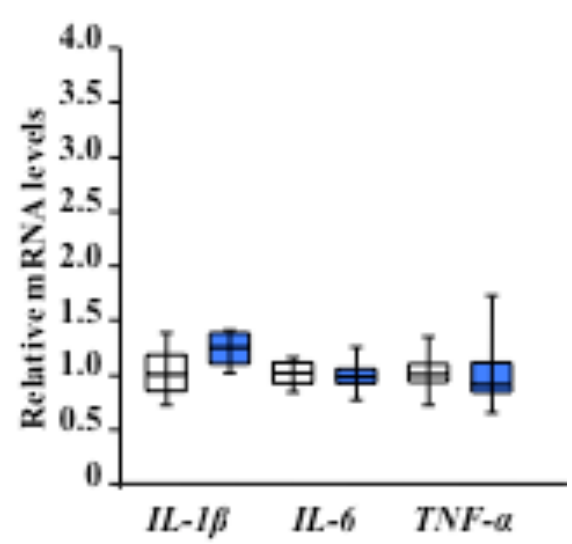


FIGURE 2.3 Inactivation of IKK $\beta$ in satellite cells impedes the formation of new myofibers and diminishes the satellite cell pool. (A) Representative photomicrograph of five day injured transverse TA muscle section of Ctrl and P7:IKK $\beta$-KO 12-week old mice after immunostaining for eMyHC and Laminin. Nuclei were identified by staining with DAPI. Scale bar, $20 \mu \mathrm{m}$. Quantification of $(\mathbf{B})$ percentage of eMyHC $\mathrm{My}^{+}$myofibers per Laminin $^{+}$myofiber, $(\mathbf{C})$ average CSA of eMyHC ${ }^{+}$myofiber, and (D) average minimal Feret's diameter of eMyHC ${ }^{+}$myofiber in five day injured $\mathrm{P} 7: \mathrm{IKK} \beta-\mathrm{KO}$ and $\mathrm{Ctrl}$ mice. (E) Representative photomicrograph of five day injured transverse TA muscle section of Ctrl and P7:IKK $\beta$-KO mice after immunostaining for Pax7 and Laminin. Nuclei were identified by staining with DAPI. Arrowhead points to Pax $7^{+}$cells. Scale bar, $20 \mu \mathrm{m}$. (F) Quantification of number of $\operatorname{Pax}^{+}$cells per Laminin ${ }^{+}$myofiber. $\mathrm{N}=5$. (G) Relative mRNA levels of Pax7 in 5 day injured TA muscle of P7:IKK $\beta-\mathrm{KO}$ and Ctrl mice. (H) Relative mRNA levels of select inflammatory markers in 5 day injured TA muscle of $\mathrm{P} 7: \mathrm{IKK} \beta-\mathrm{KO}$ and $\mathrm{Ctrl}$ mice. $\mathrm{N}=4 .{ }^{*} \mathrm{P}<0.05, * * \mathrm{P}<0.01, * * * \mathrm{P}<0.001$ values significantly different from corresponding TA muscle of Ctrl mice by unpaired two-tailed t-test. 


\section{FIGURE 2.4}

A.

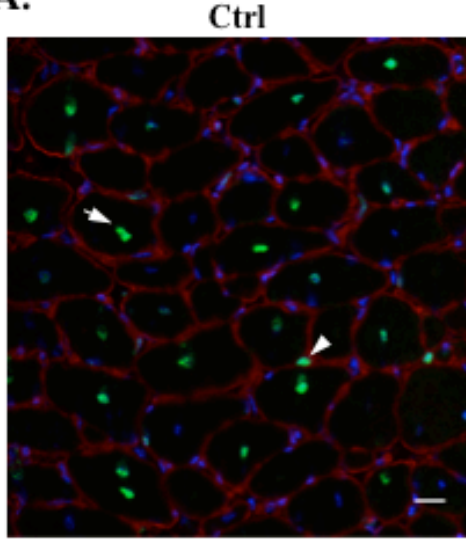

C.
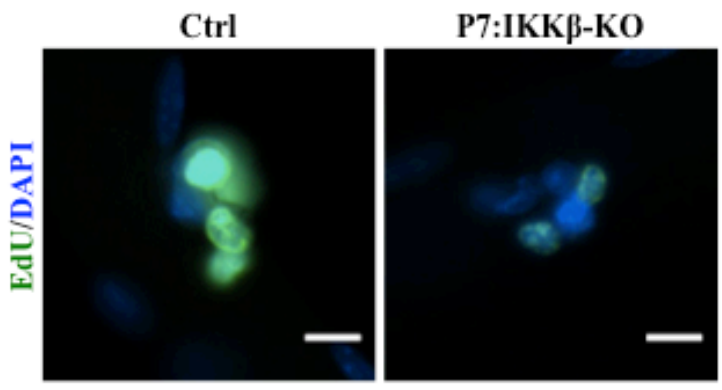

E.
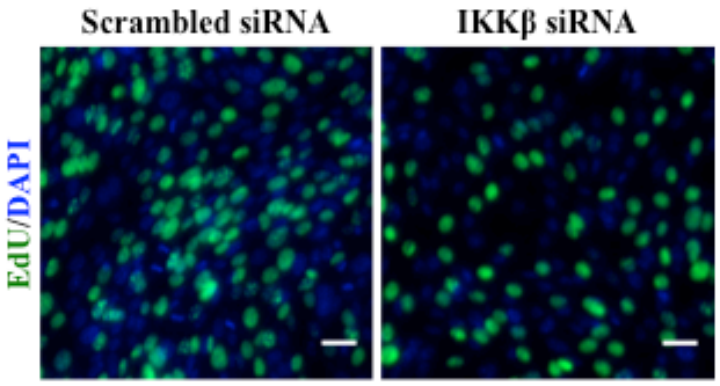

D.

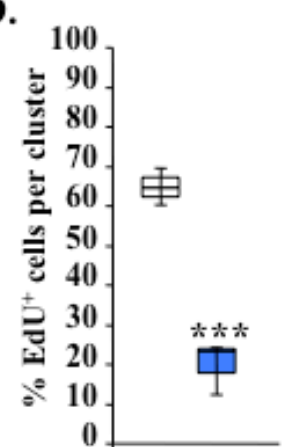

B.

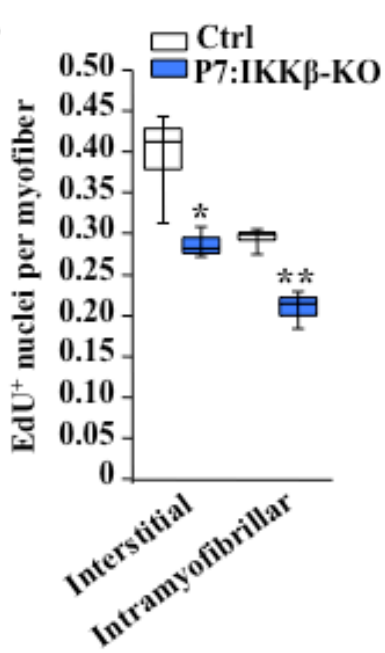

F.

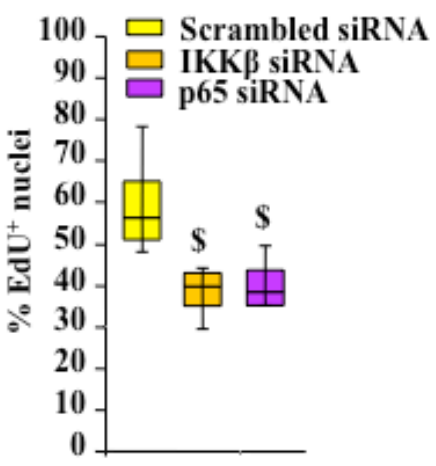

G.

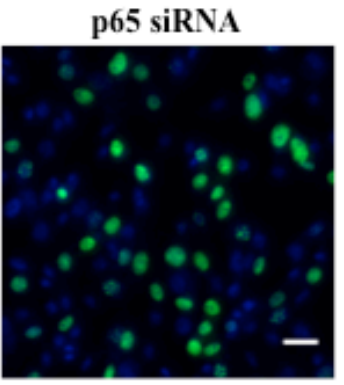




\section{FIGURE 2.4 Inactivation of IKK $\beta$ in satellite cells diminishes satellite cell}

proliferation. TA muscle of $\mathrm{P} 7: \mathrm{IKK} \beta-\mathrm{KO}$ and $\mathrm{Ctrl}$ mice was injured by intramuscular injection of $100 \mu \mathrm{l}$ of $1.2 \% \mathrm{BaCl}_{2}$ solution. After 2 days, the mice were given an intraperitoneal injection of EdU and 12 days later TA muscles were collected and muscle sections prepared were stained to detect EdU, laminin, and nuclei. (A) Representative photomicrograph of 14 day injured transverse TA muscle section of $\mathrm{Ctrl}$ and P7:IKK $\beta$ KO mice after processing for EdU and Laminin detection. Nuclei were identified by staining with DAPI. Arrowhead points to interstitial EdU ${ }^{+}$nuclei. Arrow points to intramyofibrillar EdU ${ }^{+}$nuclei. Scale bar, $20 \mu \mathrm{m}$. (B) Quantification of interstitial EdU $\mathrm{Ed}^{+}$ and intramyofibrillar EdU ${ }^{+}$nuclei per Laminin ${ }^{+}$myofiber in TA muscle of Ctrl and P7:IKK $\beta$-KO mice. N=4 (Ctrl), 3 (P7:IKK $\beta-K O)$. Single myofibers cultures were established from EDL muscle of Ctrl and P7:IKK $\beta$-KO mice and cultured for $72 \mathrm{~h}$. For last 90 min, the cells were pulse labeled with EdU by addition of EdU in culture medium. The myofibers cultures were then fixed with paraformaldehyde and stained for EdU. Nuclei were labeled by incubation in DAPI. (C) Representative merged images of EdU and DAPI staining are presented here. Scale bar, $10 \mu \mathrm{m}$. (D) Quantification of percentage of $\mathrm{EdU}^{+}$cells per cluster. Cluster was defined as $\geq 4$ cells. $\mathrm{N}=3$ mice, 9-11 fibers per group. Primary WT myoblasts were transfected with scrambled siRNA or siRNA targeting IKK $\beta$ or p65 for $72 \mathrm{~h}$. (E) Representative photomicrograph of siRNA transfected cells after processing for EdU detection. Nuclei were identified by staining with DAPI. Scale bar, $10 \mu \mathrm{m}$. (F) Quantification of percent EdU ${ }^{+}$cells. N=4. (G) In a similar experiment, cells were collected after $48 \mathrm{~h}$ of siRNA transfection and processed for biochemical analysis. Immunoblots represented here demonstrate the protein levels of 
Cyclin D1, Cyclin A, IKK $\beta$, p65, and unrelated protein GAPDH as a loading control. $\mathrm{N}=3 . * \mathrm{P}<0.05, * * \mathrm{P}<0.01,{ }^{* * *} \mathrm{P}<0.001$ values significantly different from corresponding TA muscle of Control mice by unpaired two-tailed t-test. ${ }^{\$} \mathrm{P}<0.05$, values significantly different from scrambled siRNA control by unpaired two-tailed t-test. 


\section{FIGURE 2.5}

A.

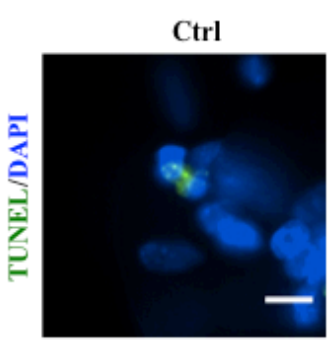

D.

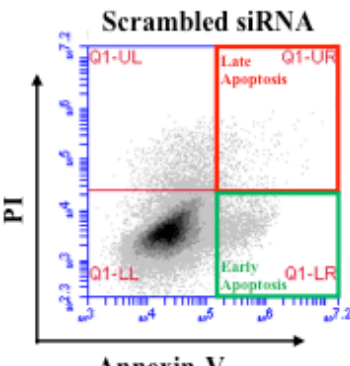

E.

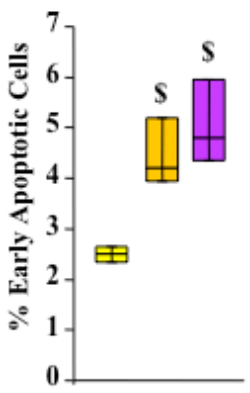

B.

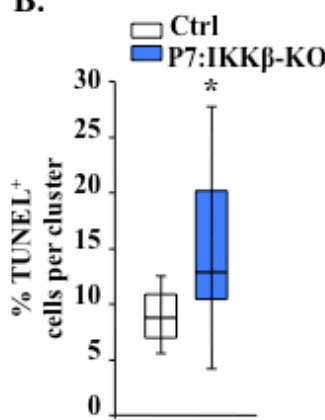

C. $\square$ Scrambled siRNA

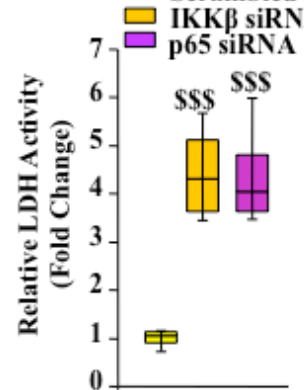

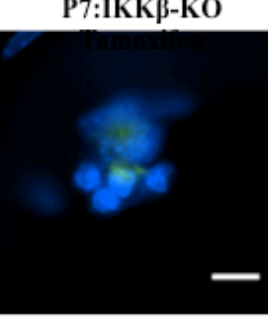

IKK $\beta$ siRNA
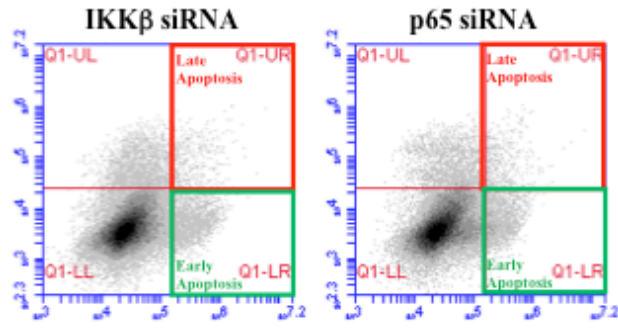

F.

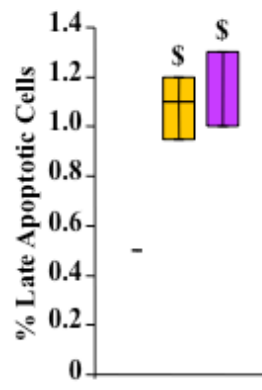

G.

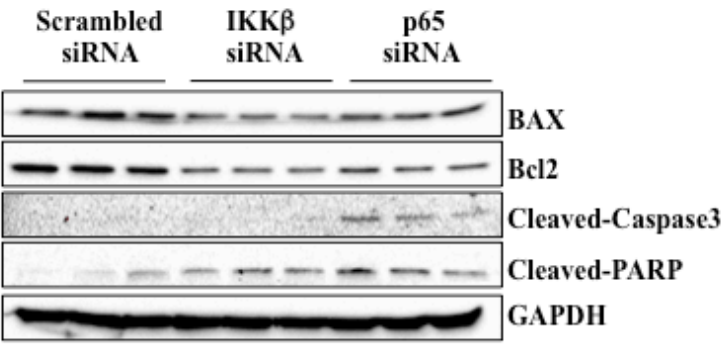




\section{FIGURE 2.5 Satellite cell-specific ablation of IKK $\beta$ causes an increase in apoptotic}

cell death. (A) Single myofiber suspension cultures were established from EDL muscle of 6-week old P7:IKK $\beta$-KO and Ctrl mice, and cultured for $72 \mathrm{~h}$ then processed for detection of TUNEL. Nuclei were identified by staining with DAPI. Scale bar, $10 \mu \mathrm{m}$. (B) Quantification of percentage of TUNEL ${ }^{+}$cells per cluster. Cluster was defined as $\geq 4$ cells. $\mathrm{N}=3$ mice, 9-11 fibers per group. Primary WT myoblasts were transfected with scrambled siRNA or siRNA targeting IKK $\beta$ or p65 for $48 \mathrm{~h}$ (C) Quantification of relative LDH activity (fold change) following siRNA transfection. (D) Following siRNA transfection, cells were collected and stained for Annexin V and propidium iodide (PI), and analyzed by FACS to detect early and late apoptotic cells. Quantification of (E) early (lower right quadrant) apoptotic cells and (F) late (upper right quadrant) apoptotic cells following siRNA transfection. (G) Immunoblots demonstrating the levels of cell death related proteins: BAX, Bcl2, Cleaved-Caspase3, Cleaved PARP and unrelated protein GAPDH. $\mathrm{N}=3 . * \mathrm{P}<0.05$, values significantly different from Scrambled siRNA transfected cells by unpaired two-tailed t-test. ${ }^{\$} \mathrm{P}<0.05$, values significantly different from scrambled siRNA control by unpaired two-tailed t-test. ${ }^{\$} \mathrm{P}<0.01$, values significantly different from scrambled siRNA control by unpaired two-tailed t-test. ${ }^{\$ \$} \mathrm{P}<0.001$, values significantly different from scrambled siRNA control by unpaired two-tailed t-test. 
FIGURE 2.6

A.
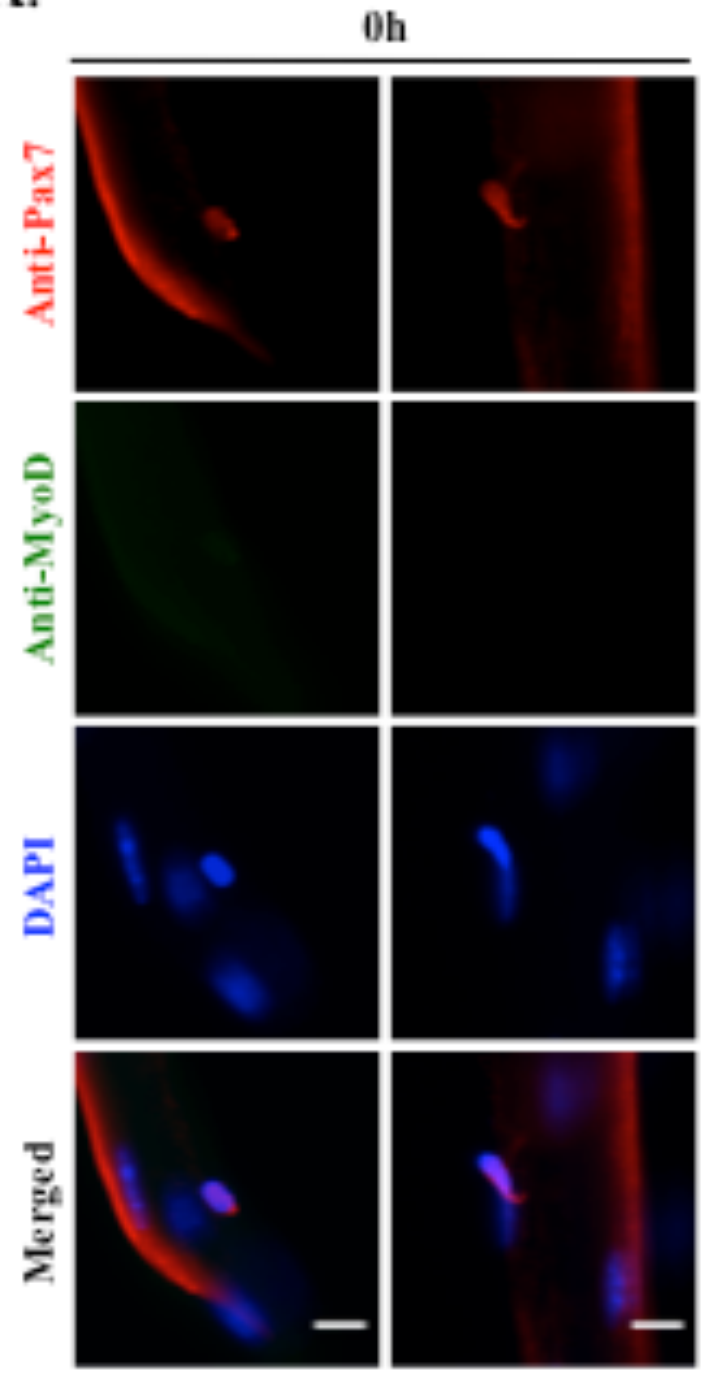

Ctrl
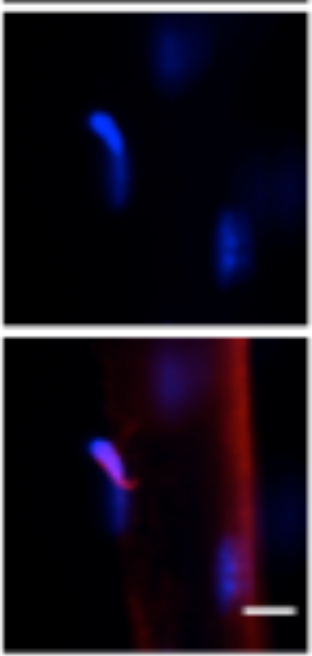

P7:IKK申-KO
B.

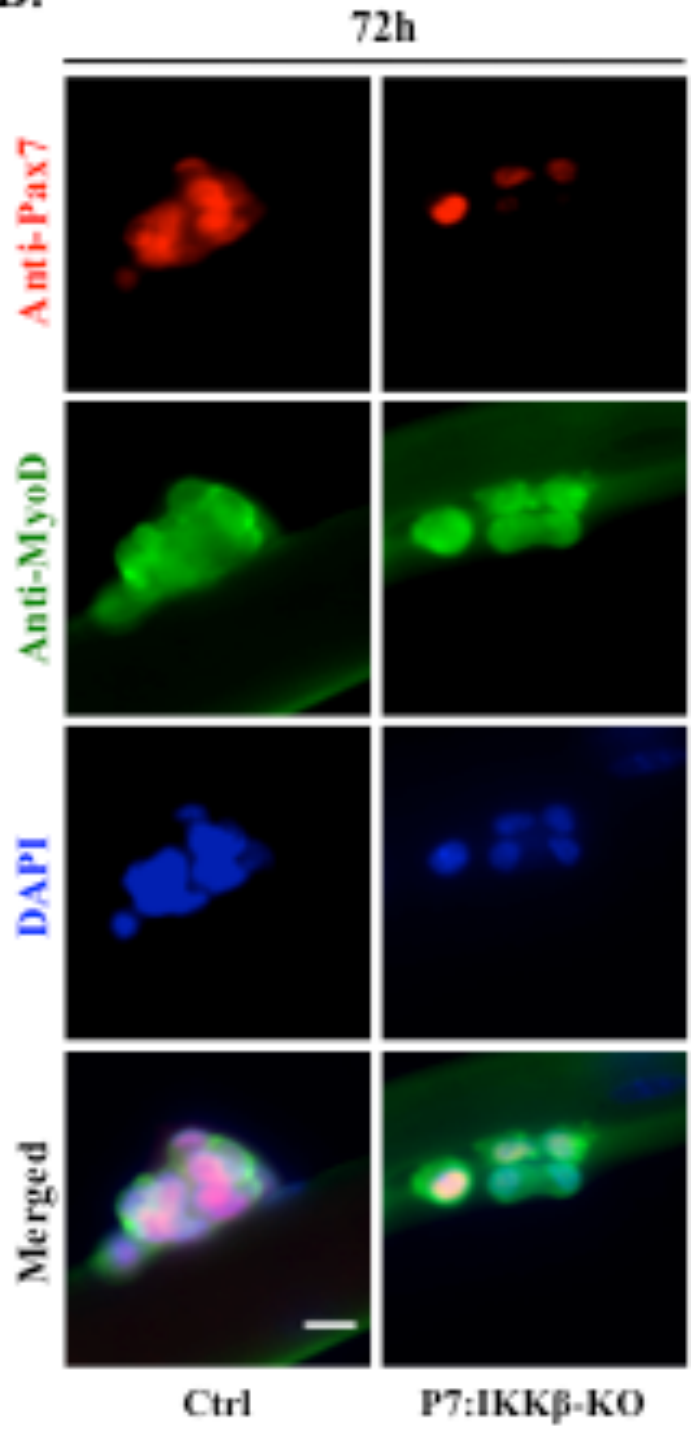


c.

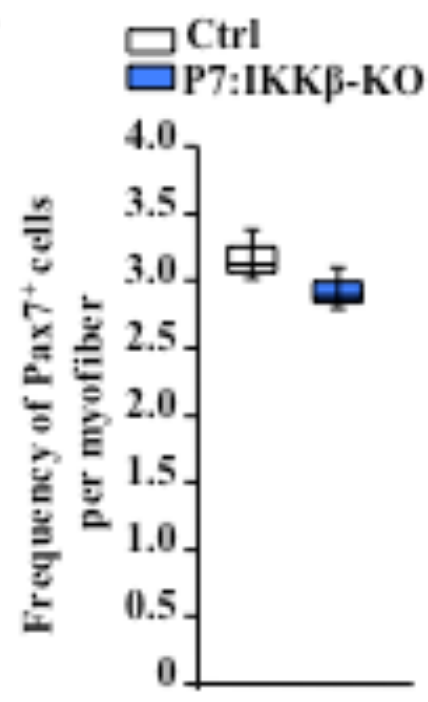

F.

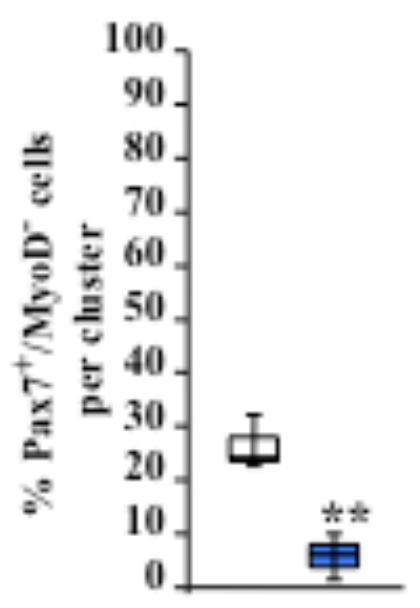

D.

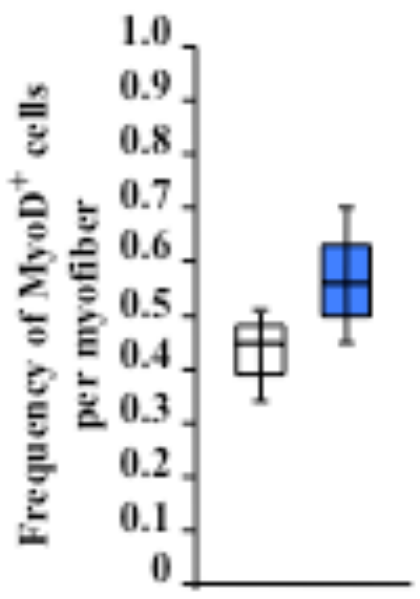

G.

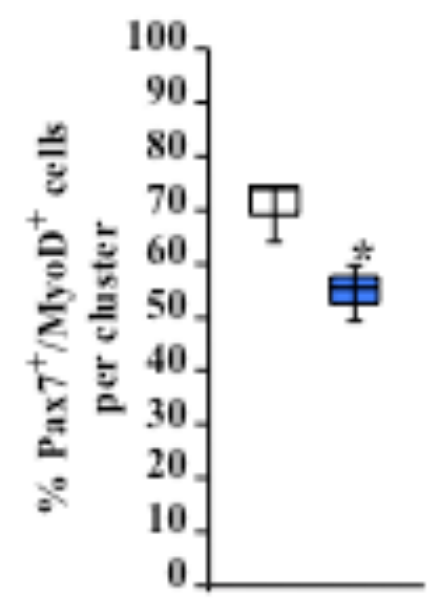

E.

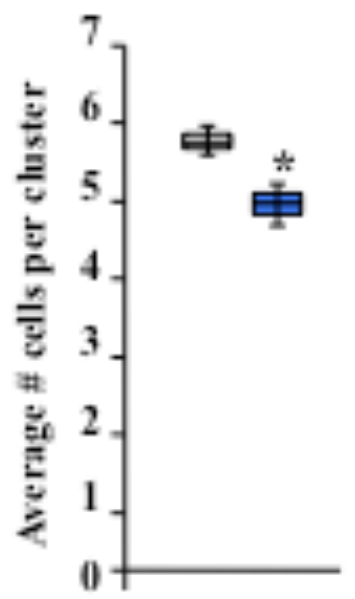

H.

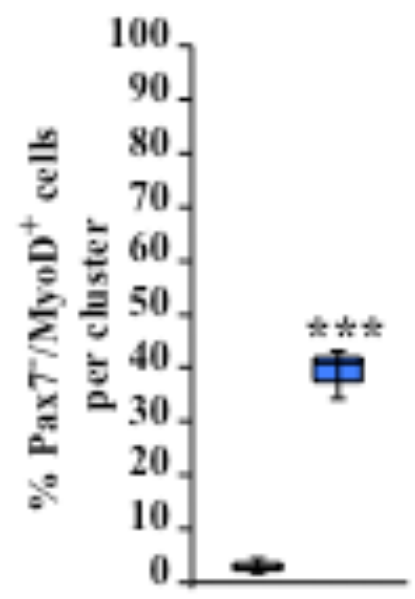




\section{FIGURE 2.6 Satellite cell-specific deletion of IKK $\beta$ diminishes the self-renewal of}

satellite cells and results in their premature differentiation. Single myofiber suspension cultures were established from EDL muscle of 6-week old P7:IKK $\beta-\mathrm{KO}$ and Ctrl mice, and cultured for 0 or 72 hours then collected and immunostained for Pax 7 and MyoD. Representative individual and merged images of (A) freshly isolated and (B) $72 \mathrm{~h}$ cultured myofibers from $\mathrm{Ctrl}$ and P7:IKK $\beta$-KO mice stained with PAX7, MyoD, and DAPI. Quantification of $(\mathbf{C}) \mathrm{Pax}^{+}$cells per myofiber and (D) $\mathrm{MyoD}^{+}$cells per myofiber in $\mathrm{Ctrl}$ and P7:IKK $\beta$-KO 0-hour cultures. Quantification of $(\mathbf{E})$ average number of cells per cluster, (F) percentage of $\mathrm{Pax}^{+} / \mathrm{MyoD}^{-}$cells per cluster, $(\mathbf{G})$ percentage of $\mathrm{Pax}^{+} / \mathrm{MyoD}^{+}$cells per cluster, and $(\mathbf{H})$ percentage of $\mathrm{Pax} 7^{-} / \mathrm{MyoD}^{+}$cells per cluster in Ctrl and P7:IKK $\beta$-KO 72-hour cultures. Cluster was defined as $\geq 4$ cells. $\mathrm{N}=3$ mice, 9-11 fibers per group. Data presented as box-and-whisker plot. $* \mathrm{P}<0.05$, $* * \mathrm{P}<0.01$, $* * * \mathrm{P}<0.001$ values significantly different from $\mathrm{Ctrl}$ mice by unpaired two-tailed t-test. 


\section{FIGURE 2.7}

A.

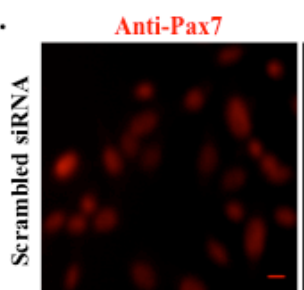

Anti-MyoD
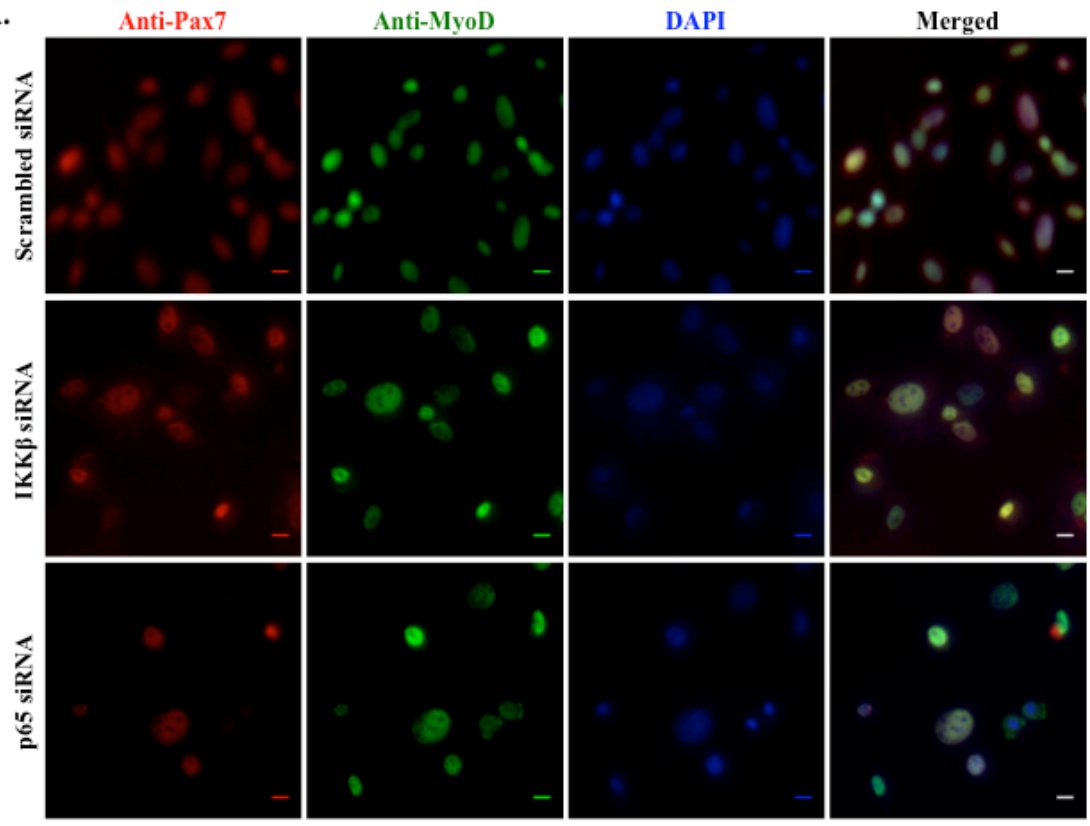

B.

C.

D.
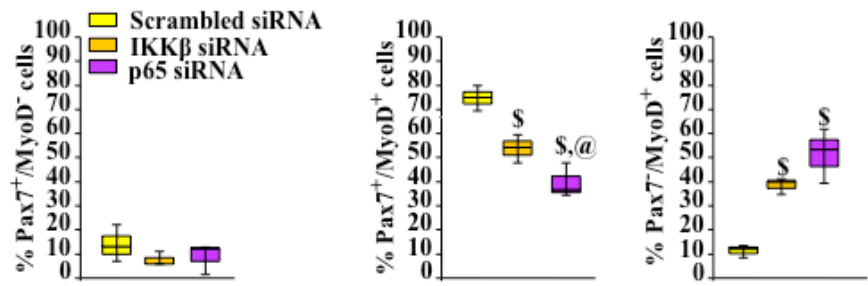

E.

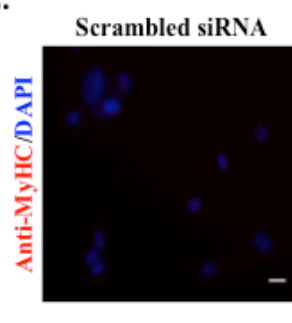

IKKB siRNA

p65 siRNA

F.

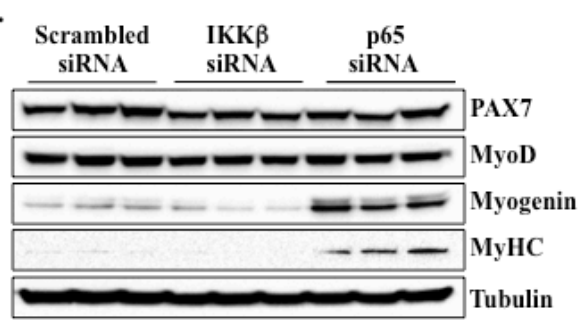




\section{FIGURE 2.7 Inhibition of IKK $\beta$ causes a precocious differentiation and inhibition}

of p65 results in an early terminal differentiation. Primary WT myoblasts were transfected with scrambled siRNA or siRNA targeting IKK $\beta$ or $\mathrm{p} 65$ for $72 \mathrm{~h}(\mathbf{A})$ Representative photomicrograph of siRNA transfected cells after immunostaining for Pax7 and MyoD. Nuclei were identified by staining with DAPI. Quantification of (B) percentage of $\mathrm{Pax}^{+} / \mathrm{MyoD}^{-}$cells, $(\mathbf{C})$ percentage of $\mathrm{Pax}^{+} / \mathrm{MyoD}^{+}$cells, and (D) percentage of $\mathrm{Pax}^{-} / \mathrm{MyoD}^{+}$cells post-siRNA transfection. $\mathrm{N}=3$. (E) Representative photomicrograph of siRNA transfected cells after immunostaining for MyHC. Nuclei were identified by staining with DAPI. In a similar experiment, WT myoblasts were transfected with scrambled siRNA or siRNA targeting IKKb or p65 for $48 \mathrm{~h}$. (F) Representative Immunoblots showing protein levels of Pax7, MyoD, Myogenin, MyHC, and unrelated protein Tubulin as a loading control. ${ }^{\$} \mathrm{P}<0.05$, values significantly different from scrambled siRNA control by unpaired two-tailed t-test. ${ }^{\circledR} \mathrm{P}<0.05$, values significantly different from IKK $\beta$ siRNA transfected group by unpaired two-tailed t-test. 


\section{CHAPTER 3}

\section{SATELLITE CELL-SPECIFIC OVEREXPRESSION OF A CONSTITUTIVELY ACTIVE MUTANT OF IKK $\beta$ HINDERS MUSCLE REGENERATION}

3.1 Introduction. Skeletal muscle is composed of myofibers which themselves arise from fusion of hundreds of myoblasts during embryological development [122]. Skeletal muscle has the ability to repair itself, which is attributed to the presence of a satellite stem cell population [23]. Following myofiber injury, several cell types are recruited at the site of injury to assist in the restoration of skeletal muscle structure [23]. While a few other cell types have been suggested to commit to myogenic fate, satellite cells are the main cell type that ensures skeletal muscle regeneration. Upon activation, these cells proliferate, differentiate, and eventually fuse to injured myofibers to accomplish repair [24]. Moreover, a small proportion of activated satellite cells return to a quiescent state and replenish the satellite cell pool in freshly regenerated muscle [124]. Pax7 transcription factor is essential for maintaining the stemness of satellite cells and their function during regenerative myogenesis $[125,126]$.

Nuclear Factor- $\kappa \mathrm{B}(\mathrm{NF}-\kappa \mathrm{B})$ family proteins are expressed in both immune and non-immune cell types [100]. As mentioned in previous chapters, the canonical NF- $\kappa \mathrm{B}$ pathway involves upstream activation of $\operatorname{IKK} \beta$, which causes phosphorylation of $\mathrm{I} \kappa \mathrm{B} \alpha$ protein. Phosphorylated IкB $\alpha$ protein is subsequently degraded through the proteasome 


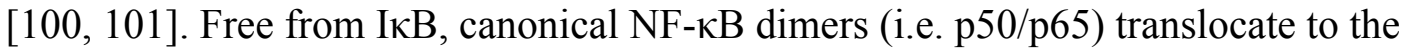
nucleus and orchestrate gene expression of several molecules involved in cellular survival, proliferation, and differentiation [101]. The canonical pathway promotes proliferation, but inhibits their differentiation into multinucleated myotubes $[108,110]$. By contrast, activation of the non-canonical NF- $\mathrm{KB}$ pathway requires upstream activation of NIK and IKK $\alpha$ [100]. IKK $\alpha$ facilitates the proteolytic processing of p100 into the p52 subunit, which then forms a complex with RelB. The p52/RelB complex eventually translocates to the nucleus to facilitate transcription of target genes [102]. Recent studies have suggested that the non-canonical NF- $\mathrm{kB}$ pathway promotes myoblast fusion. In addition, this pathway promotes mitochondrial biogenesis to improve the maintenance of the differentiated phenotype of myotubes [109].

Under naïve conditions, mature skeletal muscle exhibits a relatively low activity of the canonical NF-kB pathway. However, various components of this pathway have been reported to be upregulated in diseased skeletal muscle [119]. Indeed, inflammatory changes and an increase in oxidative stress in various skeletal muscle myopathies have been directly associated with heightened activation of canonical NF- $\mathrm{BB}$ signaling [119, 120]. Sarcopenia, or muscle wasting associated with age, has also been shown to be partially mediated by an increased activation of the canonical NF- $\kappa \mathrm{B}$ signaling cascade [111]. Furthermore, in a multitude of rhabdomyosarcoma substrains, primarily the alveolar subtype, the canonical NF-кB-YY1-miR-29 signaling axis is disrupted [121]. The miR-29 normally acts as a tumor suppressor, but due to the over-activation of the canonical NF-kB signaling pathway, miR-29 signaling is silenced leading to uncontrollable cell growth [121]. 
As such, modulating the activity of NF- $\mathrm{kB}$ signaling has been an experimental means of combating muscle pathology. In the $M d x$ mouse model of Duchenne muscular dystrophy, single allele deletion of NF- $\mathrm{BB}$ subunit p65/RelA was sufficient to reduce the infiltration of macrophages, myofiber necrosis, and calcification and improve the regeneration of injured myofibers in dystrophic muscle [160]. Similar results were also obtained upon shRNA-mediated knockdown of RelA or through the overexpression of a dominant negative mutant of IKK $\beta$ using adeno-associated virus (AAV) in skeletal muscle of $M d x$ mice [112]. Furthermore, targeted depletion of IKK $\beta$ in macrophages or mature myofibers also considerably reduced the expression of inflammatory cytokines and ameliorated the dystrophic phenotype in $M d x$ mice [113]. Consistently, muscle regeneration was also improved in WT mice lacking RelA or IKK $\beta$ in skeletal muscle following cardiotoxin mediated injury, as evidenced by increased numbers of centrally located nuclei and increased diameter of regenerating myofibers [102].

However, most of the investigations about the role of canonical NF-kB signaling in myopathy or myofiber regeneration have been carried out through modulating the levels of components of NF- $\mathrm{KB}$ in mature skeletal muscle or the inflammatory immune cells. A recent report has also suggested that an increase in NF- $\kappa B$ activity in satellite cells is also associated with age-associated defects in muscle repair [111]. While the role of canonical NF- $\mathrm{KB}$ signaling has become increasingly evidenced in the regulation of myogenesis, its role in satellite stem cell function remains less understood. Previous reports have shown that satellite cell activation of IKK $\beta$ results in a reduced differentiation through the myogenic lineage in select mouse models of cancer cachexia [116]. Work from our lab has shown that TWEAK, a pro-inflammatory cytokine, 
activates the canonical NF- $\kappa$ B pathway in satellite cells and that the inhibition of this pathway in TWEAK treated cultures resulted in an increase in the number of $\mathrm{Pax} 7^{+}$cells [140]. These studies and others indicate that depending on the cellular context or the stimulus, activation of the canonical NF- $\kappa \mathrm{B}$ signaling pathway results in a differential regulation of satellite cell fate.

Having derived from Chapter 2 that the satellite cell-specific depletion of IKK $\beta$ resulted in a decrease in muscle regeneration in mice, we next sought to investigate the effect of overexpression of IKK $\beta$ in satellite cells because of the signaling paradigm's close association with various skeletal muscle pathologies. Our results demonstrate that overexpression of a constitutively active mutant of IKK $\beta$ in satellite cells diminishes skeletal muscle regeneration following injury. IKK $\beta$ overexpressing satellite cells exhibit a precocious differentiation phenotype, but retain an intact self-renewal potential. We further report that forced activation of the canonical NF- $\kappa$ B pathway diminishes satellite cell proliferation and results in increased cell death. Thus, the results from the current study, coupled with our findings from the previous chapter, indicate that strict levels of

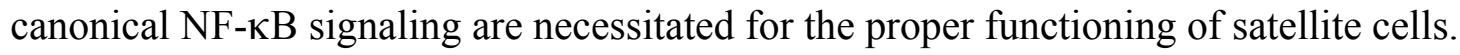

\subsection{Materials and methods.}

Animals. Satellite cell-specific inducible constitutive active (ca) IKK $\beta$ (i.e. P7:IKK $\beta$ ca) were generating by crossing Pax7-CreER with R26-Stop floxed IKK2ca mice (Jax Strain: B6(Cg)-Gt(ROSA)26Sor $\left.{ }^{\text {tm } 4(\mathrm{lkbkb}) R s k y} / \mathrm{J}\right)$. All mice were in the C57BL/6J background and their genotype was determined by PCR from tail DNA. For Cre-mediated inducible overexpression of IKK $\beta$, 6-week and 12-week old mice were injected i.p. with 
Tamoxifen (10 mg per Kg body weight) for five consecutive days. Control mice were injected with corn oil only. The IACUC and IBC of the University of Louisville approved all experimental protocols with mice in advance.

Skeletal muscle injury. One week after the first injection of tamoxifen, $100 \mu \mathrm{L}$ of $1.2 \%$ $\mathrm{BaCl}_{2}$ (Sigma Chemical Co.) in saline was injected into the TA muscle of mice to induce necrotic muscle injury. At various time points, TA muscle was collected from euthanized mice for biochemical and histological studies.

Myoblast fusion. To study myoblast fusion, two days following $\mathrm{BaCl}_{2}$-mediated necrotic muscle injury in the TA, mice were given an i.p. injection of 5-ethnyl-2'-deoxyuridine (EdU; $4 \mu \mathrm{g}$ per gm body weight). Twelve days post-EdU injection, the TA was isolated and transverse muscle sections made. The sections were subsequently immunostained with anti-Laminin, DAPI for the detection of nuclei, and processed for the detection of EdU. The number of interstitial and intramyofibrillar EdU ${ }^{+}$myonuclei per myofiber was quantified using NIH ImageJ software.

Histology and morphometric analysis. For skeletal muscle morphology and regeneration assessment, $10 \mu \mathrm{m}$-thick transverse sections of the TA were stained with Hematoxylin and Eosin. For quantitative analysis, CSA, minimum Feret's diameter, and multinucleation were analyzed in $\mathrm{H} \& \mathrm{E}$ stained TA muscle sections. H\&E slides were mounted using DPX Mountant For Histology Slide mounting medium (Sigma Chemical Co.) and visualized at room temperature on a Nikon Eclipse TE 2000-U Microscope 
(Nikon), a digital camera (Nikon Digital Sight DS-Fi1), and Nikon NIS Elements BR 3.00 software (Nikon). Exposure time was kept at $80 \mathrm{~ms}$ and contrast levels were not altered.

Satellite cell cultures. Satellite cells were isolated from the hind limbs of 6- to 8-weekold C57BL/6J mice as described [117]. For overexpression plasmid studies, cells were transfected using the Neon Electroporation transfection system using a protocol from the manufacturer with settings of: $1500 \mathrm{~V}, 10 \mathrm{~ms}$ pulse width, for three pulses. pCMV4 p65 was a gift from Warner Greene (Addgene plasmid \# 21966) [141]. pcDNA-IKK $\beta$-FLAG WT was a gift from Warner Greene (Addgene plasmid \# 23298) [142]. pcDNA3.1 (Invitrogen) was used as a control for the pCMV4 655 and pcDNA-IKK $\beta$-FLAG WT plasmids.

Isolation and culturing of myofibers. Single myofiber cultures were established from EDL muscle after digestion with collagenase II (Worthington Biochemical Corporation, Lakewood, NJ) and trituration as previously described [117]. Suspended myofibers were collected immediately or cultured in $60 \mathrm{~mm}$ horse serum-coated plates in Dulbecco's modified Eagle's medium (DMEM) supplemented with: 10\% fetal bovine serum (FBS; Invitrogen), $2 \%$ chicken embryo extract (Accurate Chemical, Westbury, NY), $10 \mathrm{ng} / \mathrm{mL}$ basic fibroblast growth factor (Peprotech, Rocky Hill, NJ), and 1\% penicillinstreptomycin for three days. 
Immunofluorescence. For IHC and ICC studies, frozen TA muscle sections ( 9 or $10 \mu \mathrm{m}-$ thick sections) or myoblast cultures, respectively, were fixed in $4 \%$ paraformaldehyde (PFA) in phosphate buffered saline (PBS), blocked in $2 \%$ bovine serum albumin (BSA) in PBS for one hour, and subsequently incubated with anti-Pax7 (1:5-1:10, DSHB Cat\# pax7, RRID:AB_528428), anti-eMyHC (1:200, DSHB Cat\# F1.652, RRID:AB_447163), anti-MyoD (1:200, Santa Cruz Biotechnology Cat\# sc-304, RRID:AB_631992), antiLaminin (1:150, Cell Signaling Technology Cat\# L9393, RRID:AB_477163), or anti-pp65 (1:100, Cell Signaling Technology Cat\# 3033, RRID:AB_331284) in blocking solution at $4{ }^{\circ} \mathrm{C}$ overnight under humidified conditions. The sections were washed briefly with PBS before incubation with respective secondary antibody for one hour at room temperature and then washed three times for five minutes with PBS. Nuclei were visualized by counterstaining with DAPI for five minutes. Refer to Appendix-3 for complete antibody listing.

EdU and TUNEL Staining. To determine the proliferation status of satellite cells, EdU staining was performed using a commercially available kit and following the protocol from the manufacturer (Click-iT EdU Cell Proliferation Assay kit, Invitrogen). Briefly, during the last 90 minutes of incubation, $10 \mu \mathrm{M}$ EdU (Invitrogen) was added in culture medium. The cells were fixed in 4\% PFA. EdU visualization occurs through a click reaction, whereby the EdU is covalently bonded to a fluorescent label (Alexa Fluor ${ }^{\circledR} 488$ Azide). Nuclei were visualized by counterstaining with DAPI for 5 minutes. TUNEL staining was performed following a protocol from the manufacturer (in situ Cell Death Detection Kit, Sigma Chemical Co.). Briefly, the sections or myofiber cultures were fixed 
in $4 \%$ PFA and permeabilized with $0.1 \%$ Triton $\mathrm{X}-100$ in $0.1 \%$ sodium citrate, and then incubated in TUNEL reaction mixture for 60 minutes at $37^{\circ} \mathrm{C}$.

Fluorescent Imaging. ICC/IHC slides were mounted using Aqua-Poly/Mount fluorescence medium (Polysciences, Inc) and visualized at $-0.4^{\circ} \mathrm{C}$ on a Nikon TiE 3000 Inverted Microscope (Nikon), a digital camera (DXM-1200C Coded Digital Camera), and Nikon NIS Elements AR software (Nikon). Image levels were equally adjusted using Adobe Photoshop CS6 software (Adobe). Detailed specifications, exposure times, and contrast levels provided in Appendix-4.

RNA isolation and quantitative Real-time PCR (qRT-PCR) Assay. RNA isolation and qRT-PCR were performed as previously described [117]. Total RNA was extracted from skeletal muscle tissue of mice or cultured myogenic cells using the TRIzol reagent (Thermo Fisher Scientific Life Sciences) and an RNeasy Mini Kit (Qiagen, Valencia, CA, USA) according to the manufacturers' protocols. First-strand cDNA for PCR analyses were generated with a commercially available kit (Thermo Fisher Scientific Life Sciences). Quantification of mRNA expression was performed using the SYBR Green dye (Thermo Fisher Scientific Life Sciences) method on a sequence-detection system (model 7300; Thermo Fisher Scientific Life Sciences). Approximately $25 \mu$ of reaction volume was used for the real-time PCR assays which consisted of $2 \times(12.5 \mu \mathrm{l})$ Brilliant SYBR Green QPCR Master Mix (Stratagene), $400 \mathrm{~nm}$ of primers ( $0.5 \mu$ l each from the stock), $11 \mu \mathrm{l}$ of water, and $0.5 \mu \mathrm{l}$ of template. The thermal conditions consisted of an initial denaturation at $95{ }^{\circ} \mathrm{C}$ for 10 min followed by 40 cycles of denaturation at $95{ }^{\circ} \mathrm{C}$ for 
$15 \mathrm{~s}$, annealing and extension at $60^{\circ} \mathrm{C}$ for $1 \mathrm{~min}$, and, for a final step, a melting curve of $95^{\circ} \mathrm{C}$ for $15 \mathrm{~s}, 60^{\circ} \mathrm{C}$ for $15 \mathrm{~s}$, and $95^{\circ} \mathrm{C}$ for $15 \mathrm{~s}$. All reactions were carried out in triplicate to reduce variation. Primers were designed using Vector NTI software (Thermo Fisher Scientific Life Sciences). The sequences of the primers used are as follows: $\boldsymbol{\beta}$ actin, 5'-CAG GCA TTG CTG ACA GGA TG-3' (forward) and 5'-TGC TGA TCC

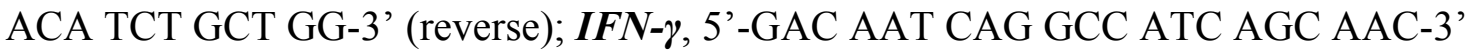
(Forward), 5'-CGG ATG AGC TCA TTG AAT GCT T-3'; IL-1/ , 5'-CTC CAT GAG CTT TGT ACA AGG-3' (Forward), 5'-TGC TGA TGT ACC AGT TGG GG-3' (Reverse); IL-6, 5'-ATG GCA ATT CTG ATT GTA TG -3' (Forward), 5'-TGG CTT TGT CTT TCT TGT TA-3' (Reverse); $\boldsymbol{P a x} 7$, 5' $^{\prime}$-CAG TGT GCC ATC TAC CCA TGC TTA-3' (Forward), 5'-GGT GCT TGG TTC AAA TTG AGC C-3' (Reverse); $\boldsymbol{M y f 5}$, 5'TGA AGG ATG GAC ATG ACG GAC G-3' (Forward), 5'-TTG TGT GCT CCG AAG GCT GCT A-3' (Reverse); $\boldsymbol{M y} \boldsymbol{h} 3$, 5'-ACA TCT CTA TGC CAC CTT CGC TAC-3' (Forward), 5'-GGG TCT TGG TTT CGT TGG GTA T-3' (Reverse); $\boldsymbol{M y} \boldsymbol{h} 4$, 5'-CGG CAA TGA GTA CGT CAC CAA A-3' (Forward), 5'-TCA AAG CCA GCG ATG TCC AA-3' (Reverse); MyoD, 5'-TGG GAT ATG GAG CTT CTA TCG C-3' (Forward), 5'GGT GAG TCG AAA CAC GGA TCA T-3' (Reverse); Myogenin, 5'-CAT CCA GTA CAT TGA GCG CCT A-3' (Forward), 5'-GAG CAA ATG ATC TCC TGG GTT G-3' (Reverse); and $\boldsymbol{T N F -} \boldsymbol{\alpha}, 5^{\prime}$-AGC ACA GAA AGC ATG ATC CG-3' (Forward), 5'-GCC ACA AGC AGG AAT GAG AA-3' (Reverse). The data were analyzed using SDS software version 2.0, and the results were exported to Microsoft Excel for further analysis. Data normalization was accomplished using two endogenous control ( $\beta$-actin) and the normalized values were subjected to a $2^{-\Delta \Delta \mathrm{Ct}}$ formula to calculate the fold change 
between the control and experimental groups. The formula and its derivations were obtained from the ABI Prism 7900 Sequence Detection System user guide.

Statistical analyses. For the sake of transparency, results were expressed as box-andwhisker plots with the box comprised of the $1^{\text {st }}, 2^{\text {nd }}$, and $3^{\text {rd }}$ quartiles, and the lower and upper whiskers corresponding to the minimum and maximum values, respectively, to display the entire range of data. Statistical analyses between two groups used unpaired two-tailed Student's t-test to compare quantitative data populations with normal distribution and equal variance. A value of $p<0.05$ was considered statistically significant unless specified otherwise for comparisons made between two groups.

\subsection{Results}

\subsubsection{Constitutive activation of canonical NF- $\mathrm{KB}$ signaling in satellite cells attenuates}

skeletal muscle regeneration. Our preceding results in Chapter 2 showed that the inhibition of canonical NF-KB signaling diminishes the regeneration of skeletal muscle in adult mice. However, there are also reports suggesting that the activation of the canonical NF- $\mathrm{BB}$ pathway, especially in disease conditions, reduces skeletal muscle regeneration $[116,129]$. To specifically investigate the effect of heightened canonical NF-kB signaling in satellite cell function during muscle regeneration, we employed R26Stop ${ }^{\mathrm{FL}}$ IKK2ca (henceforth Rosa26-IKK $\beta \mathrm{ca}$ ) mice that allow for the inducible expression of a constitutively active form of IKK $\beta$ (IKK $\beta \mathrm{ca}$ ) and subsequent activation of the canonical NF-KB pathway (Figure 3.1A) [163]. Rosa26-IKK $\beta$ ca mice were crossed with Pax7-CreER mice to generate Rosa26-IKK $\beta \mathrm{ca}$;Pax7-CreER mice. To induce the 
expression of IKK $\beta \mathrm{ca}$ in satellite cells, Rosa26-IKK $\beta \mathrm{ca} ; \mathrm{Pax} 7-\mathrm{CreER}$ mice were treated with tamoxifen or vehicle (corn oil) alone to generate satellite cell-specific IKK $\beta$ overexpression (henceforth P7:IKK $\beta \mathrm{ca}$ ) or control (Ctrl) mice, respectively (Figure 3.1B Overexpression of IKK $\beta$ in satellite cells of P7:IKK $\beta$ ca mice was confirmed through immunohistochemical staining of IKK $\beta$ protein (Figure 3.1C). Next, TA muscle of Ctrl and P7:IKK $\beta$ ca mice was injured unilaterally by intramuscular injection of $1.2 \% \mathrm{BaCl}_{2}$ solution and muscle regeneration was evaluated at day 5 or day 14 by performing $\mathrm{H} \& \mathrm{E}$ staining (Figure 3.2A). There was no significant difference in myofiber size in uninjured TA muscle of Ctrl and P7:IKK $\beta$ ca mice (Figure 3.2B, 3.2C). Interestingly, we found that the average myofiber CSA, as well as minimal Feret's diameter, were significantly reduced in TA muscle of P7:IKK $\beta$ ca mice compared with corresponding Ctrl mice (Figure 3.2D, 3.2E). Moreover, the proportion of myofibers containing two or more centrally located nuclei was also significantly reduced in 5d-injured TA muscle of P7:IKK $\beta$ ca mice compared with Ctrl mice (Figure 3.2F). However, after 14d of injury, muscle structure was restored in both genotypes, as the average myofiber CSA and minimal Feret's diameter were comparable between Ctrl and P7:IKK $\beta$ ca mice (Figure 3.2G, 3.2H). Collectively, these results suggest that constitutive activation of canonical

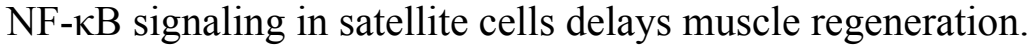

\subsubsection{Overexpression of IKK $\beta$ in satellite cells impedes the formation of new}

myofibers. To further examine the effect of IKK $\beta$ overexpression in satellite cell mediated muscle regeneration, we analyzed the proportion of myofibers expressing the embryonic isoform of myosin heavy chain (eMyHC). We did not find any myofibers 
expressing eMyHC in uninjured TA muscle of $\mathrm{Ctrl}$ or P7:IKK $\beta$ ca mice; consistent with the aforementioned notion that eMyHC is only expressed in newly generated myofibers (data not shown). Regenerating TA muscle from both groups was populated with newly formed eMyHC ${ }^{+}$myofibers 5 days following injury. (Figure 3.3A). However, the proportion of $\mathrm{eMyHC}^{+}$myofibers within the Laminin immunostaining was significantly reduced in the P7:IKK $\beta$ ca mice compared to littermate Ctrl mice (Figure 3.3B). Furthermore, the size of the $\mathrm{eMyHC}^{+}$myofibers, measured by the CSA and minimal Feret's diameter, was significantly reduced in regenerating muscle of P7:IKK $\beta \mathrm{ca}$ mice compared to Ctrl mice (Figure 3.3C, 3.3D).

We next investigated whether satellite cell-specific overexpression of IKK $\beta$ has any effect on the proportion of Pax7 expressing satellite cells. Uninjured and 5-day injured TA muscle of P7:IKK $\beta \mathrm{ca}$ and Ctrl mice were double immunostained for Pax7 and Laminin. Nuclei were counterstained with DAPI (Figure 3.3E). Under naïve-uninjured conditions, we found no significant changes in the number of satellite cells, evidenced by comparable levels of Pax7 between the two groups (data not shown). Interestingly, the number of satellite cells was significantly reduced in the TA muscle of 5d-injured P7:IKK $\beta$ ca compared to that of Ctrl littermate mice (Figure 3.3F). Consistently, we found a significant reduction in the mRNA levels of $\operatorname{Pax} 7$ in $5 \mathrm{~d}$-injured TA muscle of P7:IKK $\beta \mathrm{ca}$ compared to littermate Ctrl mice (Figure 3.3G). Altogether, these findings indicate that the overexpression of a constitutively active mutant of IKK $\beta$ dimnishes the pool of activated satellite cells following necrotic muscle injury.

Activation of the canonical NF-kB pathway has been shown to induce the expression of various proinflammatory cytokines $[\mathbf{1 2 7}, \mathbf{1 2 8}]$. However, it remains to be 
elucidated whether satellite cells are a source of inflammatory molecules during regenerative myogenesis. In an effort to investigate their possible involvement, we measured the transcript levels of the proinflammatory cytokines: Interleukin $(I L)-1 \beta, I L$ 6 , and Interferon- $\gamma(I F N-\gamma)$ via qRT-PCR. We found a significant reduction in the relative mRNA levels of all three proinflammatory cytokines in the 5 day injured TA muscle of P7:IKK $\beta$ ca compared to littermate Ctrl mice (Figure 3.3H), suggesting that the overexpression of IKK $\beta$ in satellite cells negatively regulates the expression of certain inflammatory cytokines in the context of regenerative myogenesis. IL-1 $\beta$ is a known activator of canonical NF-kB signaling and IL-6 has been shown to differentially regulate the canonical NF- $\mathrm{BB}$ signaling depending on cellular context [101, 164-166]. It is within the realm of possibility that heightened activation of NF-kB signaling in satellite cells results in feedback inhibition of its potential activators/mediators (i.e. IL-1 $\beta$ and IL-6) in an attempt to blunt the hyperactivated pathway.

\subsubsection{Overexpression of IKK $\beta$ in satellite cells leads to a reduction in satellite cell}

proliferation. To understand the cellular mechanisms for the reduction in satellite cells in skeletal muscle undergoing regeneration in P7:IKK $\beta \mathrm{ca}$ mice, we investigated the role of supra-physiological activation of canonical NF- $\mathrm{KB}$ signaling in the proliferation of satellite cells in vivo, ex vivo, and in vitro. TA muscle of 12-week old Ctrl and P7:IKK $\beta$ ca mice was injured unilaterally by intramuscular injection of $1.2 \% \mathrm{BaCl}_{2}$ solution. Forty-eight hours later, the mice were given an i.p. injection of EdU. Twelve days later, corresponding to 14 days post-injury, we investigated the number of EdU incorporating nuclei in the TA muscle (Figure 3.4A). Interestingly, we observed a 
significant reduction in both the interstitial and intramyofibrillar $\mathrm{EdU}^{+}$nuclei in TA muscle of P7:IKK $\beta$ ca mice compared with corresponding Ctrl mice (Figure 3.4B, 3.4C). To further understand whether supra-physiological IKK $\beta$-mediated NF- $\mathrm{KB}$ signaling specifically affects the proliferation of satellite cells, we generated myofiber explant cultures and measured changes in the proliferation of myofiber-associated satellite cells via EdU incorporation following 72h of culturing (Figure 3.4D). Similar to in vivo results, we found a significant reduction in the proportion of EdU ${ }^{+}$myofiber-associated satellite cells in explant cultures generated from P7:IKK $\beta$ ca mice compared to littermate Ctrl mice explant cultures (Figure 3.4E).

We further investigated the role of an overstimulation of canonical NF- $\mathrm{KB}$ signaling in satellite cell-derived myogenic cultures, where the cells are not associated with myofibers. Primary myogenic cultures established from WT mice were transfected with vector alone (pcDNA3.1) or plasmids expressing WT IKK $\beta$ or p65 cDNA [141, 142]. After $72 \mathrm{~h}$, we employed an EdU incorporation assay to study cellular proliferation (Figure 3.4F). Overexpression of IKK $\beta$ and p65 resulted in a small, but significant reduction in EdU incorporation in myogenic cultures (Figure 3.4G) Collectively, these results suggest that the reduced number of satellite cell in regenerating muscle of P7:IKK $\beta$ ca mice is due in part to a cell-autonomous defect in proliferation.

\subsubsection{Satellite cell-specific overexpression of constitutively active IKKß promotes cell}

death. One of the major roles of the canonical NF- $\mathrm{KB}$ signaling is to promote cell survival. However, sustained activation of NF-KB can also lead to cell death through apoptosis or necroptosis $[127,128]$. Our attempts to perform double immunostaining for 
TUNEL and Pax 7 were to no avail, possibly due to the levels of Pax 7 being reduced in satellite cells undergoing apoptosis. Therefore, we once again generated ex vivo myofiber explants from EDL muscle of P7:IKK $\beta \mathrm{ca}$ and Ctrl mice to investigate the effect of supraphysiological activation of the canonical arm of NF- $\mathrm{kB}$ signaling on the survival of myofiber associated satellite cells. Following $72 \mathrm{~h}$ in culture, the proportion of apoptotic cells was determined by performing TUNEL labeling. (Figure 3.5A). Interestingly, we observed an increase in the proportion of myofiber-associated $\mathrm{TUNEL}^{+}$cells in explant cultures derived from P7:IKK $\beta$ ca mice compared to Ctrl derived explant cultures (Figure 3.5B).

We next investigated the effect of hyperactivated NF- $\mathrm{BB}$ signaling on the survival of myofiber-free myogenic cultures. WT primary myoblasts were transfected with vector alone or plasmids expressing WT IKK $\beta$ or p65 cDNA for $72 \mathrm{~h}$. LDH is a stable enzyme that is released upon cell death and accumulates in the culture supernatant [147]. Surprisingly, we found no significant changes in the relative levels of LDH in culture supernatants following IKK $\beta$ or p65 overexpression (Figure 3.5C).

\subsubsection{Overexpression of IKK $\beta$ in satellite cells causes a precocious differentiation of} satellite cells without affecting their self-renewal. It has become increasingly clear that Pax7 determines the fate of satellite cells in conjunction with different factors, such as MyoD. Indeed, the expression patterns of Pax7 and MyoD signify the myogenic state of satellite cells as quiescent $\left(\mathrm{Pax}^{7^{+}} / \mathrm{MyoD}^{-}\right)$, activated $\left(\mathrm{Pax} 7^{+} / \mathrm{MyoD}^{+}\right)$, or differentiated $\left(\mathrm{Pax}^{-} / \mathrm{MyoD}^{+}\right)$. We next investigated whether the overexpression of IKK $\beta$ has any role in the regulation the myogenic status of satellite cells. To model the satellite cell 
dynamics associated with regenerative myogenesis, we generated ex vivo suspension cultures of EDL myofibers from P7:IKK $\beta \mathrm{ca}$ and Ctrl mice. The ex vivo suspension cultures were analyzed after $0 \mathrm{~h}$ and $72 \mathrm{~h}$ of culturing by staining with Pax7 and MyoD (Figure 3.6A, 3.6B). We found no significant difference in the number of myofiberassociated satellite cells in cultures prepared from P7:IKK $\beta \mathrm{ca}$ and $\mathrm{Ctrl}$ mice at $0 \mathrm{~h}$ (Figure 3.6C). In both groups, we found a negligible number of $\mathrm{MyoD}^{+}$expressing satellite cells at $0 \mathrm{~h}$, however, no significant differences were detected between $\mathrm{Ctrl}$ and P7:IKK $\beta$ ca-derived explants (Figure 3.6D).

Satellite cells associated with the myofiber explants formed clusters by $72 \mathrm{~h}$ of culturing. There was no significant difference in the average number of clusters per myofiber (data not shown), nor in the average number of cells per cluster in P7:IKK $\beta \mathrm{ca}$ cultures compared to Ctrl cultures (Figure 3.6E). Furthermore, our analysis showed that the proportion of self-renewing satellite cells (i.e. $\mathrm{Pax}^{+} / \mathrm{MyoD}^{-}$) was also not significantly different between control and P7:IKK $\beta$ ca explant cultures (Figure 3.6F). Interestingly, the proportion of $\mathrm{Pax}^{+} / \mathrm{MyoD}^{+}$cells was significantly decreased, while cells that are $\mathrm{Pax} 7 / \mathrm{MyoD}^{+}$were significantly increased in cultures derived from P7:IKK $\beta$ ca mice compared to those derived from Ctrl mice (Figure 3.6G, 3.6H). These results suggest that supra-physiological activation of IKK $\beta$ causes precocious differentiation of activated satellite cells

We further investigated the role of IKK $\beta$-mediated canonical NF- $\mathrm{\kappa B}$ signaling in the regulation of myogenic fate of satellite cells in a myofiber-free environment. We first established satellite cell-derived primary myogenic cultures from hind limb muscles of WT mice. Myogenic cultures taken from early passages were transfected with empty 
vector or plasmids containing IKK $\beta$ or p65 cDNA. Following $72 \mathrm{~h}$ under growth conditions, the cultures were fixed and then double immunostained for Pax7 and MyoD. Nuclei were counterstained with DAPI (Figure 3.7A). Consistent with published reports, we found that the majority of cells were $\mathrm{Pax}^{+} / \mathrm{MyoD}^{+}$, whereas a small proportion was $\mathrm{Pax}^{+} / \mathrm{MyoD}^{-}$(Figure 3.7B). There was a significant reduction in the proportion of proliferating cells $\left(\mathrm{Pax}^{+} / \mathrm{MyoD}^{+}\right)$, while a significant increase was observed in the proportion of differentiated $\left(\mathrm{Pax} 7 / \mathrm{MyoD}^{+}\right)$cells following the IKK $\beta$ or $\mathrm{p} 65$ overexpression (Figure 3.7C, 3.7D) consistent with the notion that hyperactivation of canonical NF-אB signaling in satellite cells compromises their myogenic potential.

3.4 Discussion. Satellite cells normally exist in a quiescent state [123], but are rapidly activated following injury to skeletal muscle [35]. Similar to other adult stem cells, satellite cells are capable of maintaining a reserve pool of undifferentiated cells through self-renewal $[17,33,35]$. A reduction in the satellite cell pool has been linked to an impairment in the regeneration of skeletal muscle, such as observed in certain genetic muscle disorders and age-related muscle loss [148-150]. NF- $\mathrm{KB}$ plays a major role in the regulation of cell fate, survival, and differentiation of various cell types [127, 151]. Under chronic disease states, activation of the canonical NF- $\mathrm{KB}$ in skeletal muscle has been associated with a prolonged inflammatory response contributing to exacerbated myopathy $[127,128]$.

In Chapter 2, we showed that physiological levels of canonical NF-kB signaling in satellite cells promote skeletal muscle regeneration following injury. Here we demonstrate that the hyperactivation of canonical NF- $\mathrm{\kappa B}$ signaling in satellite cells also 
impairs their function during regenerative myogenesis (Figure 3.2, 3.3). These results are consistent with a recently published report demonstrating that the overexpression of a constitutively active mutant of IKK $\beta$ in satellite cells diminishes muscle repair after cryoinjury [132]. Indeed, we have previously reported that the overexpression of a constitutively active mutant of IKK $\beta$ induces oxidative stress and reduces survival of cultured satellite cells [147]. Moreover, sustained activation of the canonical arm of NF$\kappa \mathrm{B}$ signaling can also reduce skeletal muscle regeneration by preventing the differentiation of satellite cells into the myogenic lineage, similar to that reported during cancer cachexia [116]. Moreover, in the settings of degenerative muscle disorders, the activation of the canonical arm of NF- $\mathrm{\kappa B}$ signaling within satellite cells may also reduce their myogenic potential. Indeed, partial inhibition of NF- $\mathrm{kB}$ has been found to improve the engraftment of muscle-derived stem cells in dystrophic muscle of $M d x$ mice [58, 157]. It is notable that while we observed a deficit in muscle regeneration at early time points (i.e. day 5), skeletal muscle of P7:IKK $\beta$ ca mice eventually regenerate, such that there is no major difference in muscle structure at day 14 post-injury, suggesting that the impairment in satellite cell function brought about by the hyperactivation of the canonical NF- $\kappa \mathrm{B}$ pathway is neutralized by the modulatory activity of other factors/pathways that govern satellite cell function during muscle regeneration.

Skeletal muscle regeneration is a multi-faceted process that is regulated by numerous signals released from both myogenic and non-myogenic cells that infiltrate the muscle microenvironment following injury $[122,123]$. In response to stimulating cues, satellite cells undergo transcriptional and translational reprogramming, which in turn signals for their exit from quiescence into a metabolically activate state, that is conducive 
to their survival [124, 153-155]. Canonical NF-KB signaling is known to induce proliferation and survival through augmenting the expression of a vast array of cell cycle and cell growth regulatory molecules $[127,129]$. Prior reports have shown that canonical NF- $\mathrm{kB}$ signaling promotes the proliferation of myogenic cells through an up-regulation of cyclin D1 [156]. Surprisingly, our results demonstrate that the constitutive activation of IKK $\beta$ in satellite cells results in blunted proliferation (Figure 3.4) and survival (Figure 3.5) of myofiber associated satellite cells.

Cellular proliferation was also reduced upon overexpression of WT IKK $\beta$ and p65 in cultured primary myoblasts, although to a modest extent (Figure 3.5). By contrast, we did not observe any statistical significant differences in cellular survival under similar settings as evidenced by LDH activity (Figure 3.5). These results, while seemingly conflicting with our ex vivo experiments where we observed a significant increase in TUNEL labeled satellite cells of P7:IKKßca myofiber explants, may be due to the differences in methodology used to detect cell death. There is also the fact that in our in vivo model of IKK $\beta$ overexpression, a constitutively active mutant of IKK $\beta$ was employed, which happens to be resistant to upstream regulation and therefore guarantees hyperactivation of the NF- $\mathrm{kB}$ pathway. On the other hand, WT forms of both IKK $\beta$ and p65 were utilized for the in vitro experiments, where their overexpression might not yield a sustained activation of the pathway, as is the case in the constitutively active mutant of IKK $\beta$. Indeed, we have previously reported that the overexpression of IKK $\beta \mathrm{ca}$ in vitro resulted in increased cell death and oxidative stress in primary myogenic cultures [65].

Proper muscle regeneration requires the generation of a sufficient number of activated satellite cells prior to their fusion to facilitate muscle repair. If the pool of 
activated satellite cells does not meet the regeneration demands due to a defect in survival, activation, proliferation, or due to premature differentiation, restoration of muscle homeostasis is compromised. Consistent with this notion, our results demonstrate that forced activation of canonical NF- $\mathrm{kB}$ signaling in satellite cells blunts muscle repair, in part due to defective cellular proliferation and precocious differentiation (Figure 3.6

and 3.7). On the contrary, previous reports have demonstrated that constitutive activation of canonical NF- $\mathrm{BB}$ signaling in mature skeletal muscle results in an inhibition of myogenic differentiation $[129,133]$. The seemingly discrepant data between these studies and ours indicates that aberrations in NF- $\mathrm{KB}$ signaling have differential roles in the stem cell population and mature tissue. Indeed, even within the same cell type, we have

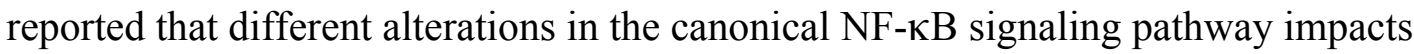
different facets of the myogenic process.

In summary, our study provides initial evidence that the conditional activation of the canonical NF- $\kappa \mathrm{B}$ signaling compromises the pool of activated satellite cells and impairs muscle regeneration. In conjunction with our knockout data, we conclude that strict monitoring of NF- $\kappa \mathrm{B}$ activity in satellite cells is important for the completion of regenerative myogenesis in adult skeletal muscle. Exhaustion in the satellite cell pool is observed in many skeletal muscle disorders and pathological conditions [162]. It remains

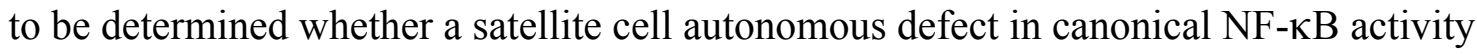
is associated with such myopathies. Further studies into the differential role of the canonical arm of NF- $\kappa \mathrm{B}$ signaling may pave the road for new therapies for skeletal muscle pathologies. 


\section{FIGURE 3.1}
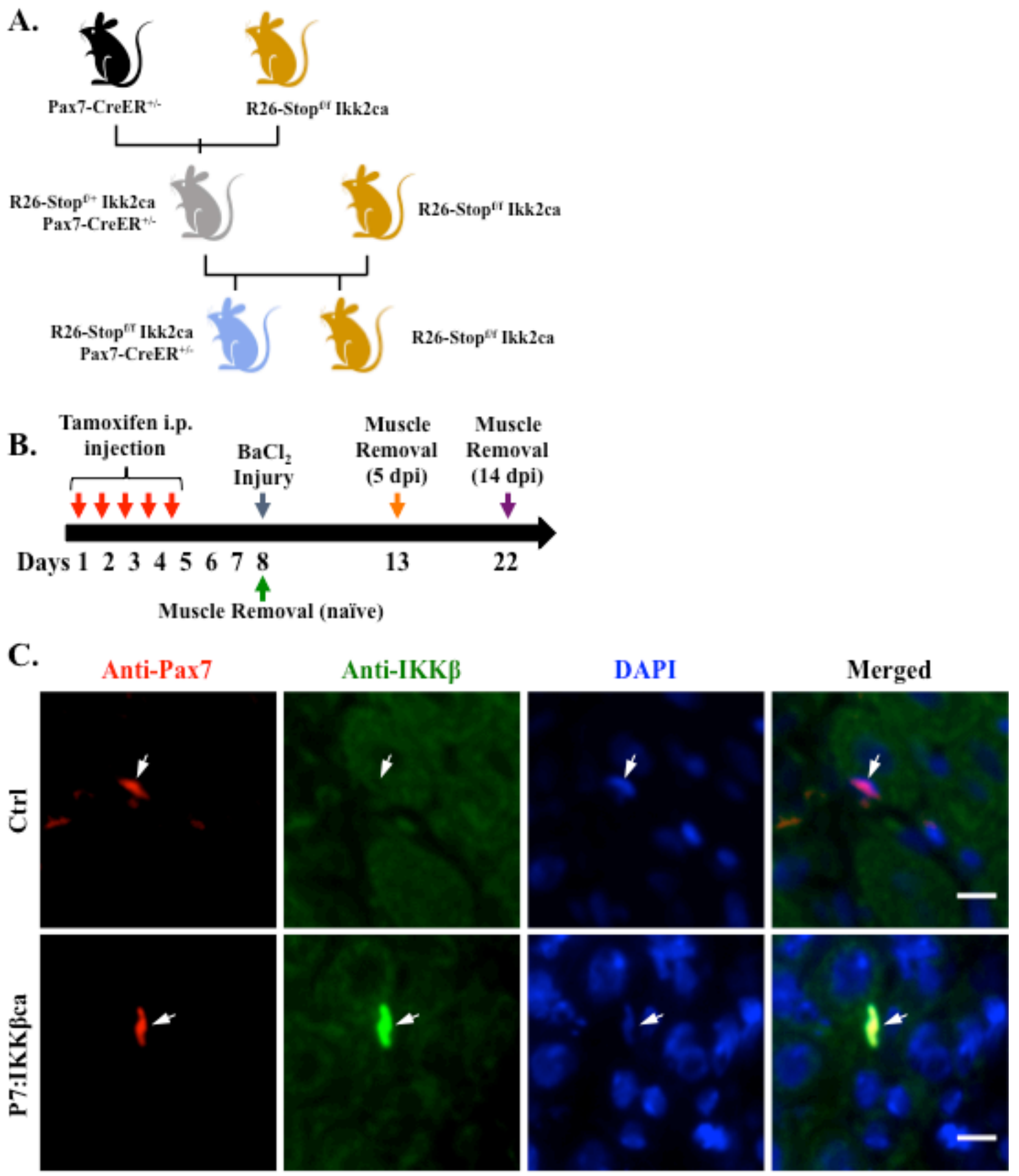


\section{FIGURE 3.1 Generation of satellite cell-specific inducible IKKßca overexpressing}

transgenic mice. (A) Schematic representation of the breeding strategy used for the generation of satellite cell-specific IKK $\beta$ ca mice (i.e. P7:IKK $\beta$ ca). (B) Schematic representation of treatment protocol for tamoxifen-induced Cre recombination and time points for $\mathrm{BaCl}_{2}$-mediated injury in TA muscle and subsequent muscle collection of P7:IKK $\beta$ ca and Ctrl mice. (C) Representative photomicrographs of 5d-injured transverse TA muscle sections of Ctrl and P7:IKK $\beta$ ca mice after immunostaining for Pax7 and IKK $\beta$. Nuclei were identified by staining with DAPI. Arrow points to nucleus of Pax $7^{+}$ cell. Scale bar, $10 \mu \mathrm{m}$. dpi, days post injury. 


\section{FIGURE 3.2}

A. Time after injury (days)
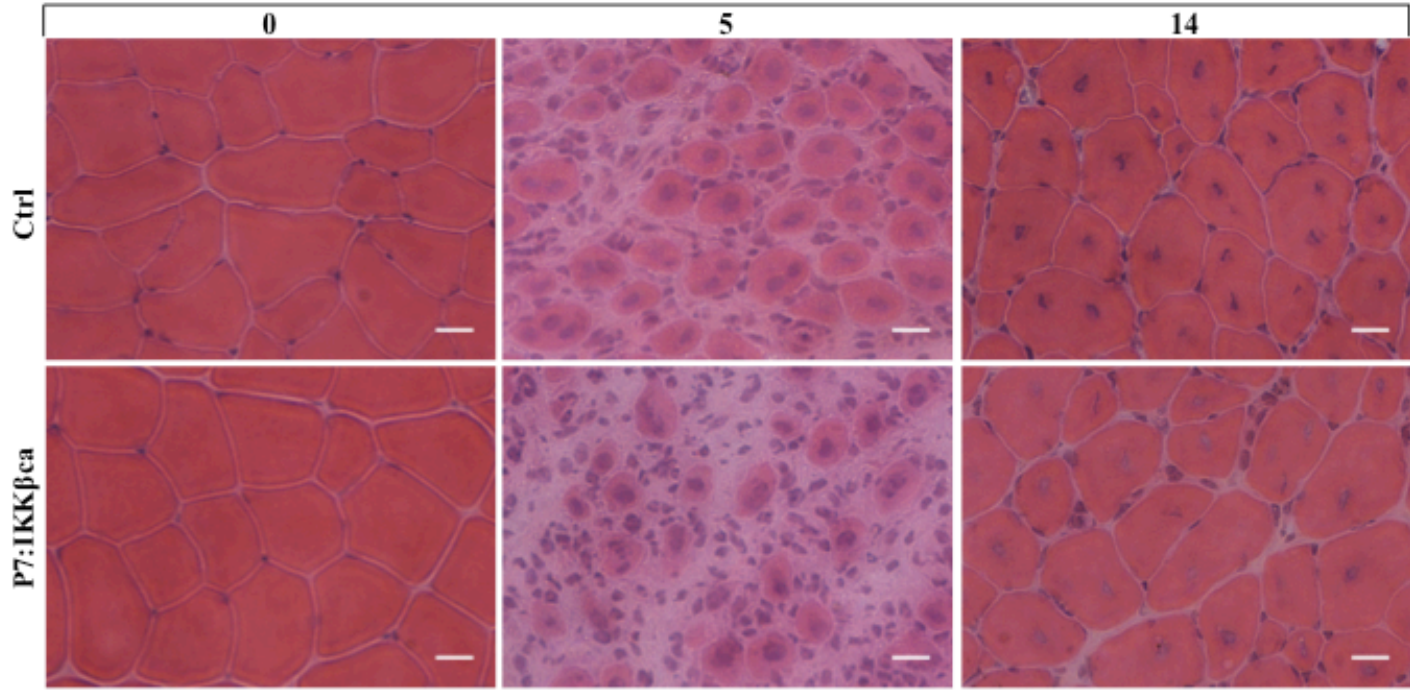

B.

C.
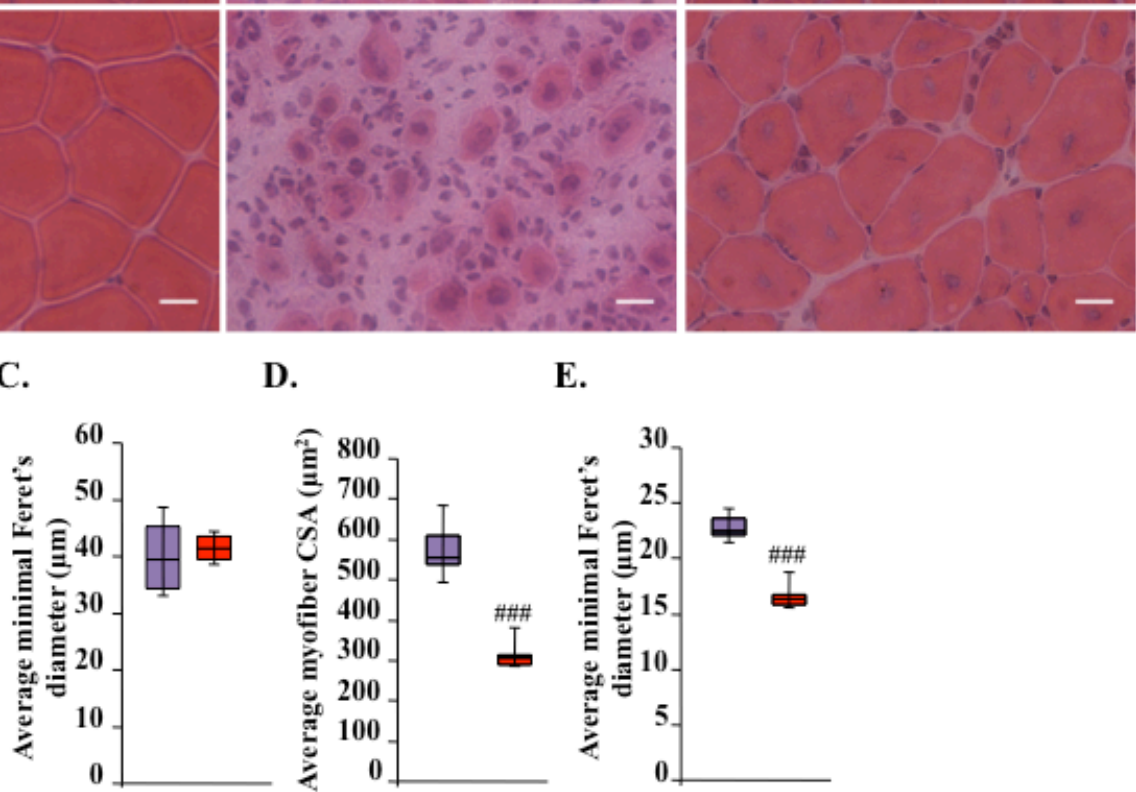

D.

E.
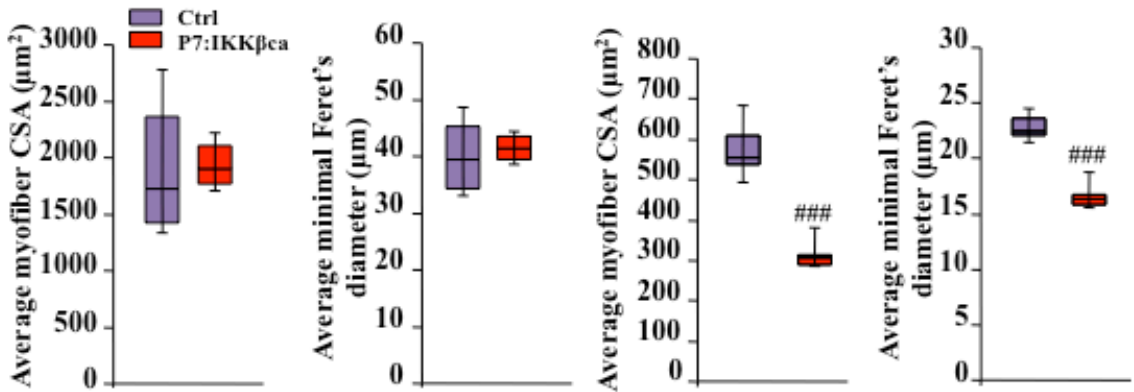

F.

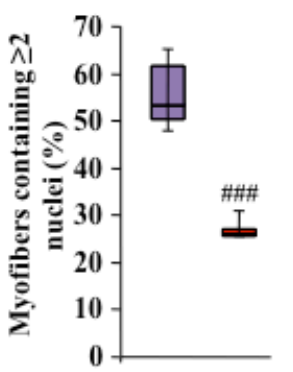

G.

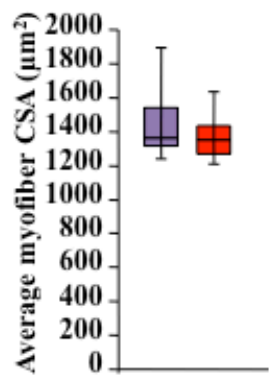

H.

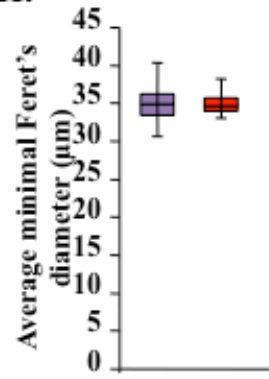




\section{FIGURE 3.2 Satellite cell-specific overexpression of IKKßca delays muscle}

regeneration in adult mice. (A) Representative photomicrographs of H\&E-stained sections illustrating regeneration defect in injured TA muscle of P7:IKK $\beta$ ca compared with littermate $\mathrm{Ctrl}$ mice at indicated time points after $\mathrm{BaCl}_{2}$ injection. Scale bars: $20 \mu \mathrm{m}$. Quantification of (B) average CSA and (C) minimal Feret's diameter under naïve conditions. $N=6$. Quantification of $(\mathbf{D})$ average myofiber CSA, $(\mathbf{E})$ average minimal Feret's diameter, and (F) percentage of myofibers containing two or more centrally located nuclei per field at day 5 post-injury. $\mathrm{N}=6$. Quantification of $(\mathbf{G})$ average myofiber CSA, and (H) minimal Feret's diameter at day 14 post-injury. $\mathrm{N}=6 .{ }^{\#} \mathrm{P}<0.05,{ }^{\# \#} \mathrm{P}<0.01$, ${ }^{\# \# \#} \mathrm{P}<0.001$, values significantly different from corresponding TA muscle of Ctrl mice by unpaired two-tailed t-test. P7:IKK $\beta$ ca 5 dpi experiment was performed and data was collected in conjunction with P7:TAK1-KO and P7:TAK1-KO;IKK $\beta$ ca mice. Select data presented here is also shown in Figure 4.2. 


\section{FIGURE 3.3}

A.

Ctrl

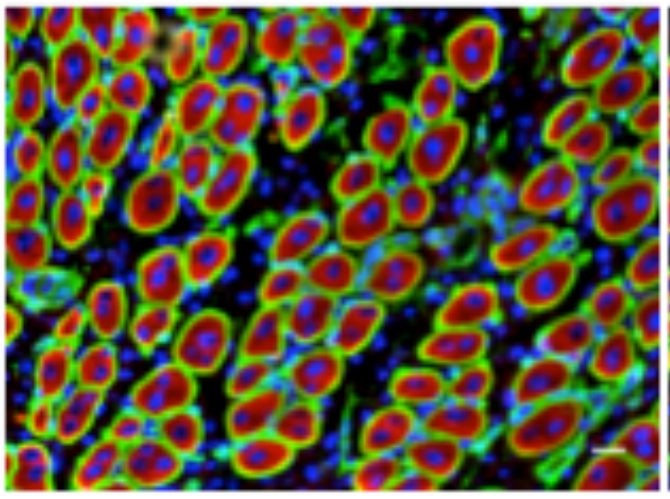

B.

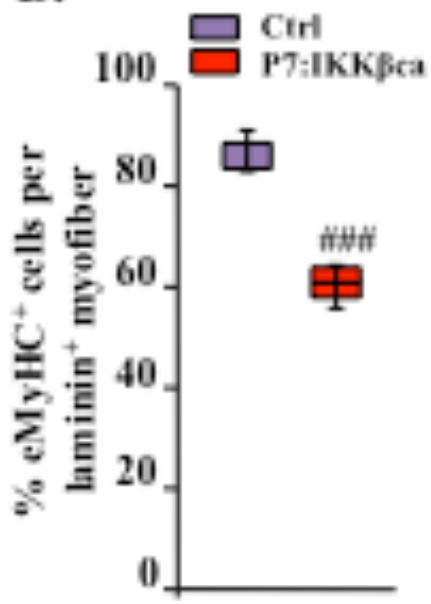

C.

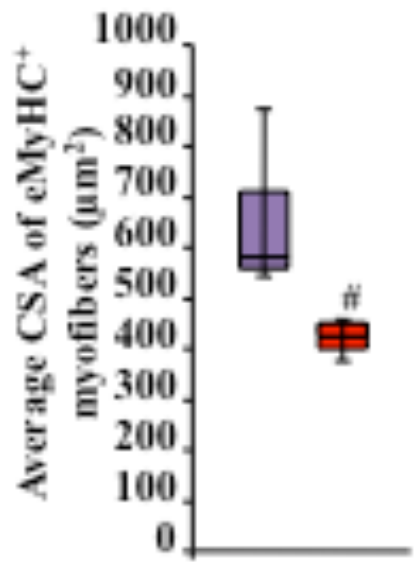

P7:IKKßica

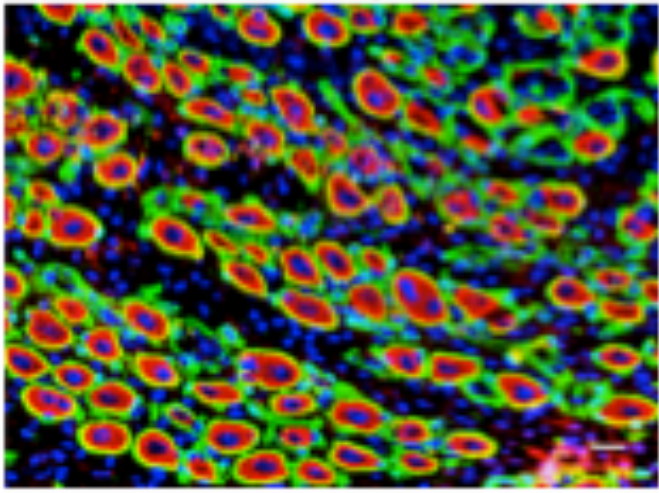

eMyHCILaminin/DAPI

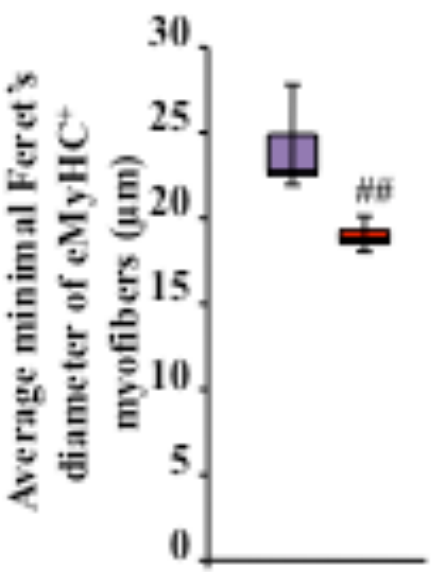


E.

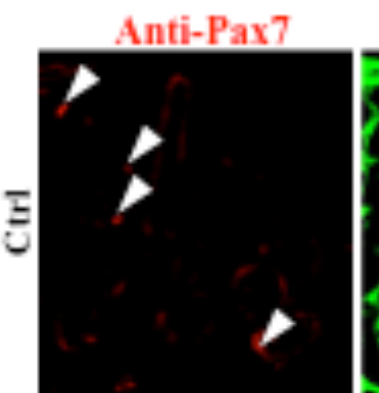

Laminin
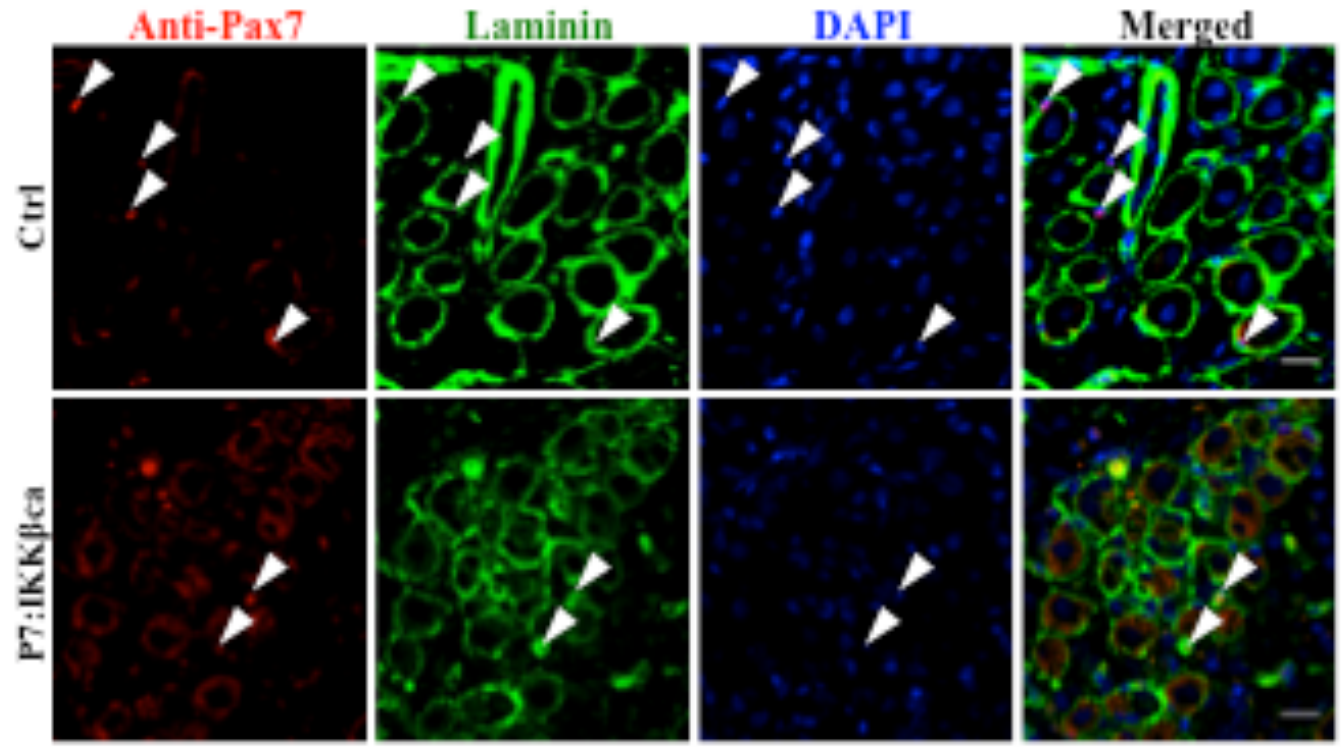

F.

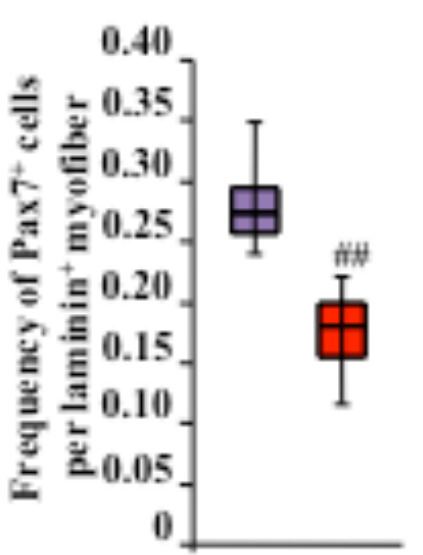

G.

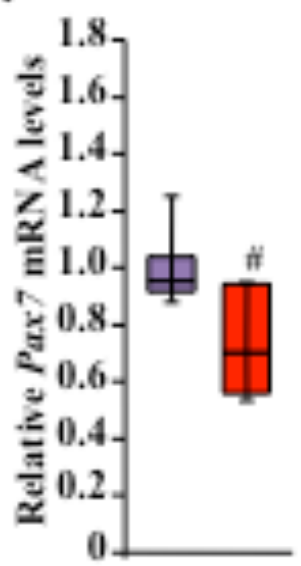

H.

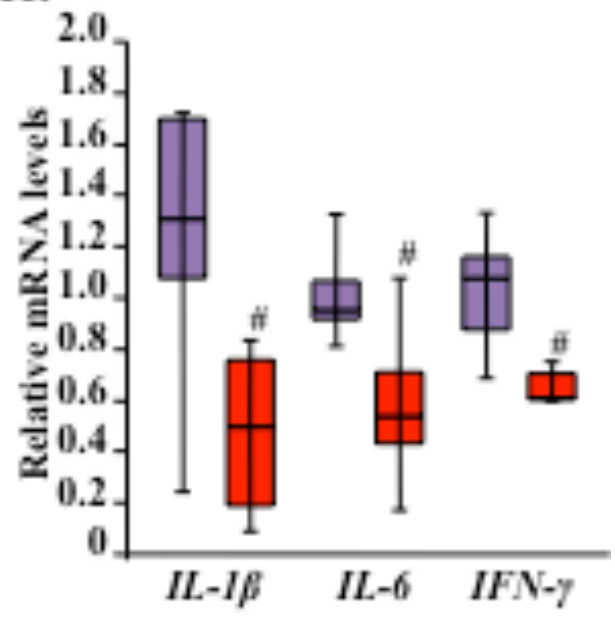




\section{FIGURE 3.3 Overexpression of IKK $\beta$ ca in satellite cells impedes the formation of}

new myofibers and reduces the number of satellite cells. (A) Representative photomicrographs of 5d-injured transverse TA muscle section of $\mathrm{Ctrl}$ and P7:IKK $\beta \mathrm{ca}$ mice after immunostaining for eMyHC and Laminin. Nuclei were identified by staining with DAPI. Scale bar, $20 \mu \mathrm{m}$. Quantification of (B) percentage of eMyHC ${ }^{+}$myofibers per Laminin $^{+}$myofiber, $(\mathbf{C})$ average CSA of eMyHC ${ }^{+}$myofiber, and (D) average minimal Feret's diameter of eMyHC ${ }^{+}$myofiber in 5d-injured TA muscle of P7:IKK $\beta$ ca and Ctrl mice. (E) Representative photomicrograph of 5d-injured transverse TA muscle section of Ctrl and P7:IKK $\beta$ ca mice after immunostaining for Pax7 and Laminin. Nuclei were identified by staining with DAPI. Arrowhead points to Pax $7^{+}$cells. Scale bar, $20 \mu \mathrm{m}$. (F) Quantification of number of $\operatorname{Pax}^{+}$cells per Laminin ${ }^{+}$myofiber. $\mathrm{N}=6$. (G) Relative mRNA levels of Pax7 in 5d-injured TA muscle of P7:IKK $\beta$ ca and Ctrl mice. (H) Relative mRNA levels of various proinflammatory cytokines in 5d-injured TA muscle of P7:IKK $\beta$ ca and Ctrl mice. ${ }^{\#} \mathrm{P}<0.05,{ }^{\# \#} \mathrm{P}<0.01,{ }^{\# \#} \mathrm{P}<0.001$ values significantly different from corresponding TA muscle of Ctrl mice by unpaired two-tailed t-test. 


\section{FIGURE 3.4}

A.

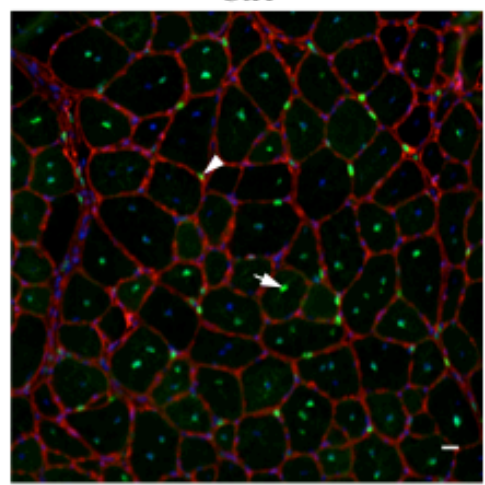

C.

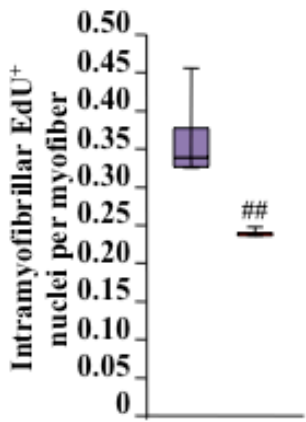

F.

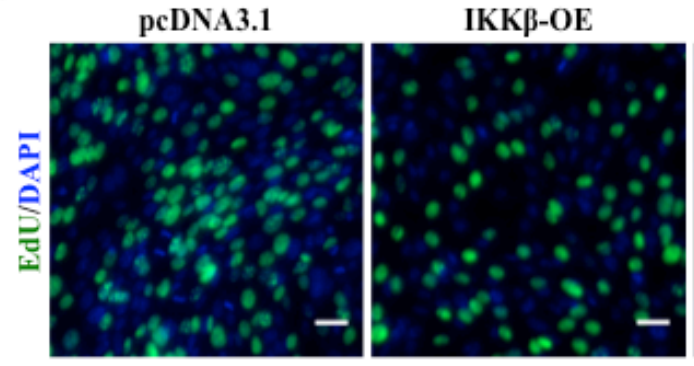

D.
P7:IKKßca
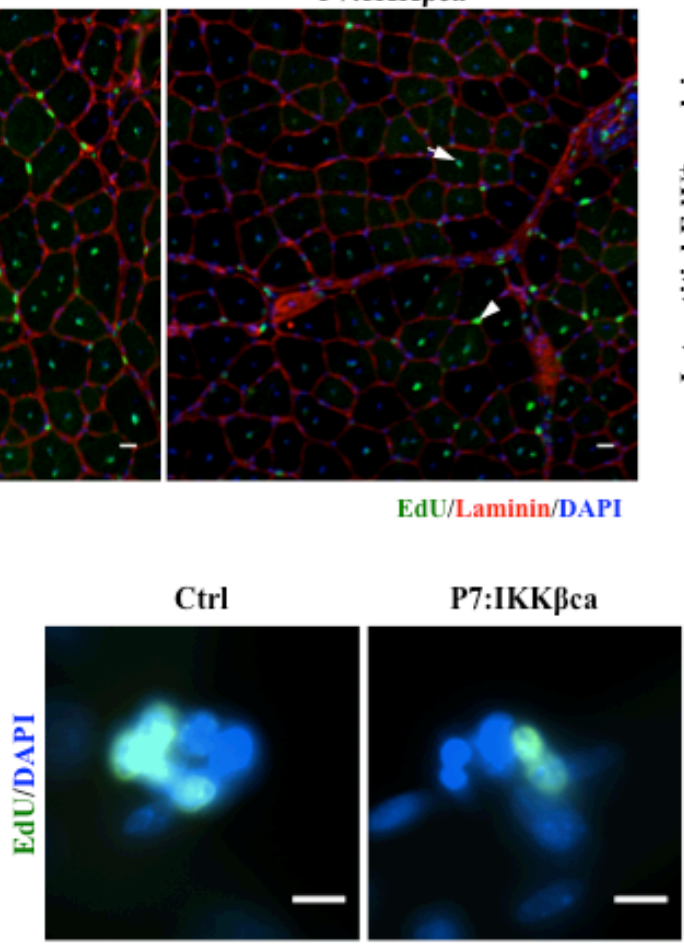

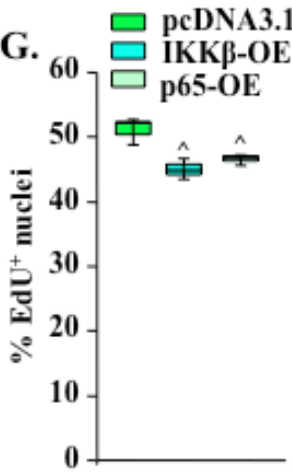

EdU/Laminin/DAPI

E.

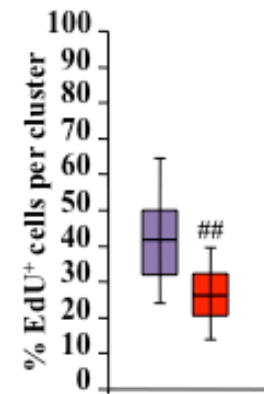

B.
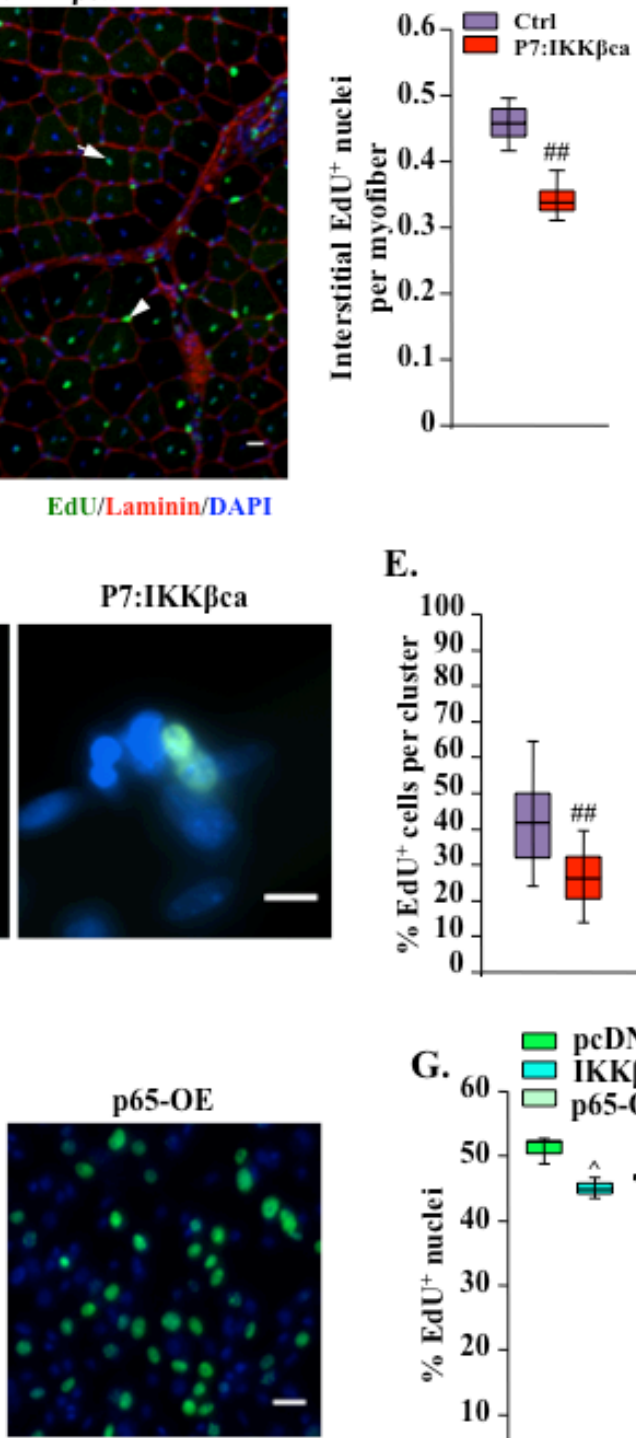


\section{FIGURE 3.4 Overexpression of IKKßca in satellite cells diminishes satellite cell}

proliferation. TA muscle of P7:IKK $\beta$ ca and $\mathrm{Ctrl}$ mice was injured by intramuscular injection of $100 \mu \mathrm{l}$ of $1.2 \% \mathrm{BaCl}_{2}$ solution. After 2 days, the mice were given an intraperitoneal injection of EdU and 12 days later TA muscles were collected and muscle sections prepared were stained to detect EdU, Laminin, and nuclei. (A) Representative photomicrograph of 14d-injured TA muscle section of Ctrl and P7:IKK $\beta$ ca mice after processing for EdU deletion and anti-Laminin staining. Nuclei were identified by staining with DAPI. Arrowhead points to interstitial EdU ${ }^{+}$nuclei. Arrow points to intramyofibrillar EdU ${ }^{+}$nuclei. Scale bar, $20 \mu \mathrm{m}$. Quantification of (B) interstitial $\mathrm{EdU}^{+}$ and $(\mathbf{C})$ intramyofibrillar EdU ${ }^{+}$nuclei per Laminin ${ }^{+}$myofiber in TA muscle of Ctrl and P7:IKK $\beta$ ca mice. $N=4$. Single myofibers cultures were established from EDL muscle of Ctrl and P7:IKK $\beta$ ca mice and cultured for $72 \mathrm{~h}$. The cells were pulse labeled with EdU for last 90 minutes. The myofiber cultures were then stained for EdU. Nuclei were labeled by incubation in DAPI. (D) Representative merged images of EdU and DAPI staining are presented here. Scale bar, $10 \mu \mathrm{m}$. (E) Quantification of percentage of EdU ${ }^{+}$cells per cluster. Cluster was defined as $\geq 4$ cells. $\mathrm{N}=10$-11 fibers per group. (F) Cultured primary myogenic cultures were transfected with vector alone or plasmid vector overexpressing (OE) IKK $\beta$ or p65 cDNA. After 72h, the cells were pulse labelled with EdU for 90 min. Representative merged images of cultures after processing for EdU detection and staining with DAPI. Scale bar, $10 \mu \mathrm{m}$. (G) Quantification of percent EdU ${ }^{+}$cells. $\mathrm{N}=4{ }^{*}{ }^{*} \mathrm{P}<0.05$, ${ }^{\#} \mathrm{P}<0.01$ values significantly different from TA muscle or myofiber suspension culture of Ctrl mice by unpaired two-tailed t-test. ${ }^{\wedge} \mathrm{P}<0.05$, values significantly different from pcDNA3.1 control plasmid by unpaired two-tailed t-test. 
FIGURE 3.5

A.

Ctrl

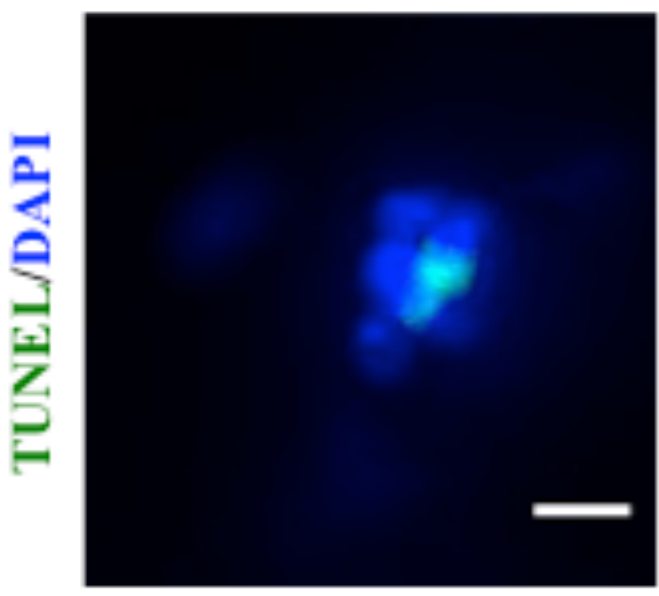

B.

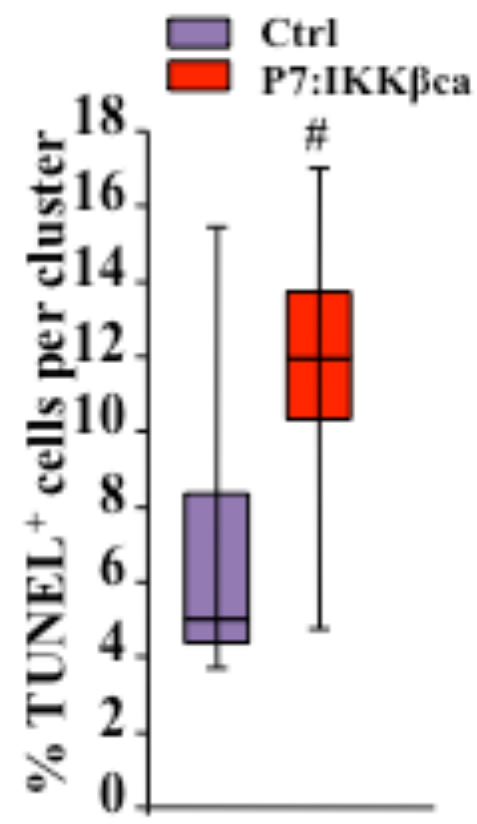

P7:IKKßca

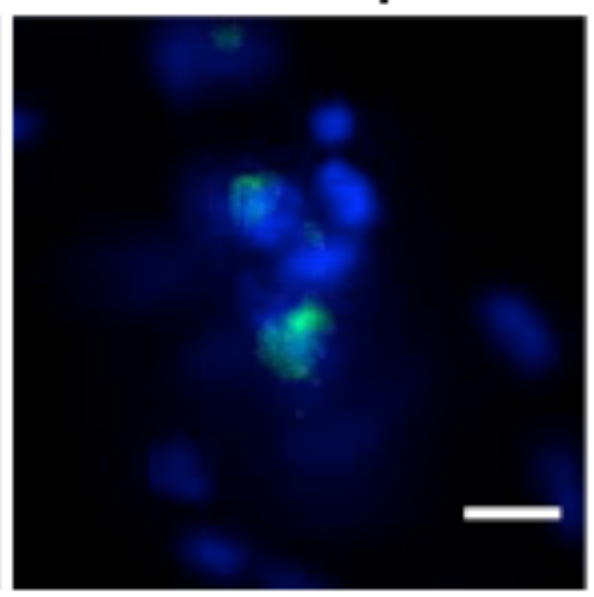

C. $1.4 \square$ pcDNA3.1 $1.2] \square$ p65-OE 1.0 - $1.2- \pm$ 元 击 0.8 응 0.6 $2 \frac{9}{9}$

$\begin{array}{rr} & 0.4 \\ & 0.2\end{array}$ 0 
FIGURE 3.5 Satellite cell-specific overexpression of IKKßca causes an increase in apoptotic cell death. (A) Single myofiber suspension cultures were established from EDL muscle of 6-week old P7:IKK $\beta \mathrm{ca}$ and Ctrl mice, and cultured for $72 \mathrm{~h}$ then processed for detection of TUNEL ${ }^{+}$nuclei. Nuclei were identified by staining with DAPI. Scale bar, $10 \mu \mathrm{m}$. (B) Quantification of percentage of $\mathrm{TUNEL}^{+}$cells per cluster. Cluster was defined as $\geq 4$ cells. $N=10-11$ myofibers per group. (C) Quantification of relative LDH activity (fold change) following IKK $\beta$ and p65 overexpression (OE) plasmid transfection. ${ }^{\#} \mathrm{P}<0.05$, values significantly different from corresponding myofiber suspension culture of Ctrl mice by unpaired two-tailed t-test. ${ }^{\wedge} \mathrm{P}<0.05$, values significantly different from pcDNA3.1 control plasmid by unpaired two-tailed t-test. 
FIGURE 3.6

A.

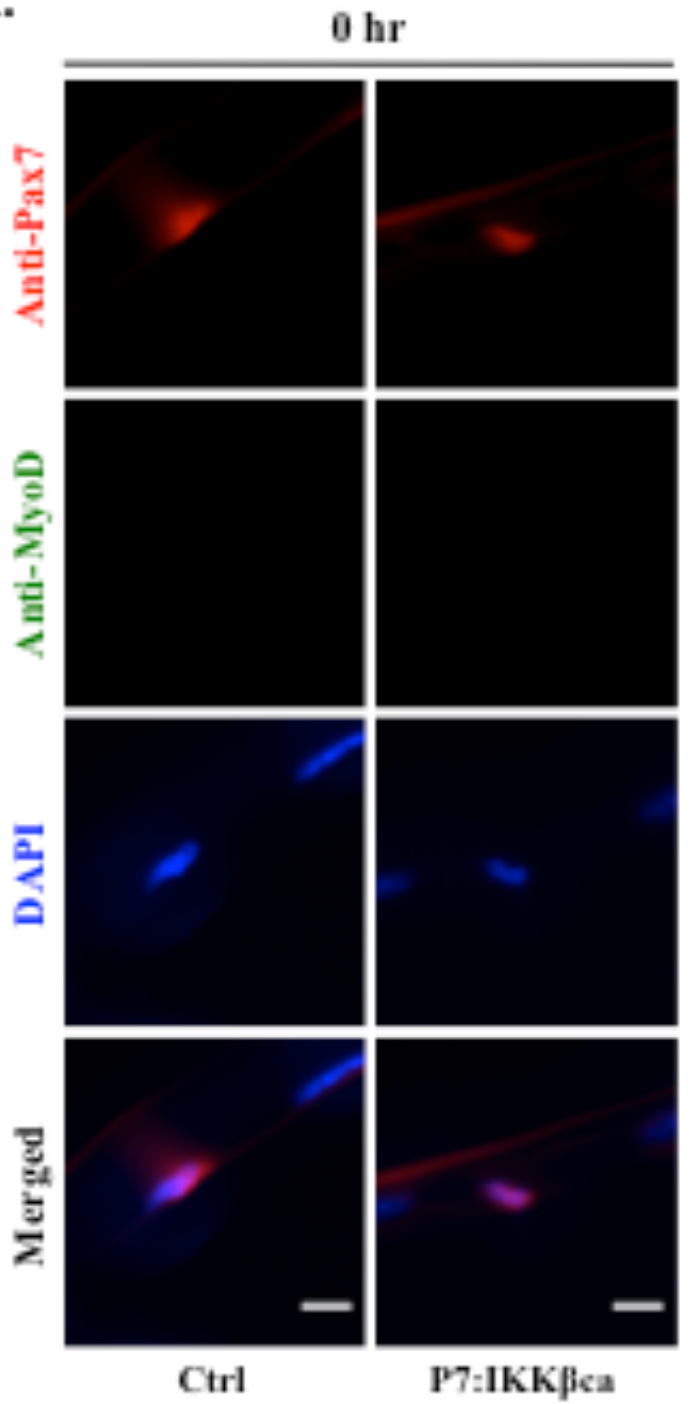

B.
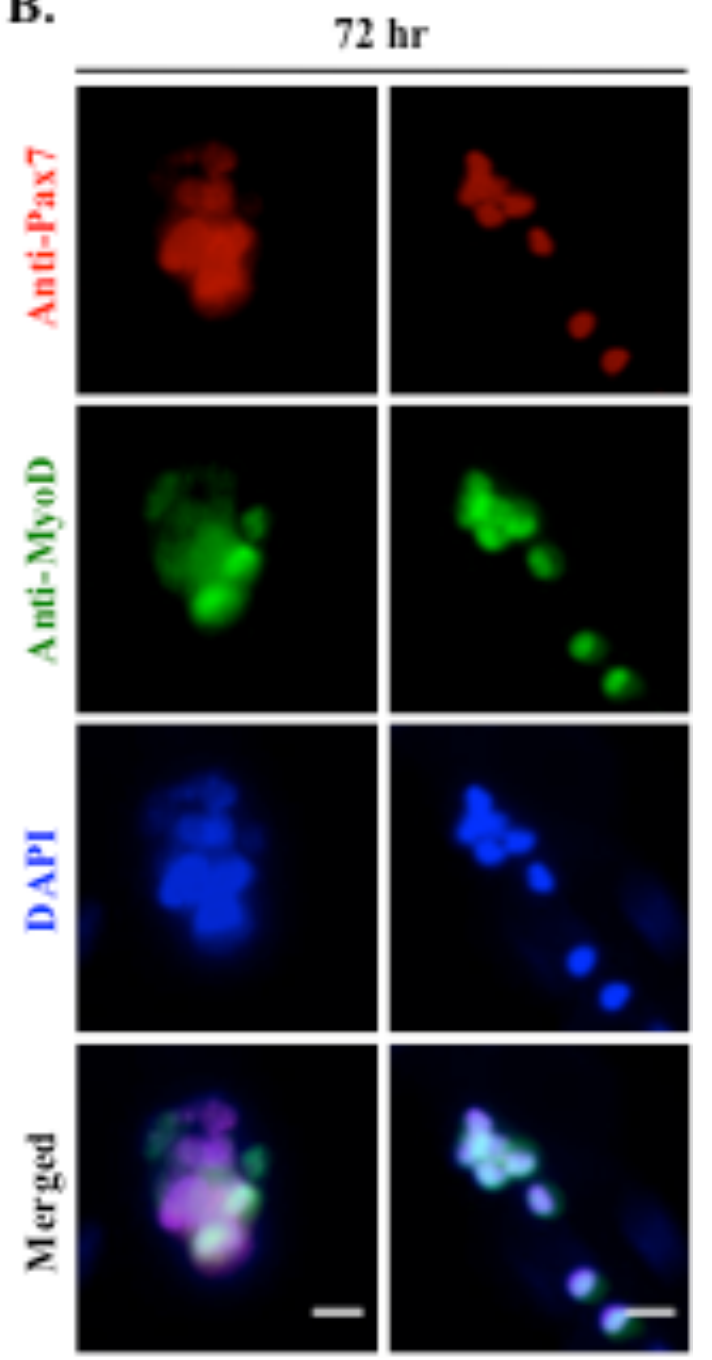

Ctrl

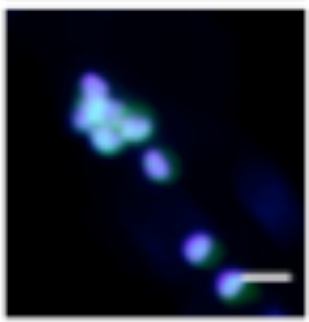

P7:IKK户ica 


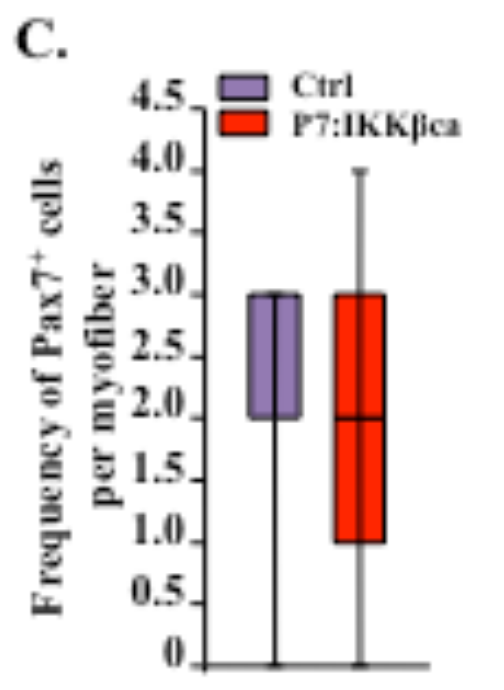

D.

E.

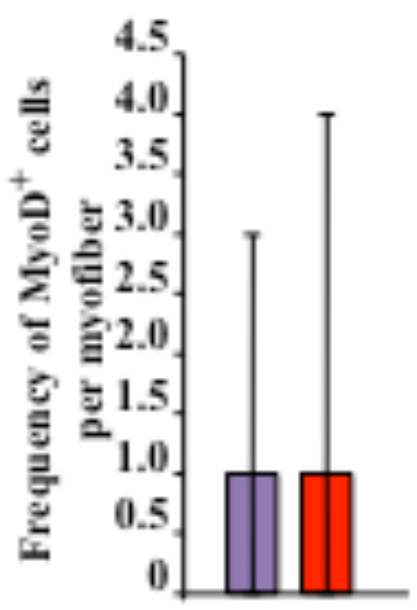

F.

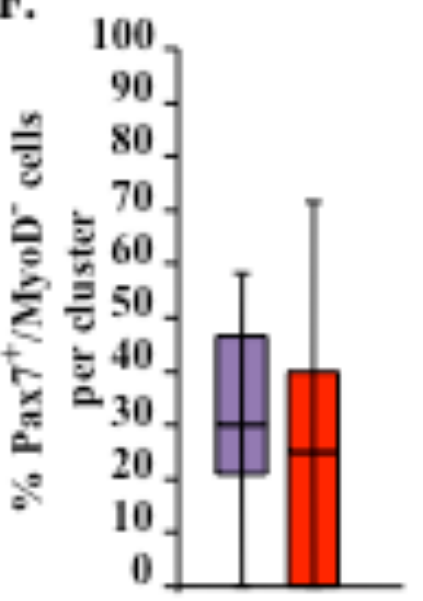

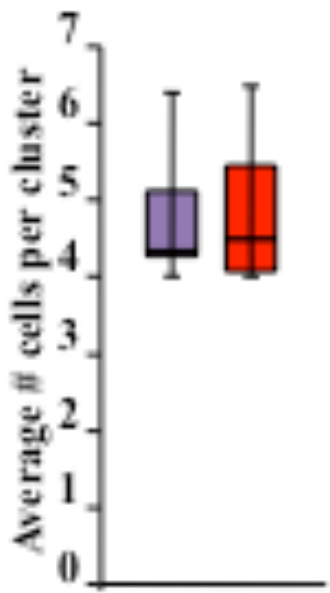

H.

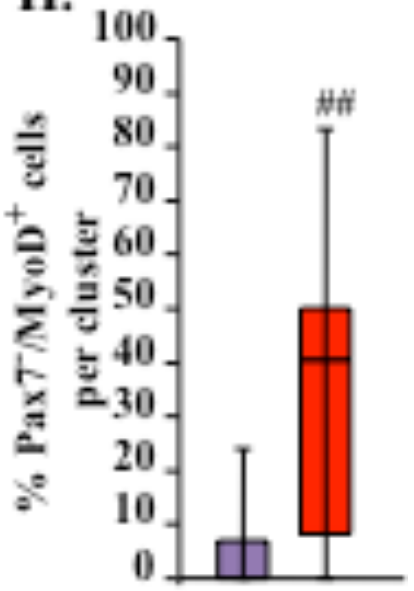


FIGURE 3.6 Satellite cell-specific overexpression of IKKßca results in the precocious differentiation of satellite cells without affecting their self-renewal. Single myofiber suspension cultures were established from EDL muscle of 6-week old P7:IKK $\beta$ ca and Ctrl mice, and cultured for 0 or 72 hours. The cultures were fixed and immunostained for Pax7 and MyoD. Representative individual and merged images of (A) freshly isolated and (B) 72h cultured myofibers from Ctrl and P7:IKK $\beta$ ca mice stained with Pax7, MyoD, and DAPI. Quantification of frequency of $(\mathbf{C})$ Pax $7^{+}$cells per myofiber, and (D) MyoD ${ }^{+}$cells per myofiber in Ctrl and P7:IKK $\beta$ ca a 0h. Quantification of $(\mathbf{E})$ average number of cells per cluster, $(\mathbf{F})$ percentage of $\mathrm{Pax}^{+} / \mathrm{MyoD}^{-}$cells per cluster, (G) percentage of $\mathrm{Pax}^{+} / \mathrm{MyoD}^{+}$cells per cluster, and (H) percentage of $\mathrm{Pax}^{-}$ $/ \mathrm{MyoD}^{+}$cells per cluster in Ctrl and P7:IKK $\beta \mathrm{ca}$ at $72 \mathrm{~h}$ of culturing. Cluster was defined as $\geq 4$ cells. $\mathrm{N}=10-11$ myofibers per group. ${ }^{\#} \mathrm{P}<0.05,{ }^{\# \#} \mathrm{P}<0.01$ values significantly different from corresponding myofiber suspension culture of Ctrl mice by unpaired twotailed t-test. 


\section{FIGURE 3.7}

A.

Anti-Pax 7

Anti-MyoD

DAPI

Merged
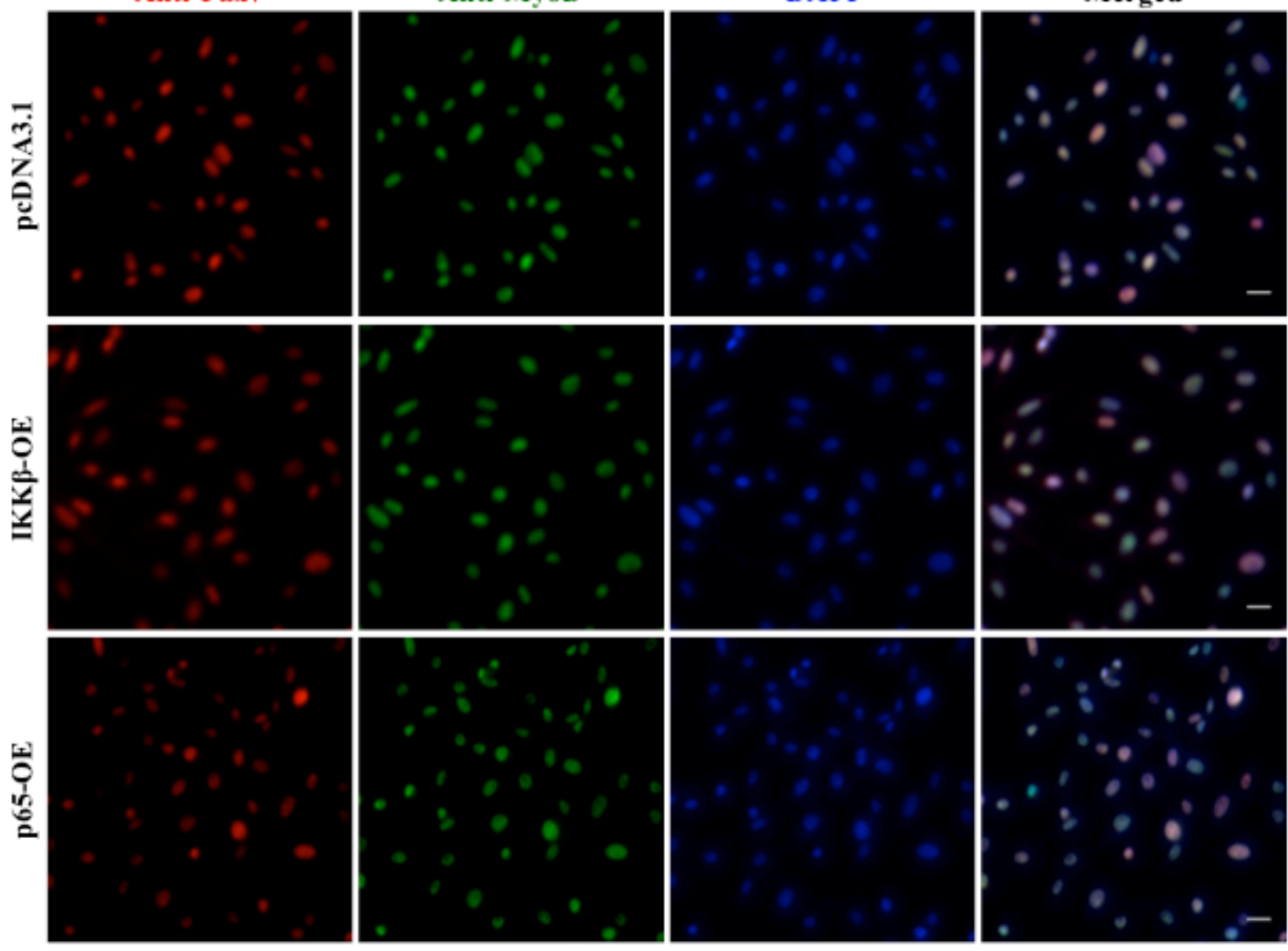

B.
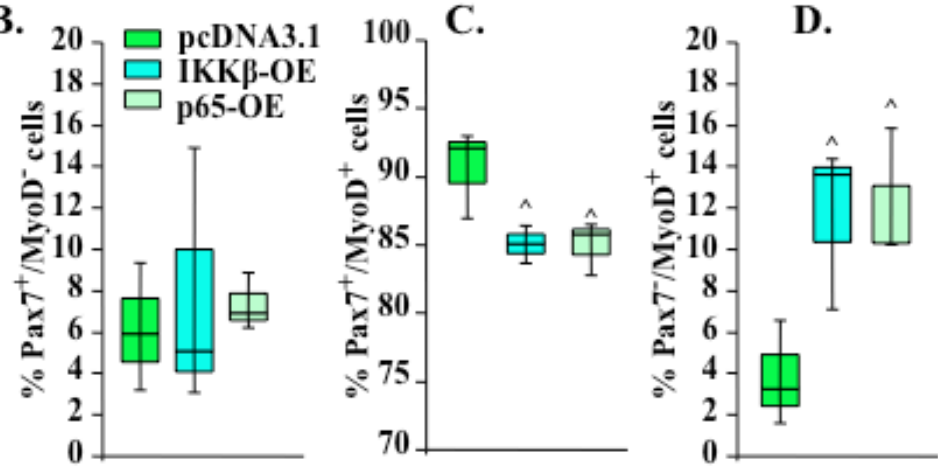


\section{FIGURE 3.7 Overexpression of IKK $\beta$ or p65 result in a precocious differentiation of}

cultured satellite cells. Primary myogenic cultures establish from hind limb muscle of WT mice were transfected with vector alone (i.e. pcDNA3.1) or with wild type IKK $\beta$ or p65 cDNA for 72h. (A) Representative photomicrographs of the cultures after immunostaining for Pax7 and MyoD. Nuclei were identified by staining with DAPI. Quantification of $(\mathbf{B})$ percentage of $\mathrm{Pax}^{+} / \mathrm{MyoD}^{-}$cells, $(\mathbf{C})$ percentage of $\mathrm{Pax} 7^{+} / \mathrm{MyoD}^{+}$ cells, and (D) percentage of Pax 7//MyoD ${ }^{+}$cells in control, IKK $\beta$, or p65-overexpressing (OE) cultures. $\mathrm{N}=3 .{ }^{\wedge} \mathrm{P}<0.05$, values significantly different from pcDNA3.1 control plasmid by unpaired two-tailed t-test. 


\section{CHAPTER 4}

\section{CONSTITUTIVE ACTIVATION OF IKK $\beta$ FAILS TO RESTORE THE REGENERATION DEFICIT CAUSED BY THE SATELLITE CELL-SPECIFIC ABLATION OF TAK1}

4.1 Introduction. A temporal activation of various signaling pathways has been shown to regulate distinct stages of satellite cell-mediated myogenesis. For example, while activation of Notch signaling promotes satellite cell self-renewal, its continued stimulation inhibits differentiation through repressing the expression of MyoD [35]. Moreover, the Wnt7a/Fzd7 planar-cell-polarity pathway drives the symmetric expansion of satellite stem cells during their proliferative phase [68]. On the other hand, the canonical Wnt3a/ $\beta$-catenin pathway has been shown to be involved in regulating satellite cell differentiation and subsequent fusion [68]. It has also been shown that MAPK signaling pathways regulate myogenesis in a context dependent manner [80]. Angiotensin-1/Tie-2 mediated signaling increases the number of quiescent satellite cells through activation of the ERK1/2 signaling pathway [67]. By contrast, activation of p38 MAPK in satellite cells inhibits self-renewal and promotes differentiation [69]. Consistently, work from our lab has shown that the adaptor protein TRAF6 is required for maintaining the quiescent state of un-stimulated satellite cells and promoting their 
proliferation following activation through its downstream targets ERK1/2 and JNK1/2, and through the suppression of p38 MAPK [117]. Moreover, the canonical nuclear factorkappa B (NF-kB) pathway, activated through inhibitor of kappa B kinase- $\beta$ (IKK $\beta)$, promotes the expansion of satellite cells and blocks their terminal differentiation in the context of cancer cachexia [108]. Work from our lab has also shown that sustained activation of canonical NF- $\mathrm{kB}$ signaling in response to TWEAK (a proinflammatory cytokine) treatment diminishes the number Pax $7^{+}$satellite cells in vitro and ex vivo [140]. The findings in Chapter 2 and 3 of this thesis demonstrate that physiological levels of IKK $\beta$ are important for maintaining the survival and proliferation of activated satellite cells in injured muscle, and preventing their precocious differentiation. While the role of canonical NF-kB signaling in satellite cell homeostasis and myogenesis is becoming increasingly evident, the upstream signaling events that dictate its activation status remain less understood.

Transforming growth factor- $\beta$-activated kinase 1 (TAK1) is an important signaling protein of the MEK Kinase family that activates various signaling cascades in response to a variety of stimuli [103]. TAK1-mediated signaling is initiated through its interaction with accessory protein $\mathrm{TAB} 1$, which constitutively binds and activates TAK1 [104]. The TAK1 complex is activated in response to proinflammatory stimuli via K63linked polyubiquitination driven by the $\mathrm{E} 2$ ligase, UBC12/UEV1A, and the RING finger E3 ligases TRAF2 or TRAF6 [105, 106]. K63-linked polyubiquitin chains are then bound by $\mathrm{TAB} 2$ and $\mathrm{TAB} 3$, which have a strong affinity to activate TAK1 through inducing conformational changes in TAK1, leading to its autophosphorylation at Thr187, which initiates the activation of downstream signaling targets, such as NF-kB [106]. Here, 
activated TAK1 phosphorylates IKK $\beta$ on its activation loop, leading to its activation and a subsequent phosphorylation of I $\mathrm{B} \alpha[100,101]$. I $\mathrm{K} \mathrm{B} \alpha$ is then polyubiquitinated through Lys48-linked polyubiquitin chains and degraded by the proteasome [101]. Free from IкB, canonical NF- $\mathrm{kB}$ component RelA/p50 translocates to the nucleus to induce transcription of target genes [101].

We have previously reported that the targeted deletion of TAK1 in satellite cells $\left(\mathrm{TAK}^{\mathrm{scko}}\right.$ ) lead to severe deficits in muscle regeneration following $\mathrm{BaCl}_{2}$-mediated injury [65]. TAK1 was found to be essential for satellite cell proliferation and its inactivation resulted in their precocious differentiation into the myogenic lineage [65]. TAK1-deficient satellite cells exhibit an increase in oxidative stress and necroptosismediated cell death resulting in the depletion of the satellite cell pool [65]. Our findings further revealed that TAK1 regulates satellite cell homeostasis, in part, by activating JNK and canonical NF-אB signaling pathways [65]. Forced expression of constitutively active mutants of JNK and IKK $\beta$ improved the proportion of Pax $7^{+}$satellite cells, blunted oxidative stress, and promoted the survival of TAK1-deficient myogenic cells [65]. However, it remains to be determined whether similar molecular mechanisms are responsible for the poor skeletal muscle regeneration phenotype observed in TAK $1^{\text {scko }}$ mice following muscle injury [65].

Given the proximate hierarchical positioning of TAK1 and the pathway at the center of this thesis; i.e. the canonical NF- $\mathrm{BB}$ signaling pathway, we next sought to investigate weather forced activation of canonical NF- $\mathrm{kB}$ signaling in TAK1-deficient satellite cells would restore proper muscle regeneration in vivo. Through the generation of inducible satellite cell-specific TAK1 knockout, IKK $\beta$ constitutively active mice, we 
demonstrate that constitutive activation of IKK $\beta$ in TAK1-deficient satellite cells does not rescue the regeneration defects observed in P7:TAK1-KO (previously $\mathrm{TAK}^{\text {scko }}$ ) mice following $\mathrm{BaCl}_{2}$ mediated injury.

\subsection{Materials and methods.}

Animals. Satellite cell-specific inducible TAK1-knockout/IKK $\beta$-overexpression mice

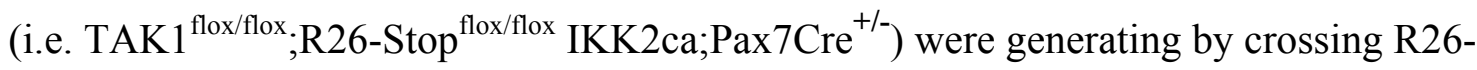
Stop floxed IKK2ca mice with a previously generated satellite cell-specific inducible TAK1-knockout mouse (i.e. P7:TAK1-KO). All mice were in the C57BL/6J background and their genotype was determined by PCR from tail DNA. For Cre-mediated inducible concurrent knockout of TAK1 and overexpression of IKK $\beta$, TAK1 knockout alone, and overexpression of IKK $\beta$ alone, 12-week old mice were injected i.p. with Tamoxifen (10

mg per Kg body weight) for five consecutive days. Control mice were injected with corn oil only. The IACUC and IBC of the University of Louisville approved all experimental protocols with mice in advance.

Skeletal muscle injury. One week after the first injection of tamoxifen, $100 \mu \mathrm{L}$ of $1.2 \%$ $\mathrm{BaCl}_{2}$ (Sigma Chemical Co.) in saline was injected into the TA muscle of mice to induce necrotic muscle injury. At various time points, TA muscle was collected from euthanized mice for biochemical and histological studies.

Histology and morphometric analysis. For skeletal muscle morphology and regeneration assessment, $10 \mu \mathrm{m}$-thick transverse sections of the TA were stained with 
Hematoxylin and Eosin. For quantitative analysis, CSA, minimum Feret's diameter, and multinucleation were analyzed in $\mathrm{H} \& \mathrm{E}$ stained TA muscle sections. H\&E slides were mounted using DPX Mountant For Histology Slide mounting medium (Sigma Chemical Co.) and visualized at room temperature on a Nikon Eclipse TE 2000-U Microscope (Nikon), a digital camera (Nikon Digital Sight DS-Fi1), and Nikon NIS Elements BR 3.00 software (Nikon). Exposure time was kept at $80 \mathrm{~ms}$ and contrast levels were not altered.

Statistical analyses. For the sake of transparency, results were expressed as box-andwhisker plots with the box comprised of the $1^{\text {st }}, 2^{\text {nd }}$, and $3^{\text {rd }}$ quartiles, and the lower and upper whiskers corresponding to the minimum and maximum values, respectively, to display the entire range of data. For experiments involving more than two groups, one way analysis of variance (ANOVA) was performed followed by Tukey's Honest Significant Difference (HSD) post hoc analysis to determine significant differences between groups.

\subsection{Results}

\subsubsection{Satellite cell-specific deletion of IKK $\beta$ fails to rescue the regeneration defects} caused by the specific deletion of TAK1. Stemming from our previous findings where forced expression of a constitutively active mutant of IKK $\beta$ rescued myogenic function in TAK1-deficient primary myoblasts, we aimed to employ a similar genetic approach in

vivo. Accordingly, we crossed TAK $1^{\text {flox/flox }} / \mathrm{Pax}_{7 \mathrm{Cre}}{ }^{+/}$(i.e. P7:TAK1-KO, previously $\mathrm{TAK}^{\text {scko }}$ ) with R26-Stop ${ }^{\text {flox/flox }}$ IKK2ca mice utilized in our investigations in Chapter 3 to 
generate inducible TAK1 ${ }^{\text {flox/flox }} ; \mathrm{R} 26-\mathrm{Stop}^{\text {flox/flox }} \mathrm{IKK} 2 \mathrm{ca} ; \mathrm{Pax} 7 \mathrm{Cre}{ }^{+/-}$mice, henceforth referred to as P7:TAK1-KO;IKK $\beta$ ca mice (Figure 4.1A).

\subsubsection{Forced expression of IKK $\beta$ in TAK1-deficient satellite cells yields no} improvement in regeneration in vivo. Twelve-week-old P7:TAK1-KO only, P7:TAK1KO;IKK $\beta \mathrm{ca}$, and P7:IKK $\beta \mathrm{ca}$ only mice were injected with tamoxifen to induce Crerecombination, or treated with corn oil alone as a vehicle control (TAK1 control, TAK1;IKK $\beta$ control, and IKK $\beta$ control, respectively) (Figure 4.1B). Three days later, TA muscle of the aforementioned 6 groups were given intramuscular injections of $100 \mu 1$ of $1.2 \% \mathrm{BaCl}_{2}$ to induce necrotic muscle injury and regeneration was analyzed at day 5 post-injury. We analyzed the TA muscles through performing $\mathrm{H} \& \mathrm{E}$ staining on transverse sections (Figure 4.2A) and subsequent quantification of: myofiber CSA (Figure 4.2B), minimum Feret's diameter (Figure 4.2C), and percentage of centrally multinucleated myofibers (Figure 4.2D). The regeneration phenotype observed in the P7:TAK1-KO;IKK $\beta$ ca mice resulted in a regeneration pattern that was intermediate between the P7:TAK1-KO mice and the P7:IKK $\beta \mathrm{ca}$, with the P7:TAK1-KO mice showing the largest CSA, minimum Feret's diameter, and percentage of centrally multinucleated fibers. A similar trend was seen between the controls, where the TAK1 control mice showed the largest CSA, minimum Feret's diameter, and percentage of centrally multinucleated fibers, the IKK $\beta$ control showing the smallest values for the aforementioned parameters, and the TAK1;IKK $\beta$ control having values intermediate between the other two controls. Significant difference between groups was determined by one-way ANOVA followed by Tukey's HSD post hoc analysis to determine which 
groups were significantly different from each other. A summary of these findings is found in Figure 4.2B-D. Collectively, these findings demonstrate that constitutive activation of IKK $\beta$ in TAK1-deficient satellite cells does not rescue the muscle regeneration defect brought about by TAK1 deletion.

4.4 Discussion. Satellite cells are muscle stem cells that facilitate muscle repair. Like other adult stem cell, satellite cells retain the capacity to self-renew, through which, a constant pool of healthy cells is maintained. While normally residing in a quiescent state, satellite cells are activated in response to injury or rigorous exercise, where they undergo several rounds of cell division to produce fusion competent myoblasts to support muscle growth and repair. A reduction in the pool of functional satellite cells compromises muscle regeneration and eventually leads to loss of muscle mass, similar to as observed in various genetic muscle disorders.

We have previously reported that the deletion of TAK1 in satellite cells impedes muscle regeneration following injury due to satellite cell dysfunction. Our findings revealed that TAK1 is required for sustaining satellite cell myogenicity through maintaining the expression of Pax7 and supporting satellite cell survival. Overexpression of constitutively active mutant of IKK $\beta$ rescued the expression of Pax7 and abrogated cell death of TAK1-deficient myogenic cultures. In the current study, we aimed to investigate whether a similar approach would yield similar results in vivo and would manifest in efficient muscle regeneration of P7:TAK1-KO muscle. However, unlike our in vitro results, conditional overexpression of constitutively active IKK $\beta$ (i.e. IKK $\beta \mathrm{ca}$ ) in TAK1-knockout satellite cells did not improve muscle regeneration. Defects observed in 
muscle repair were fairly comparable following injury in skeletal muscle of P7:TAK1KO;IKK $\beta$ ca and P7:TAK1-KO mice. While these results may indicate that TAK1 function in satellite cells is independent of $\mathrm{NF}-\kappa \mathrm{B}$ in vivo, further investigations are warranted. It remains to be seen whether the lack of improvement in regenerating muscle of P7:TAK1-KO;IKK $\beta$ ca is merely a result of the supra-physiological activation of the canonical NF- $\mathrm{BB}$ pathway, and not necessarily an absence of hierarchical signaling transduction between TAK1 and IKK $\beta$ in satellite cells of regenerating muscle. Indeed our results from Chapter 3 and accordingly from the current chapter revealed that a heightened activation of the canonical NF- $\kappa$ B pathway via overexpression of IKK $\beta \mathrm{ca}$ in satellite cells impedes muscle regeneration in vivo. Therefore, it is in the realm of possibility that overexpression of a WT form of IKK $\beta$ or the introduction of more physiologically relevant levels of IKK $\beta$ in TAK1-deficient satellite cells would indeed yield an improvement in muscle regeneration. While such investigations would lend important insight into the possible mechanisms through which TAK1 functions in satellite cells in vivo, it is beyond the scope of this thesis, however, will be the subject of future research in our lab.

Altogether, our previous findings complimented with the results of this chapter indicate that the implementation of similar strategies in vitro and in vivo does not necessarily yield comparable outcomes. While a forcible expression of IKK $\beta$ ca restored cell function in TAK1-deficient myogenic cultures, no such improvement was observed in satellite cell-mediated muscle regeneration in P7:TAK1-KO muscle. Forthcoming investigations will elucidate the ambiguity of the aforementioned findings. 


\section{FIGURE 4.1}

A.

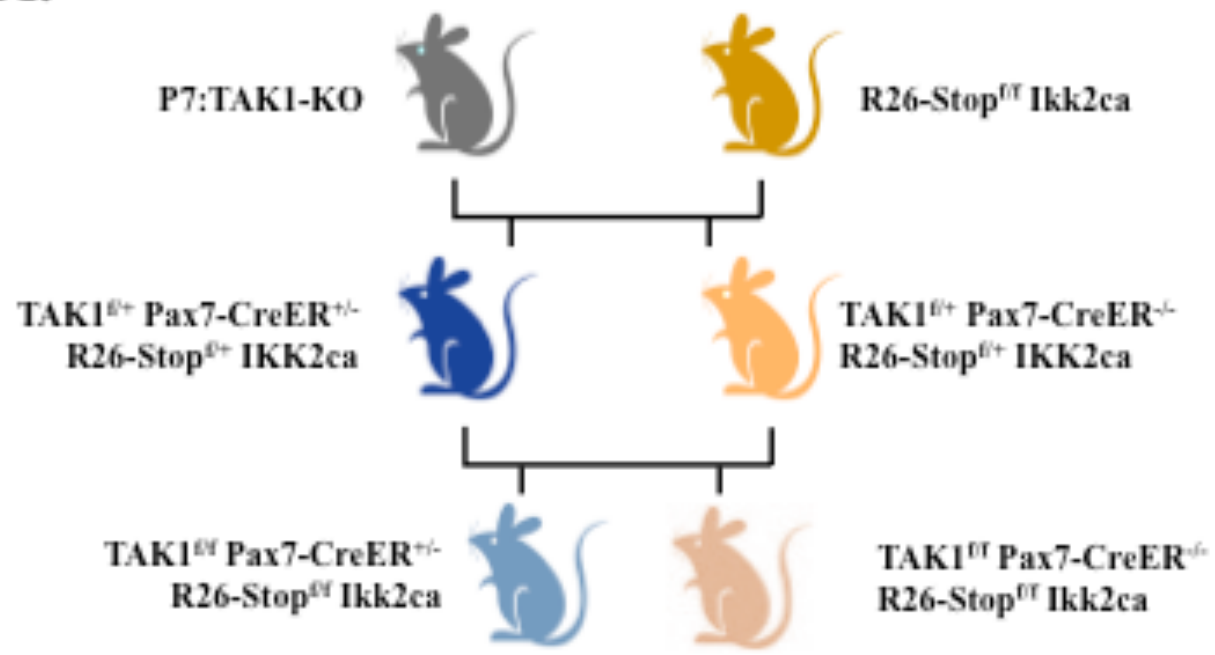

B.

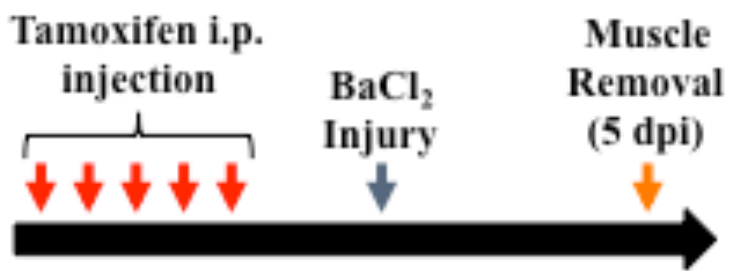

Days 12345678910111213 
FIGURE 4.1 Generation of satellite cell-specific IKKßca overexpressing and Tak1knockout mice. (A) Schematic representation of the breeding strategy used for the generation of concurrent satellite cell-specific TAK1-knockout/IKK $\beta$-overexpression mice. (B) Treatment protocol for tamoxifen-induced Cre recombination and subsequent muscle collection time points in P7:TAK1-KO mice, littermate TAK1 Control, P7:TAK1-KO;IKK $\beta$-CA mice, littermate TAK1;IKK $\beta$ Control mice, P7:IKK $\beta$ ca mice, and IKK $\beta$ Control mice. 


\section{FIGURE 4.2}
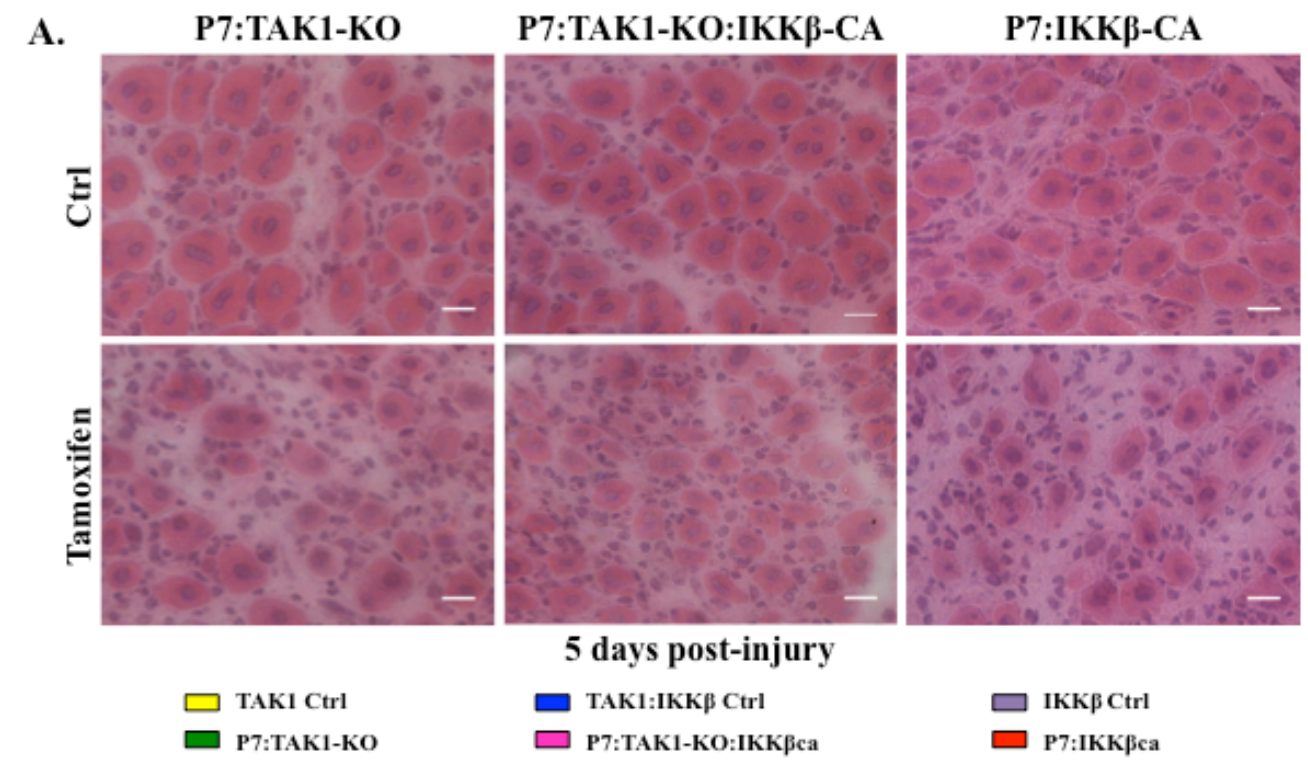

\section{5 days post-injury}

B.

$$
\begin{aligned}
& \square \text { TAK1:IKKß Ctrl } \\
& \square \text { P7:TAK1-KO:IKKßea }
\end{aligned}
$$

C.

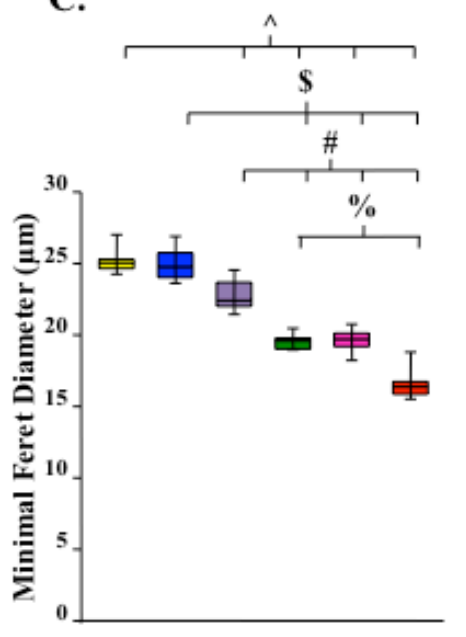

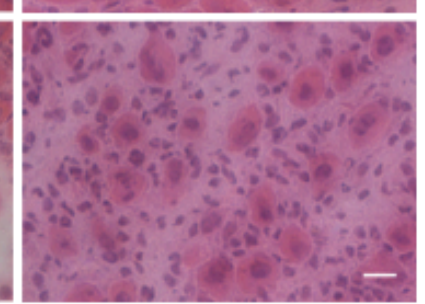

$\square$ ІКК $\mathrm{Ctrl}$

$\square$ P7:IKKßса

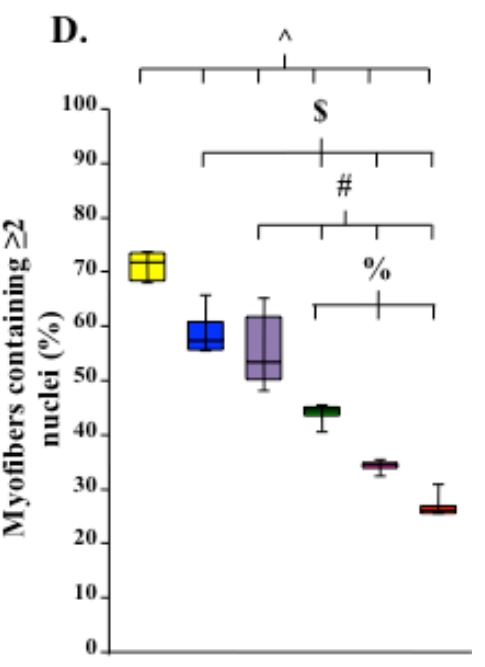




\section{FIGURE 4.2 Overexpression of IKK $\beta$ in satellite cells fails to rescue muscle}

regeneration deficit in TAK1 ${ }^{\text {scko }}$ mice. (A) Representative photomicrographs of H\&Estained sections of 5-days injured TA muscle of 12-week old: P7:TAK1-KO mice, littermate TAK1 Ctrl, P7:TAK1-KO;IKK $\beta$ ca mice, littermate TAK1;IKK $\beta$ Ctrl mice, P7:IKK $\beta$ ca mice, and IKK $\beta$ Ctrl mice following $\mathrm{BaCl}_{2}$-mediated necrotic injury. $\mathrm{N}=5,5$, 4, 4, 6, 6, respectively. Scale bar, $20 \mu \mathrm{m}$. Quantification of (B) average myofiber CSA, (C) minimal Feret's diameter, and (D) the percentage of myofibers containing two or more centrally located nuclei per field at day 5 post-injury. ^^P values significantly different from corresponding TA muscle of TAK1 Ctrl mice by Tukey’s HSD post hoc analysis. ${ }^{\$} \mathrm{P}$ values significantly different from corresponding TA muscle of TAK1;IKK $\beta$ Ctrl mice by Tukey’s HSD post hoc analysis. ${ }^{\#} \mathrm{P}$ values significantly different from corresponding TA muscle of IKK $\beta$ Ctrl mice by Tukey's HSD post hoc analysis. ${ }^{\%} \mathrm{P}$ values significantly different from corresponding TA muscle of P7:TAK1-KO mice by Tukey's HSD post hoc analysis. ${ }^{\&} \mathrm{P}$ values significantly different from corresponding TA muscle of P7:TAK1-KO;IKK $\beta$ ca mice by Tukey's HSD post hoc analysis. P7:IKK $\beta$ ca 5 dpi data experiment was performed and was collected in conjunction with P7:TAK1-KO and P7:TAK1-KO;IKK $\beta$ ca mice. Select data presented here is also shown in Figure 3.2. 


\section{CHAPTER 5}

\section{CONCLUSIONS AND FUTURE WORK}

This chapter is a summary of the work presented in this dissertation. It discusses the implications in satellite cell homeostasis and function with respect to canonical NF- $\mathrm{B}$ signaling. Additionally, it highlights the significance of the findings presented in this dissertation and acknowledges experimental limitations applicable to future research.

5.1 Review of Dissertation. Skeletal muscle is a highly plastic tissue that is capable of repairing itself following physiological insult. The capability of skeletal muscle to regenerate itself following injury is primarily attributed to its satellite stem cell population. Signaling within (un)-injured muscle fibers, as well as in immunological cells that populate the injured skeletal muscle microenvironment, also contribute to various facets of regenerative myogenesis. Through examination of the role of IKK $\beta$, a central kinase of canonical NF- $\mathrm{BB}$ signaling, the findings of this dissertation underscore the complex nature surrounding molecular events that occur during homeostasis of the satellite stem cell and its functional role during regenerative myogenesis. Our results indicate that IKK $\beta$-mediated canonical NF- $\kappa \mathrm{B}$ signaling facilitates the repair process post-injury and maintains the myogenic status of activated satellite cells. 
The findings detailed in Chapter 2 revealed that the ablation of IKK $\beta$ in satellite cells inhibits the process of skeletal muscle regeneration following an acute injury due to a reduction in satellite cell proliferation and survival. Our results suggest that IKK $\beta$ and p65-mediated canonical NF- $\mathrm{KB}$ signaling promotes the survival, proliferation, and selfrenewal of activated satellite cells. Indeed, it appears that satellite cells signal the canonical NF-кB pathway after skeletal muscle injury to increase the satellite stem cell population, and thereby facilitate in the proper regeneration of skeletal muscle. While various inflammatory molecules are expressed following injury, our results suggest that satellite cells do not modulate the expression of these inflammatory cytokines. In various experiments, uninjured skeletal muscle and associated quiescent satellite cells displayed no noticeable phenotype following the satellite cell-specific ablation of IKK $\beta$, suggesting that the canonical NF-KB pathway has a limited impact, if any, on the regulation of quiescent satellite cells.

As discussed in Chapter 3, we explored the effect of supra-physiological activation of the canonical NF- $\mathrm{\kappa B}$ signaling pathway in satellite cells. Since depletion of IKK $\beta$ was deleterious for satellite cell function during regenerative myogenesis, we questioned whether over-stimulation of the canonical NF-кB pathway via IKK $\beta \mathrm{ca}$ overexpression would yield beneficial outcomes. However, this did not turn out to be the case. Similar to as observed with IKK $\beta$ deletion, forced expression of IKK $\beta$ in satellite cells also compromised satellite cell function and impeded muscle regeneration. Indeed these results are consistent with published reports where various myopathic disorders

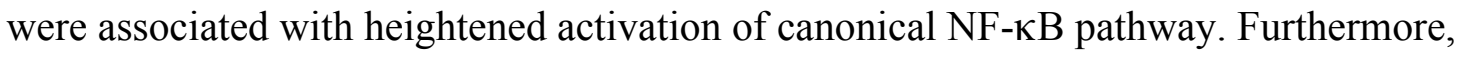
our data suggest that differential aberrations in canonical NF- $\mathrm{KB}$ signaling alter different 
facets of regenerative myogenesis. While both knockout and overexpression of IKK $\beta$ in satellite cells resulted in their precocious differentiation and a reduction in their proliferation and survival, only in the context of the satellite cell-specific IKK $\beta$ deletion did we see a reduction in satellite cell self-renewal. It remains to be determined whether the similar phenotypic outcomes observed from both interventions are result of common upstream mechanisms or occur in a distinct manner.

The focus of Chapter 4 was to investigate whether overexpression of the

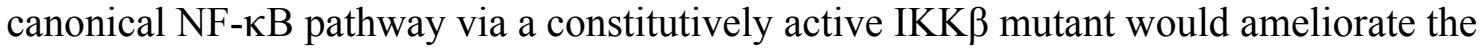
effects of TAK1 deficiency in satellite cells in an in vivo setting. Previous work from our lab revealed a deleterious regeneration phenotype following the specific ablation of TAK1 in satellite cells. Overexpression of a constitutively active mutant form of IKK $\beta$ in vitro rescued myogenic function in TAK1-deficient satellite cell-derived myogenic cultures as evidenced by the restoration of Pax7 expression and an increase in cellular survival and proliferation. However, no such improvement was observed. Regeneration deficits were still evident following the conditional overexpression of IKK $\beta \mathrm{ca}$ in TAK1 knock out satellite cells (P7:TAK1-KO;IKK $\beta \mathrm{ca})$ in vivo, indicating lack of improvement in satellite cell function.

Collectively, the results of this dissertation indicate that canonical NF-KB signaling, mediated by IKK $\beta$, is a versatile modulator of both satellite stem cells and mature skeletal muscle. From our data and taken in conjunction with the current literature, it is becoming increasingly evidenced that a single signaling module may carry distinct functions within various stages of the myogenic lineage. While it has been widely reported that activation of canonical NF- $\mathrm{KB}$ signaling in differentiated muscle is 
associated with virulent outcomes, our findings are suggestive of a supportive role of NF$\kappa \mathrm{B}$ in satellite cell function. However, such role is dictated by a precise regulation of NF$\kappa \mathrm{B}$ activity, as both under- and over-stimulation of the pathway had an adverse effect on the myogenic capacity of satellite cells.

5.2 Scientific contribution and future implementation. The data shown in this dissertation identifies a previously unrecognized role for canonical NF-KB signaling in satellite cell-mediated regenerative myogenesis. Aberrant activation of canonical NF-kB signaling has been observed in various pathological conditions such as Duchenne muscular dystrophy and Rhabdomyosarcoma. Accordingly, therapeutic strategies aimed at abolishing the activity of NF-KB have been implemented in treating such disorders in pre-clinical animal models, and have been reported to be advantageous. However, most studies were focused on evaluating specific disease parameters and were carried out for a short period of time. Such research approaches may result in an oversight of possible deleterious outcomes in various parameters or cell types that do not fall under the evaluation criteria. Furthermore, short-term evaluations may have failed to identify possible virulent outcomes, such as loss of satellite cell function, which may have manifested from NF- $\mathrm{\kappa B}$ inhibition in the long run. The findings from this dissertation emphasize a formerly overlooked biological significance for the canonical NF- $\mathrm{kB}$ signaling in activated satellite cells. As such, our findings underscore the importance of implementing a holistic approach upon evaluating target-based therapies. Therefore, a global long-term evaluation is required upon targeting NF-אB signaling in a myopathic disease setting. 
Our findings from Chapters 2 and 3 reveal that over- and under-stimulation of the canonical NF-кB pathway compromises satellite cell myogenic potential. Furthermore, our findings from Chapter 4 demonstrated that forced activation of canonical NF- $\mathrm{B}$ signaling in TAK1-deficient satellite cells did not improve muscle regeneration in P7:TAK1-KO mice, despite a similar approach yielding beneficial results in vitro. Such outcomes illustrate the importance of a precise regulation of NF- $\mathrm{\kappa B}$ activity within satellite cells to drive proper myogenic function. Exhaustion in satellite cell function and number has been observed in many pathological conditions as well as in age-associated muscle loss. However, the molecular mechanisms that elicit such manifestations remain unknown. It is conceivable that satellite cells cell-autonomous aberration of NF-кB signaling drives the loss of myogenic potential within certain disease contexts. Therefore, reclaiming physiological levels of $\mathrm{NF}-\kappa \mathrm{B}$ activity within satellite cells may prove to be beneficial in treating various muscle disorders.

5.3 Limitations of future implementation. This dissertation concludes in identifying canonical NF- $\kappa \mathrm{B}$ signaling, mediated through IKK $\beta$, as a regulator of the myogenic status of activated satellite cells and a mediator of regeneration of skeletal muscle following injury. However, the work presented here and taken in conjunction with current literature suggests that a cell specific approach would preclude any therapeutic strategies. The ablation of canonical NF- $\mathrm{BB}$ signaling in mature skeletal muscle has been shown to be beneficial for regenerative myogenesis, but the work presented here suggests that it would be deleterious in the satellite stem cell population. While genetic engineering techniques do allow for the conditional deletion/overexpression of various molecules in 
laboratory mouse models, such methodology is not currently available/viable within the realm of translational research. Thus, any pharmacological approaches to inhibiting canonical NF- $\mathrm{kB}$ signaling might have deleterious outcomes in the long term due to the differential effects on stem cells and mature tissues. To elevate the significance of the findings contained within this dissertation, a translational approach allowing for cellspecific alterations in mature tissues types that spared the stem cell population would need to be generated. Lastly, the work in Chapter 4 did not yield a rescue phenotype following the overexpression of IKK $\beta$ in TAK1-deficient satellite cells in vivo, unlike was observed when a similar approach was employed in vitro. It remains to be seen whether this is owed to the supra-physiological activation of the canonical NF- $\mathrm{kB}$ pathway, which was shown to impede satellite cell-mediated muscle regeneration, as was observed in Chapter 3, or if IKK $\beta$ is acting independently of TAK1 in satellite cells in vivo. Along similar lines, in Chapters 3 and 4, the extent to which the canonical NF-kB pathway was activated was not elucidated. Future work should be performed to determine what levels of signaling the canonical NF- $\mathrm{kB}$ pathway produces a therapeutic effect with respect to satellite cell homeostasis and regenerative myogenesis. 


\section{REFERENCES}

1. Trainor PA, Tam PP: Cranial paraxial mesoderm and neural crest cells of the mouse embryo: co-distribution in the craniofacial mesenchyme but distinct segregation in branchial arches. Development (Cambridge, England) 1995, 121(8):2569-2582.

2. Pourquie O: Vertebrate somitogenesis. Annual review of cell and developmental biology 2001, 17:311-350.

3. Maroto M, Bone RA, Dale JK: Somitogenesis. Development (Cambridge, England) 2012, 139(14):2453-2456.

4. Buckingham M, Bajard L, Chang T, Daubas P, Hadchouel J, Meilhac S, Montarras D, Rocancourt D, Relaix F: The formation of skeletal muscle: from somite to limb. J Anat 2003, 202(1):59-68.

5. Hollway GE, Currie PD: Myotome meanderings. Cellular morphogenesis and the making of muscle. EMBO reports $2003,4(9): 855-860$.

6. Innocenzi A, Latella L, Messina G, Simonatto M, Marullo F, Berghella L, Poizat C, Shu CW, Wang JY, Puri PL et al: An evolutionarily acquired genotoxic response discriminates MyoD from Myf5, and differentially regulates hypaxial and epaxial myogenesis. EMBO reports 2011, 12(2):164-171.

7. Parker MH, Seale P, Rudnicki MA: Looking back to the embryo: defining transcriptional networks in adult myogenesis. Nature Reviews Genetics 2003, 4(7):497-507.

8. Kielbowna L, Jedrzejowska I: How is myogenesis initiated in chordates? Folia biologica 2012, 60(3-4):107-119.

9. Grifone R, Kelly RG: Heartening news for head muscle development. Trends in genetics : TIG 2007, 23(8):365-369.

10. Webster MT, Fan CM: c-MET regulates myoblast motility and myocyte fusion during adult skeletal muscle regeneration. PloS one 2013, 8(11):e81757.

11. Griffin CA, Apponi LH, Long KK, Pavlath GK: Chemokine expression and control of muscle cell migration during myogenesis. Journal of cell science 2010, 123(Pt 18):3052-3060. 
12. Mennerich D, Braun T: Activation of myogenesis by the homeobox gene Lbx1 requires cell proliferation. The EMBO journal 2001, 20(24):7174-7183.

13. Yee SP, Rigby PW: The regulation of myogenin gene expression during the embryonic development of the mouse. Genes \& Development 1993, 7(7a):12771289.

14. Andres V, Walsh K: Myogenin expression, cell cycle withdrawal, and phenotypic differentiation are temporally separable events that precede cell fusion upon myogenesis. J Cell Biol 1996, 132(4):657-666.

15. Cusella-De Angelis MG: MyoD, myogenin independent differentiation of primordial myoblasts in mouse somites. The Journal of Cell Biology 1992, 116(5):1243-1255.

16. Rudnicki MA, Schnegelsberg PN, Stead RH, Braun T, Arnold HH, Jaenisch R: MyoD or Myf-5 is required for the formation of skeletal muscle. Cell 1993, 75(7):1351-1359.

17. Halevy O, Piestun Y, Allouh MZ, Rosser BWC, Rinkevich Y, Reshef R, Rozenboim I, Wleklinski-Lee M, Yablonka-Reuveni Z: Pattern of Pax7 expression during myogenesis in the posthatch chicken establishes a model for satellite cell differentiation and renewal. Developmental Dynamics 2004, 231(3):489-502.

18. Hughes SM: Muscle development: Reversal of the differentiated state. Current Biology 2001, 11(6):R237-R239.

19. Yun K, Wold B: Skeletal muscle determination and differentiation: story of a core regulatory network and its context. Current Opinion in Cell Biology 1996, 8(6):877-889.

20. Perry RLS: Molecular mechanisms regulating myogenic determination and differentiation. Frontiers in Bioscience 2000, 5(3):d750-767.

21. Kim JH, Jin P, Duan R, Chen EH: Mechanisms of myoblast fusion during muscle development. Curr Opin Genet Dev 2015, 32:162-170.

22. Mankoo BS, Collins NS, Ashby P, Grigorieva E, Pevny LH, Candia A, Wright $\mathrm{CV}$, Rigby $\mathrm{PW}$, Pachnis $\mathrm{V}$ : Mox2 is a component of the genetic hierarchy controlling limb muscle development. Nature 1999, 400(6739):69-73.

23. Charge SBP: Cellular and Molecular Regulation of Muscle Regeneration. Physiological Reviews 2004, 84(1):209-238.

24. Chen Y, Melton DW, Gelfond JAL, McManus LM, Shireman PK: MiR-351 transiently increases during muscle regeneration and promotes progenitor 
cell proliferation and survival upon differentiation. Physiological Genomics 2012, 44(21):1042-1051.

25. Hindi SM, Kumar A: Toll-like receptor signalling in regenerative myogenesis: friend and foe. The Journal of pathology 2016, 239(2):125-128.

26. Henriques-Pons A, Yu Q, Rayavarapu S, Cohen TV, Ampong B, Cha HJ, Jahnke $\mathrm{V}$, Van der Meulen J, Wang D, Jiang W et al: Role of Toll-like receptors in the pathogenesis of dystrophin-deficient skeletal and heart muscle. Human molecular genetics 2014, 23(10):2604-2617.

27. Tidball JG, Dorshkind K, Wehling-Henricks M: Shared signaling systems in myeloid cell-mediated muscle regeneration. Development (Cambridge, England) 2014, 141(6):1184-1196.

28. Tidball JG, Villalta SA: Regulatory interactions between muscle and the immune system during muscle regeneration. American journal of physiology Regulatory, integrative and comparative physiology 2010, 298(5):R1173-1187.

29. Giordano C, Mojumdar K, Liang F, Lemaire C, Li T, Richardson J, Divangahi M, Qureshi S, Petrof BJ: Toll-like receptor 4 ablation in mdx mice reveals innate immunity as a therapeutic target in Duchenne muscular dystrophy. Human molecular genetics 2015, 24(8):2147-2162.

30. Imai T, Chantry D, Raport CJ, Wood CL, Nishimura M, Godiska R, Yoshie O, Gray PW: Macrophage-derived chemokine is a functional ligand for the $\mathbf{C C}$ chemokine receptor 4. The Journal of biological chemistry 1998, 273(3):17641768 .

31. Krausgruber T, Blazek K, Smallie T, Alzabin S, Lockstone H, Sahgal N, Hussell T, Feldmann M, Udalova IA: IRF5 promotes inflammatory macrophage polarization and TH1-TH17 responses. Nature immunology 2011, 12(3):231238 .

32. Martinez FO, Gordon S: The M1 and M2 paradigm of macrophage activation: time for reassessment. F1000prime reports 2014, 6:13.

33. Kuang S, Kuroda K, Le Grand F, Rudnicki MA: Asymmetric Self-Renewal and Commitment of Satellite Stem Cells in Muscle. Cell 2007, 129(5):999-1010.

34. Bentzinger CF, Wang YX, Rudnicki MA: Building Muscle: Molecular Regulation of Myogenesis. Cold Spring Harbor Perspectives in Biology 2012, 4(2):a008342-a008342.

35. Kuang S, Rudnicki MA: The emerging biology of satellite cells and their therapeutic potential. Trends in Molecular Medicine 2008, 14(2):82-91. 
36. Gussoni E, Soneoka Y, Strickland CD, Buzney EA, Khan MK, Flint AF, Kunkel LM, Mulligan RC: Dystrophin expression in the mdx mouse restored by stem cell transplantation. Nature 1999, 401(6751):390-394.

37. deLapeyriere O, Ollendorff V, Planche J, Ott MO, Pizette S, Coulier F, Birnbaum $\mathrm{D}$ : Expression of the Fgf6 gene is restricted to developing skeletal muscle in the mouse embryo. Development (Cambridge, England) 1993, 118(2):601-611.

38. Floss T, Arnold HH, Braun T: A role for FGF-6 in skeletal muscle regeneration. Genes Dev 1997, 11(16):2040-2051.

39. Cornelison DD, Filla MS, Stanley HM, Rapraeger AC, Olwin BB: Syndecan-3 and syndecan- 4 specifically mark skeletal muscle satellite cells and are implicated in satellite cell maintenance and muscle regeneration. Developmental biology 2001, 239(1):79-94.

40. Lefaucheur JP, Sebille A: Basic fibroblast growth factor promotes in vivo muscle regeneration in murine muscular dystrophy. Neuroscience letters 1995, 202(1-2):121-124.

41. Florini JR, Ewton DZ, Roof SL: Insulin-like growth factor-I stimulates terminal myogenic differentiation by induction of myogenin gene expression. Molecular endocrinology (Baltimore, Md) 1991, 5(5):718-724.

42. Greene EA, Allen RE: Growth factor regulation of bovine satellite cell growth in vitro. Journal of animal science 1991, 69(1):146-152.

43. McFarland DC, Pesall JE, Gilkerson KK: The influence of growth factors on turkey embryonic myoblasts and satellite cells in vitro. General and comparative endocrinology 1993, 89(3):415-424.

44. Barton-Davis ER, Shoturma DI, Sweeney HL: Contribution of satellite cells to IGF-I induced hypertrophy of skeletal muscle. Acta physiologica Scandinavica 1999, 167(4):301-305.

45. Musaro A, McCullagh KJ, Naya FJ, Olson EN, Rosenthal N: IGF-1 induces skeletal myocyte hypertrophy through calcineurin in association with GATA2 and NF-ATc1. Nature 1999, 400(6744):581-585.

46. Semsarian C, Sutrave P, Richmond DR, Graham RM: Insulin-like growth factor (IGF-I) induces myotube hypertrophy associated with an increase in anaerobic glycolysis in a clonal skeletal-muscle cell model. The Biochemical journal 1999, 339 ( Pt 2):443-451.

47. Semsarian C, Wu MJ, Ju YK, Marciniec T, Yeoh T, Allen DG, Harvey RP, Graham RM: Skeletal muscle hypertrophy is mediated by a Ca2+-dependent calcineurin signalling pathway. Nature 1999, 400(6744):576-581. 
48. Erbay E, Park IH, Nuzzi PD, Schoenherr CJ, Chen J: IGF-II transcription in skeletal myogenesis is controlled by mTOR and nutrients. J Cell Biol 2003, 163(5):931-936.

49. Allen RE, Boxhorn LK: Inhibition of skeletal muscle satellite cell differentiation by transforming growth factor-beta. Journal of cellular physiology 1987, 133(3):567-572.

50. Lefaucheur JP, Gjata B, Lafont H, Sebille A: Angiogenic and inflammatory responses following skeletal muscle injury are altered by immune neutralization of endogenous basic fibroblast growth factor, insulin-like growth factor-1 and transforming growth factor-beta 1. Journal of neuroimmunology 1996, 70(1):37-44.

51. Lefaucheur JP, Sebille A: Muscle regeneration following injury can be modified in vivo by immune neutralization of basic fibroblast growth factor, transforming growth factor beta 1 or insulin-like growth factor I. Journal of neuroimmunology 1995, 57(1-2):85-91.

52. McPherron AC, Lawler AM, Lee SJ: Regulation of skeletal muscle mass in mice by a new TGF-beta superfamily member. Nature 1997, 387(6628):83-90.

53. Kirk S, Oldham J, Kambadur R, Sharma M, Dobbie P, Bass J: Myostatin regulation during skeletal muscle regeneration. Journal of cellular physiology 2000, 184(3):356-363.

54. Zarnegar R, Michalopoulos GK: The many faces of hepatocyte growth factor: from hepatopoiesis to hematopoiesis. J Cell Biol 1995, 129(5):1177-1180.

55. Suzuki S, Yamanouchi K, Soeta C, Katakai Y, Harada R, Naito K, Tojo H: Skeletal muscle injury induces hepatocyte growth factor expression in spleen. Biochemical and biophysical research communications 2002, 292(3):709-714.

56. Tatsumi R, Anderson JE, Nevoret CJ, Halevy O, Allen RE: HGF/SF is present in normal adult skeletal muscle and is capable of activating satellite cells. Developmental biology 1998, 194(1):114-128.

57. Tatsumi R, Sheehan SM, Iwasaki H, Hattori A, Allen RE: Mechanical stretch induces activation of skeletal muscle satellite cells in vitro. Experimental cell research 2001, 267(1):107-114.

58. Proto JD, Tang Y, Lu A, Chen WC, Stahl E, Poddar M, Beckman SA, Robbins $\mathrm{PD}$, Nidernhofer LJ, Imbrogno K et al: NF-kappaB inhibition reveals a novel role for HGF during skeletal muscle repair. Cell death \& disease 2015, 6:e1730. 
59. Austin L, Bower J, Kurek J, Vakakis N: Effects of leukaemia inhibitory factor and other cytokines on murine and human myoblast proliferation. Journal of the neurological sciences 1992, 112(1-2):185-191.

60. Bower J, Vakakis N, Nicola NA, Austin L: Specific binding of leukemia inhibitory factor to murine myoblasts in culture. Journal of cellular physiology 1995, 164(1):93-98.

61. Vakakis N, Bower J, Austin L: In vitro myoblast to myotube transformations in the presence of leukemia inhibitory factor. Neurochemistry international 1995, 27(4-5):329-335.

62. Kurek JB, Bower JJ, Romanella M, Koentgen F, Murphy M, Austin L: The role of leukemia inhibitory factor in skeletal muscle regeneration. Muscle \& nerve 1997, 20(7):815-822.

63. Kurek JB, Nouri S, Kannourakis G, Murphy M, Austin L: Leukemia inhibitory factor and interleukin-6 are produced by diseased and regenerating skeletal muscle. Muscle \& nerve 1996, 19(10):1291-1301.

64. Hardee JP, Fix DK, Wang X, Goldsmith EC, Koh HJ, Carson JA: Systemic IL-6 regulation of eccentric contraction-induced muscle protein synthesis. American journal of physiology Cell physiology 2018.

65. Ogura Y, Hindi SM, Sato S, Xiong G, Akira S, Kumar A: TAK1 modulates satellite stem cell homeostasis and skeletal muscle repair. Nature Communications 2015, 6:10123.

66. Hindi SM, Paul PK, Dahiya S, Mishra V, Bhatnagar S, Kuang S, Choi Y, Kumar A: Reciprocal Interaction between TRAF6 and Notch Signaling Regulates Adult Myofiber Regeneration upon Injury. Molecular and Cellular Biology 2012, 32(23):4833-4845.

67. Abou-Khalil R, Le Grand F, Pallafacchina G, Valable S, Authier F-J, Rudnicki MA, Gherardi RK, Germain S, Chretien F, Sotiropoulos A et al: Autocrine and Paracrine Angiopoietin 1/Tie-2 Signaling Promotes Muscle Satellite Cell SelfRenewal. Cell Stem Cell 2009, 5(3):298-309.

68. Le Grand F, Jones AE, Seale V, Scimè A, Rudnicki MA: Wnt7a Activates the Planar Cell Polarity Pathway to Drive the Symmetric Expansion of Satellite Stem Cells. Cell Stem Cell 2009, 4(6):535-547.

69. Bernet JD, Doles JD, Hall JK, Kelly Tanaka K, Carter TA, Olwin BB: p38 MAPK signaling underlies a cell-autonomous loss of stem cell self-renewal in skeletal muscle of aged mice. Nature Medicine 2014, 20(3):265-271. 
70. Shi H, Verma M, Zhang L, Dong C, Flavell RA, Bennett AM: Improved regenerative myogenesis and muscular dystrophy in mice lacking Mkp5. Journal of Clinical Investigation 2013, 123(5):2064-2077.

71. Price FD, von Maltzahn J, Bentzinger CF, Dumont NA, Yin H, Chang NC, Wilson DH, Frenette J, Rudnicki MA: Inhibition of JAK-STAT signaling stimulates adult satellite cell function. Nature Medicine 2014, 20(10):11741181.

72. Ehebauer M, Hayward P, Arias AM: Notch, a Universal Arbiter of Cell Fate Decisions. Science 2006, 314(5804):1414-1415.

73. Bjornson CRR, Cheung TH, Liu L, Tripathi PV, Steeper KM, Rando TA: Notch Signaling Is Necessary to Maintain Quiescence in Adult Muscle Stem Cells. STEM CELLS 2012, 30(2):232-242.

74. Conboy IM: Notch-Mediated Restoration of Regenerative Potential to Aged Muscle. Science 2003, 302(5650):1575-1577.

75. Keshet Y, Seger R: The MAP kinase signaling cascades: a system of hundreds of components regulates a diverse array of physiological functions. Methods in molecular biology (Clifton, NJ) 2010, 661:3-38.

76. Ramos JW: The regulation of extracellular signal-regulated kinase (ERK) in mammalian cells. The international journal of biochemistry \& cell biology 2008, 40(12):2707-2719.

77. Shaul YD, Seger R: The MEK/ERK cascade: from signaling specificity to diverse functions. Biochimica et biophysica acta 2007, 1773(8):1213-1226.

78. Wortzel I, Seger R: The ERK Cascade: Distinct Functions within Various Subcellular Organelles. Genes \& cancer 2011, 2(3):195-209.

79. Yao Z, Seger R: The ERK signaling cascade--views from different subcellular compartments. BioFactors (Oxford, England) 2009, 35(5):407-416.

80. Xie SJ, Li JH, Chen HF, Tan YY, Liu SR, Zhang Y, Xu H, Yang JH, Liu S, Zheng LL et al: Inhibition of the JNK/MAPK signaling pathway by myogenesis-associated miRNAs is required for skeletal muscle development. Cell death and differentiation 2018.

81. Bogoyevitch MA, Ngoei KR, Zhao TT, Yeap YY, Ng DC: c-Jun N-terminal kinase (JNK) signaling: recent advances and challenges. Biochimica et biophysica acta 2010, 1804(3):463-475.

82. Chen F: JNK-induced apoptosis, compensatory growth, and cancer stem cells. Cancer research 2012, 72(2):379-386. 
83. Verma G, Datta M: The critical role of JNK in the ER-mitochondrial crosstalk during apoptotic cell death. Journal of cellular physiology 2012, 227(5):17911795.

84. Coulthard LR, White DE, Jones DL, McDermott MF, Burchill SA: p38(MAPK): stress responses from molecular mechanisms to therapeutics. Trends $\mathrm{Mol} \mathrm{Med}$ 2009, 15(8):369-379.

85. Cuadrado A, Nebreda AR: Mechanisms and functions of p38 MAPK signalling. The Biochemical journal 2010, 429(3):403-417.

86. del Barco Barrantes I, Nebreda AR: Roles of p38 MAPKs in invasion and metastasis. Biochemical Society transactions 2012, 40(1):79-84.

87. Huang G, Shi LZ, Chi H: Regulation of JNK and p38 MAPK in the immune system: signal integration, propagation and termination. Cytokine 2009, 48(3):161-169.

88. Segales J, Perdiguero E, Munoz-Canoves P: Regulation of Muscle Stem Cell Functions: A Focus on the p38 MAPK Signaling Pathway. Frontiers in cell and developmental biology 2016, 4:91.

89. Keren A, Tamir Y, Bengal E: The p38 MAPK signaling pathway: a major regulator of skeletal muscle development. Molecular and cellular endocrinology 2006, 252(1-2):224-230.

90. Liu QC, Zha XH, Faralli H, Yin H, Louis-Jeune C, Perdiguero E, Pranckeviciene E, Munoz-Canoves P, Rudnicki MA, Brand $\mathrm{M}$ et al: Comparative expression profiling identifies differential roles for Myogenin and p38alpha MAPK signaling in myogenesis. Journal of molecular cell biology 2012, 4(6):386-397.

91. Lluis F, Perdiguero E, Nebreda AR, Munoz-Canoves P: Regulation of skeletal muscle gene expression by $\mathbf{p 3 8}$ MAP kinases. Trends Cell Biol 2006, 16(1):3644.

92. von Maltzahn J, Chang NC, Bentzinger CF, Rudnicki MA: Wnt signaling in myogenesis. Trends in Cell Biology 2012, 22(11):602-609.

93. Rawlings JS, Rosler KM, Harrison DA: The JAK/STAT signaling pathway. Journal of cell science 2004, 117(Pt 8):1281-1283.

94. Aaronson DS, Horvath CM: A road map for those who don't know JAKSTAT. Science 2002, 296(5573):1653-1655.

95. Heinrich PC, Behrmann I, Haan S, Hermanns HM, Muller-Newen G, Schaper F: Principles of interleukin (IL)-6-type cytokine signalling and its regulation. The Biochemical journal 2003, 374(Pt 1):1-20. 
96. Kisseleva T, Bhattacharya S, Braunstein J, Schindler CW: Signaling through the JAK/STAT pathway, recent advances and future challenges. Gene 2002, 285(1-2):1-24.

97. O'Shea JJ, Gadina M, Schreiber RD: Cytokine signaling in 2002: new surprises in the Jak/Stat pathway. Cell 2002, 109 Suppl:S121-131.

98. Yang Y, Xu Y, Li W, Wang G, Song Y, Yang G, Han X, Du Z, Sun L, Ma K: STAT3 induces muscle stem cell differentiation by interaction with myoD. Cytokine 2009, 46(1):137-141.

99. McKay BR, Ogborn DI, Baker JM, Toth KG, Tarnopolsky MA, Parise G: Elevated SOCS3 and altered IL-6 signaling is associated with age-related human muscle stem cell dysfunction. American journal of physiology Cell physiology 2013, 304(8):C717-728.

100. Hayden MS: Signaling to NF- B. Genes \& Development 2004, 18(18):21952224.

101. Shih VF-S, Tsui R, Caldwell A, Hoffmann A: A single NFкB system for both canonical and non-canonical signaling. Cell Research 2010, 21(1):86-102.

102. Li H, Malhotra S, Kumar A: Nuclear factor-kappa B signaling in skeletal muscle atrophy. Journal of Molecular Medicine 2008, 86(10):1113-1126.

103. Yamaguchi K, Shirakabe K, Shibuya H, Irie K, Oishi I, Ueno N, Taniguchi T, Nishida E, Matsumoto K: Identification of a Member of the MAPKKK Family as a Potential Mediator of TGF-beta Signal Transduction. Science 1995, 270(5244):2008-2011.

104. Dai L, Aye Thu C, Liu X-Y, Xi J, Cheung PCF: TAK1, more than just innate immunity. IUBMB Life 2012, 64(10):825-834.

105. Fan Y, Yu Y, Mao R, Zhang H, Yang J: TAK1 Lys-158 but not Lys-209 is

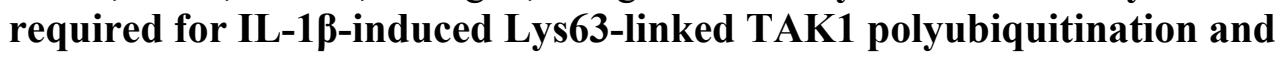
IKK/NF-кB activation. Cellular Signalling 2011, 23(4):660-665.

106. Kanayama A, Seth RB, Sun L, Ea C-K, Hong M, Shaito A, Chiu Y-H, Deng L, Chen ZJ: TAB2 and TAB3 Activate the NF-кB Pathway through Binding to Polyubiquitin Chains. Molecular Cell 2004, 15(4):535-548.

107. Shim JH: TAK1, but not TAB1 or TAB2, plays an essential role in multiple signaling pathways in vivo. Genes \& Development 2005, 19(22):2668-2681.

108. Guttridge DC: NF-kappa B-Induced Loss of MyoD Messenger RNA: Possible Role in Muscle Decay and Cachexia. Science 2000, 289(5488):2363-2366. 
109. Bakkar N, Wang J, Ladner KJ, Wang H, Dahlman JM, Carathers M, Acharyya S, Rudnicki MA, Hollenbach AD, Guttridge DC: IKK/NF-кB regulates skeletal myogenesis via a signaling switch to inhibit differentiation and promote mitochondrial biogenesis. The Journal of Cell Biology 2008, 180(4):787-802.

110. Langen RCJ: Tumor necrosis factor-alpha inhibits myogenic differentiation through MyoD protein destabilization. The FASEB Journal 2004, 18(2):227237.

111. Cai D, Frantz JD, Tawa NE, Melendez PA, Oh B-C, Lidov HGW, Hasselgren PO, Frontera WR, Lee J, Glass DJ et al: IKK及/NF-кB Activation Causes Severe Muscle Wasting in Mice. Cell 2004, 119(2):285-298.

112. Tang Y, Reay DP, Salay MN, Mi MY, Clemens PR, Guttridge DC, Robbins PD, Huard J, Wang B: Inhibition of the IKK/NF-кB pathway by AAV gene transfer improves muscle regeneration in older $\mathbf{m d x}$ mice. Gene Therapy 2010, 17(12):1476-1483.

113. Acharyya S, Villalta SA, Bakkar N, Bupha-Intr T, Janssen PM, Carathers M, Li ZW, Beg AA, Ghosh S, Sahenk Z et al: Interplay of IKK/NF-kappaB signaling in macrophages and myofibers promotes muscle degeneration in Duchenne muscular dystrophy. The Journal of clinical investigation 2007, 117(4):889-901.

114. Dahlman JM, Guttridge DC: Detection of NF-kappaB activity in skeletal muscle cells by electrophoretic mobility shift analysis. Methods in molecular biology (Clifton, NJ) 2012, 798:505-516.

115. Guttridge DC: Signaling pathways weigh in on decisions to make or break skeletal muscle. Current opinion in clinical nutrition and metabolic care 2004, 7(4):443-450.

116. He WA, Berardi E, Cardillo VM, Acharyya S, Aulino P, Thomas-Ahner J, Wang J, Bloomston M, Muscarella P, Nau P et al: NF-kappaB-mediated Pax7 dysregulation in the muscle microenvironment promotes cancer cachexia. The Journal of clinical investigation 2013, 123(11):4821-4835.

117. Hindi SM, Kumar A: TRAF6 regulates satellite stem cell self-renewal and function during regenerative myogenesis. The Journal of clinical investigation 2016, 126(1):151-168.

118. Tilstra JS, Clauson CL, Niedernhofer LJ, Robbins PD: NF-кB in Aging and Disease. Aging and Disease 2011, 2(6):449-465.

119. Monici MC, Aguennouz M, Mazzeo A, Messina C, Vita G: Activation of nuclear factor-kappaB in inflammatory myopathies and Duchenne muscular dystrophy. Neurology 2003, 60(6):993-997. 
120. Messina S, Vita GL, Aguennouz M, Sframeli M, Romeo S, Rodolico C, Vita G: Activation of NF-kappaB pathway in Duchenne muscular dystrophy: relation to age. Acta myologica: myopathies and cardiomyopathies : official journal of the Mediterranean Society of Myology 2011, 30(1):16-23.

121. Cleary MM, Mansoor A, Settelmeyer T, Ijiri Y, Ladner KJ, Svalina MN, Rubin BP, Guttridge DC, Keller C: NFkappaB signaling in alveolar rhabdomyosarcoma. Disease models \& mechanisms 2017, 10(9):1109-1115.

122. Bentzinger CF, Wang YX, Rudnicki MA: Building muscle: molecular regulation of myogenesis. Cold Spring Harb Perspect Biol 2012, 4(2).

123. Relaix F, Zammit PS: Satellite cells are essential for skeletal muscle regeneration: the cell on the edge returns centre stage. Development (Cambridge, England) 2012, 139(16):2845-2856.

124. Yin H, Price F, Rudnicki MA: Satellite cells and the muscle stem cell niche. Physiol Rev 2013, 93(1):23-67.

125. Seale P, Sabourin LA, Girgis-Gabardo A, Mansouri A, Gruss P, Rudnicki MA: Pax 7 is required for the specification of myogenic satellite cells. Cell 2000, 102(6):777-786.

126. von Maltzahn J, Jones AE, Parks RJ, Rudnicki MA: Pax7 is critical for the normal function of satellite cells in adult skeletal muscle. Proceedings of the National Academy of Sciences of the United States of America 2013, 110(41):16474-16479.

127. Hayden MS, Ghosh S: NF-kappaB, the first quarter-century: remarkable progress and outstanding questions. Genes Dev 2012, 26(3):203-234.

128. Kumar A, Takada Y, Boriek AM, Aggarwal BB: Nuclear factor-kappaB: its role in health and disease. $J$ Mol Med (Berl) 2004, 82(7):434-448.

129. Li H, Malhotra S, Kumar A: Nuclear factor-kappa B signaling in skeletal muscle atrophy. $J$ Mol Med 2008, 86(10):1113-1126.

130. Shintaku J, Guttridge DC: Reining in nuclear factor-kappaB in skeletal muscle disorders. Current opinion in clinical nutrition and metabolic care 2013, 16(3):251-257.

131. Mourkioti F, Kratsios P, Luedde T, Song YH, Delafontaine P, Adami R, Parente V, Bottinelli R, Pasparakis M, Rosenthal N: Targeted ablation of IKK2 improves skeletal muscle strength, maintains mass, and promotes regeneration. The Journal of clinical investigation 2006, 116(11):2945-2954.

132. Zhang N, Valentine JM, Zhou Y, Li ME, Zhang Y, Bhattacharya A, Walsh ME, Fischer KE, Austad SN, Osmulski P et al: Sustained NFkappaB inhibition 
improves insulin sensitivity but is detrimental to muscle health. Aging Cell 2017, 16(4):847-858.

133. Bakkar N, Guttridge DC: NF-kappaB signaling: a tale of two pathways in skeletal myogenesis. Physiol Rev 2010, 90(2):495-511.

134. Dogra C, Changotra H, Mohan S, Kumar A: Tumor necrosis factor-like weak inducer of apoptosis inhibits skeletal myogenesis through sustained activation of nuclear factor-kappaB and degradation of MyoD protein. The Journal of biological chemistry 2006, 281(15):10327-10336.

135. Langen RC, Schols AM, Kelders MC, Wouters EF, Janssen-Heininger YM: Inflammatory cytokines inhibit myogenic differentiation through activation of nuclear factor-kappaB. FASEB J 2001, 15(7):1169-1180.

136. Bakkar N, Ladner K, Canan BD, Liyanarachchi S, Bal NC, Pant M, Periasamy M, Li Q, Janssen PM, Guttridge DC: IKKalpha and alternative NF-kappaB regulate PGC-1beta to promote oxidative muscle metabolism. $J$ Cell Biol 2012, 196(4):497-511.

137. Bakkar N, Wang J, Ladner KJ, Wang H, Dahlman JM, Carathers M, Acharyya S, Rudnicki MA, Hollenbach AD, Guttridge DC: IKK/NF-kappaB regulates skeletal myogenesis via a signaling switch to inhibit differentiation and promote mitochondrial biogenesis. J Cell Biol 2008, 180(4):787-802.

138. Enwere EK, Holbrook J, Lejmi-Mrad R, Vineham J, Timusk K, Sivaraj B, Isaac M, Uehling D, Al-Awar R, Lacasse E et al: TWEAK and cIAP1 Regulate Myoblast Fusion Through the Noncanonical NF-kappaB Signaling Pathway. Science signaling 2012, 5(246):ra75.

139. Hindi SM, Shin J, Gallot YS, Straughn AR, Simionescu-Bankston A, Hindi L, Xiong G, Friedland RP, Kumar A: MyD88 promotes myoblast fusion in a cellautonomous manner. Nat Commun 2017, 8(1):1624.

140. Ogura Y, Mishra V, Hindi SM, Kuang S, Kumar A: Proinflammatory cytokine tumor necrosis factor (TNF)-like weak inducer of apoptosis (TWEAK) suppresses satellite cell self-renewal through inversely modulating Notch and NF-kappaB signaling pathways. The Journal of biological chemistry 2013, 288(49):35159-35169.

141. Ballard DW, Dixon EP, Peffer NJ, Bogerd H, Doerre S, Stein B, Greene WC: The 65-kDa subunit of human NF-kappa $B$ functions as a potent transcriptional activator and a target for v-Rel-mediated repression. Proceedings of the National Academy of Sciences of the United States of America 1992, 89(5):18751879 .

142. Geleziunas R, Ferrell S, Lin X, Mu Y, Cunningham ET, Jr., Grant M, Connelly MA, Hambor JE, Marcu KB, Greene WC: Human T-cell leukemia virus type 1 
Tax induction of NF-kappaB involves activation of the IkappaB kinase alpha (IKKalpha) and IKKbeta cellular kinases. Mol Cell Biol 1998, 18(9):51575165 .

143. Riuzzi F, Sorci G, Sagheddu R, Donato R: HMGB1-RAGE regulates muscle satellite cell homeostasis through p38-MAPK- and myogenin-dependent repression of Pax 7 transcription. J Cell Sci 2012, 125(Pt 6):1440-1454.

144. Hindi SM, Paul PK, Dahiya S, Mishra V, Bhatnagar S, Kuang S, Choi Y, Kumar A: Reciprocal interaction between TRAF6 and notch signaling regulates adult myofiber regeneration upon injury. Mol Cell Biol 2012, 32(23):48334845 .

145. Hindi SM, Sato S, Xiong G, Bohnert KR, Gibb AA, Gallot YS, McMillan JD, Hill $\mathrm{BG}$, Uchida $\mathrm{S}$, Kumar A: TAK1 regulates skeletal muscle mass and mitochondrial function. JCI Insight 2018, 3(3).

146. Lepper C, Conway SJ, Fan CM: Adult satellite cells and embryonic muscle progenitors have distinct genetic requirements. Nature 2009, 460(7255):627631.

147. Ogura Y, Hindi SM, Sato S, Xiong G, Akira S, Kumar A: TAK1 modulates satellite stem cell homeostasis and skeletal muscle repair. Nat Commun 2015, 6:10123.

148. Tedesco FS, Dellavalle A, Diaz-Manera J, Messina G, Cossu G: Repairing skeletal muscle: regenerative potential of skeletal muscle stem cells. The Journal of clinical investigation 2010, 120(1):11-19.

149. Kudryashova E, Kramerova I, Spencer MJ: Satellite cell senescence underlies myopathy in a mouse model of limb-girdle muscular dystrophy $2 \mathrm{H}$. The Journal of clinical investigation 2012, 122(5):1764-1776.

150. Shefer G, Van de Mark DP, Richardson JB, Yablonka-Reuveni Z: Satellite-cell pool size does matter: defining the myogenic potency of aging skeletal muscle. Developmental biology 2006, 294(1):50-66.

151. Rinkenbaugh AL, Baldwin AS: The NF-kappaB Pathway and Cancer Stem Cells. Cells 2016, 5(2).

152. Cai D, Frantz JD, Tawa NE, Jr., Melendez PA, Oh BC, Lidov HG, Hasselgren PO, Frontera WR, Lee J, Glass DJ et al: IKKbeta/NF-kappaB activation causes severe muscle wasting in mice. Cell 2004, 119(2):285-298.

153. Dumont NA, Wang YX, Rudnicki MA: Intrinsic and extrinsic mechanisms regulating satellite cell function. Development 2015, 142(9):1572-1581. 
154. Xiong G, Hindi SM, Mann AK, Gallot YS, Bohnert KR, Cavener DR, Whittemore SR, Kumar A: The PERK arm of the unfolded protein response regulates satellite cell-mediated skeletal muscle regeneration. eLife 2017, 6.

155. Zismanov V, Chichkov V, Colangelo V, Jamet S, Wang S, Syme A, Koromilas AE, Crist C: Phosphorylation of eIF2alpha Is a Translational Control Mechanism Regulating Muscle Stem Cell Quiescence and Self-Renewal. Cell Stem Cell 2016, 18(1):79-90.

156. Guttridge DC, Albanese C, Reuther JY, Pestell RG, Baldwin AS, Jr.: NF-kappaB controls cell growth and differentiation through transcriptional regulation of cyclin D1. Mol Cell Biol 1999, 19(8):5785-5799.

157. Lu A, Proto JD, Guo L, Tang Y, Lavasani M, Tilstra JS, Niedernhofer LJ, Wang $\mathrm{B}$, Guttridge DC, Robbins PD et al: NF-kappaB negatively impacts the myogenic potential of muscle-derived stem cells. Molecular therapy : the journal of the American Society of Gene Therapy 2012, 20(3):661-668.

158. Tang Y, Reay DP, Salay MN, Mi MY, Clemens PR, Guttridge DC, Robbins PD, Huard J, Wang B: Inhibition of the IKK/NF-kappaB pathway by AAV gene transfer improves muscle regeneration in older mdx mice. Gene Ther 2010, 17(12):1476-1483.

159. Kumar A, Boriek AM: Mechanical stress activates the nuclear factor-kappaB pathway in skeletal muscle fibers: a possible role in Duchenne muscular dystrophy. FASEB J 2003, 17(3):386-396.

160. Reay DP, Yang M, Watchko JF, Daood M, O'Day TL, Rehman KK, Guttridge DC, Robbins PD, Clemens PR: Systemic delivery of NEMO binding domain/IKKgamma inhibitory peptide to young mdx mice improves dystrophic skeletal muscle histopathology. Neurobiology of disease 2011, 43(3):598-608.

161. Shi H, Verma M, Zhang L, Dong C, Flavell RA, Bennett AM: Improved regenerative myogenesis and muscular dystrophy in mice lacking Mkp5. $J$ Clin Invest 2013, 123(5):2064-2077.

162. Shin J, Tajrishi MM, Ogura Y, Kumar A: Wasting mechanisms in muscular dystrophy. The international journal of biochemistry \& cell biology 2013, 45(10):2266-2279.

163. Sasaki Y, Derudder E, Hobeika E, Pelanda R, Reth M, Rajewsky K, SchmidtSupprian M: Canonical NF-kappaB activity, dispensable for $B$ cell development, replaces BAFF-receptor signals and promotes B cell proliferation upon activation. Immunity 2006, 24(6):729-739. 
164. Otis JS, Niccoli S, Hawdon N, Sarvas JL, Frye MA, Chicco AJ, Lees SJ: Proinflammatory mediation of myoblast proliferation. PloS one 2014, 9(3):e92363.

165. Deyama Y, Takeyama S, Suzuki K, Yoshimura Y, Nishikata M, Matsumoto A: Inactivation of NF-kappaB involved in osteoblast development through interleukin-6. Biochemical and biophysical research communications 2001, 282(5):1080-1084.

166. Yarar-Fisher C, Bickel CS, Kelly NA, Stec MJ, Windham ST, McLain AB, Oster RA, Bamman MM: Heightened TWEAK-NF-kappaB signaling and inflammation-associated fibrosis in paralyzed muscles of men with chronic spinal cord injury. American journal of physiology Endocrinology and metabolism 2016, 310(9):E754-761. 


\section{APPENDICES}

\section{APPENDIX-1}

Sequence of the primers used in qRT-PCR assay.

\begin{tabular}{|r|c|c|}
\hline Gene Name & Forward Primer (5'-3') & Reverse Primer (5'-3') \\
\hline$\beta$-actin & CAG GCA TTG CTG ACA GGA TG & TGC TGA TCC ACA TCT GCT GG \\
\hline IFN- $\gamma$ & GAC AAT CAG GCC ATC AGC AAC & CGG ATG AGC TCA TTG AAT GCT T \\
\hline IL-1 $\beta$ & CTC CAT GAG CTT TGT ACA AGG & TGC TGA TGT ACC AGT TGG GG \\
\hline IL-6 & ATG GCA ATT CTG ATT GTA TG & TGG CTT TGT CTT TCT TGT TA \\
\hline Pax7 & CAG TGT GCC ATC TAC CCA TGC & TTA \\
\hline Myf5 & TGA AGG ATG GAC ATG ACG GAC G & TTG TGT GCT CCG AAG GCT GCT A \\
\hline Myh3 & ACA TCT CTA TGC CAC CTT CGC TAC & GGG TCT TGG TTT CGT TGG GTA T \\
\hline Myh4 & CGG CAA TGA GTA CGT CAC CAA A & TCA AAG CCA GCG ATG TCC AA \\
\hline MyoD & TGG GAT ATG GAG CTT CTA TCG C & GGT GAG TCG AAA CAC GGA TCA T \\
\hline Myogenin & CAT CCA GTA CAT TGA GCG CCT A & GAG CAA ATG ATC TCC TGG GTT G \\
\hline TNF- $\alpha$ & AGC ACA GAA AGC ATG ATC CG & GCC ACA AGC AGG AAT GAG AA \\
\hline
\end{tabular}




\section{APPENDIX-2}

List of abbreviations.

AAV - Adeno-associated Virus

ATP - Adenosine Triphosphate

AV - Annexin V

$\mathrm{BaCl}_{2}-$ Barium Chloride

bFGF - Basic Fibroblast Growth Factor

BMP - Bone Morphogenetic Proteins

BSA - Bovine Serum Albumin

ca - Constitutively Active

cDNA - Complimentary Deoxyribonucleic Acid

c-Met - Tyrosine-Protein Kinase Met

Cre - Cyclase Recombinase

CSA - Cross-Sectional Area

DAPI - 4',6-diamidino-2-phenylindole

DAMP - Damage-associated Molecular Pattern

DMEM - Dulbecco Modified Eagle Medium

DMSO - Dimethyl Sulfoxide

DNA - Deoxyribonucleic Acid

EDL - Extensor Digitorum Longus

EdU - 5-ethynyl-2'-deoxyuridine

eMyHC - Embryonic Myosin Heavy Chain 


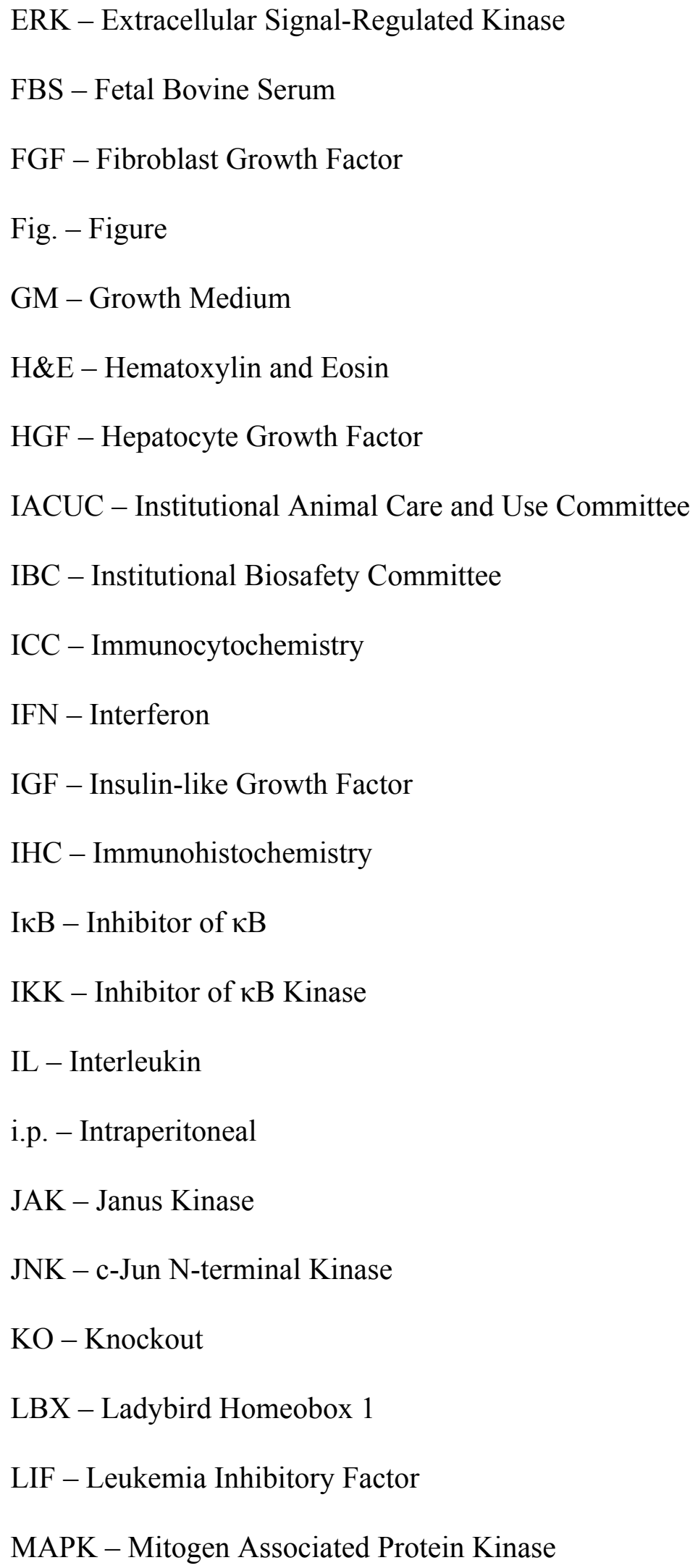


MEK - Mitogen Associated Protein Kinase-Extracellular Signal-Regulated Kinase Mox2 - Mesenchyme Homeobox 2

MRF - Myogenic Regulatory Factor

mTOR - Mechanistic Target of Rapamycin

mRNA - Messenger RNA

MyD88 - Myeloid Differentiation Primary Response 88

Myf - Myogenic Factor

MyHC - Myosin Heavy Chain

MyoD - Myoblast Determination Factor (1)

NF- $\kappa \mathrm{B}-$ Nuclear Factor $-\kappa \mathrm{B}$

NICD - Notch Intracellular Domain

PAMP - Pathogen-associated Molecular Pattern

Pax3 - Paired Box Protein 3

Pax7 - Paired Box Protein 7

PBS - Phosphate Buffered Saline

PCR - Polymerase Chain Reaction

PFA - Paraformaldehyde

PI - Propidium Iodide

qRT-PCR - Quantitative Real-Time Polymerase Chain Reaction

RBP-J $\kappa$ - Recombining Signal Binding Protein for Immunoglobulin $\mathrm{J}_{\kappa}$

RNA - Ribonucleic Acid

STAT - Signal Transducer and Activators of Transcription Protein

TA - Tibialis Anterior 
TAB - TGF- $\beta$ Activated Kinase (MAP3K7) Binding Protein

TAK1 - Transforming Growth Factor $\beta$-Activated Kinase 1

TGF- $\beta$ - Transforming Growth Factor- $\beta$

TIRAP - TIR-Domain Containing Adaptor Protein

TLR - Toll-like Receptor

TNF - Tumor Necrosis Factor

TNFR - Tumor Necrosis Factor Receptor

TRAF6 - TNF Receptor-Associated Factor 6

TRAM - Transduction Associated Membrane Protein

TRIF - TIR-Domain Containing Adaptor-Inducing Interferon- $\beta$

TUNEL - Terminal deoxynucleotidyl transferase dUTP Nick-End Labeling

Wnt - Wingless-Related Integration Site 


\section{APPENDIX-3}

List of antibodies.

\begin{tabular}{|c|c|c|c|}
\hline Antibody & Source and Catalog Number & Dilution & Analysis \\
\hline $\begin{array}{l}\text { Goat Anti-Rabbit IgG, Alexa } \\
\text { Fluor }{ }^{\circledR} 488 \text { conjugated }(\mathrm{H}+\mathrm{L})\end{array}$ & $\begin{array}{l}\text { Thermo Fischer Scientific, A- } \\
11055\end{array}$ & $1: 2000$ & $\mathrm{ICC} / \mathrm{IHC}$ \\
\hline $\begin{array}{l}\text { F(ab')2-Goat Anti-Mouse IgG, } \\
\text { Alexa Fluor }{ }^{\circledR} 594 \text { Cross- } \\
\text { Adsorbed }(\mathrm{H}+\mathrm{L})\end{array}$ & $\begin{array}{l}\text { Thermo Fischer Scientific, A- } \\
11018\end{array}$ & $1: 2000$ & ICC/IHC \\
\hline $\begin{array}{l}\text { Goat Anti-Mouse IgG1, Alexa } \\
\text { Fluor }{ }^{\circledR} 568 \text { conjugated }(\mathrm{H}+\mathrm{L})\end{array}$ & $\begin{array}{l}\text { Thermo Fischer Scientific, A- } \\
21124\end{array}$ & $1: 2000$ & $\mathrm{ICC} / \mathrm{IHC}$ \\
\hline $\begin{array}{l}\text { Goat Anti-Mouse IgG2b, Alexa } \\
\text { Fluor }{ }^{\circledR} 488 \text { conjugated }(H+L)\end{array}$ & $\begin{array}{l}\text { Thermo Fischer Scientific, A- } \\
21141\end{array}$ & $1: 2000$ & ICC/IHC \\
\hline Polyclonal rabbit-anti-BAX & Cell Signaling Technology, 2772 & $1: 1000$ & WB \\
\hline Polyclonal rabbit-anti-Bcl2 & BD Biosciences, 554279 & $1: 1000$ & WB \\
\hline $\begin{array}{l}\text { Monoclonal rabbit-anti-Cleaved } \\
\text { Caspase-3 }\end{array}$ & Cell Signaling Technology, 9664 & $1: 1000$ & WB \\
\hline $\begin{array}{l}\text { Polyclonal rabbit-anti-Cleaved } \\
\text { PARP }\end{array}$ & Cell Signaling Technology, 9544 & $1: 1000$ & WB \\
\hline Polyclonal rabbit-anti-Cyclin A & $\begin{array}{l}\text { Santa Cruz Biotechnology, sc- } \\
596\end{array}$ & $1: 1000$ & WB \\
\hline Polyclonal rabbit-anti-Cyclin D1 & $\begin{array}{l}\text { Santa Cruz Biotechnology, sc- } \\
717\end{array}$ & $1: 1000$ & WB \\
\hline
\end{tabular}




\begin{tabular}{|c|c|c|c|}
\hline Monoclonal mouse-anti-eMyHC & DSHB, F1.652 & $1: 200$ & $\mathrm{IHC}$ \\
\hline $\begin{array}{l}\text { Monoclonal rabbit-anti-phospho- } \\
\text { ІкB } \alpha\end{array}$ & Cell Signaling Technology, 2859 & $1: 500$ & WB \\
\hline Monoclonal rabbit-anti-total IкB $\alpha$ & Cell Signaling Technology, 4812 & $1: 500$ & WB \\
\hline Polyclonal rabbit-anti-total IKK $\beta$ & Cell Signaling Technology, 2684 & $1: 500$ & WB \\
\hline Polyclonal rabbit-anti-Laminin & Sigma, L9393 & $1: 150$ & IHC \\
\hline Monoclonal mouse-anti-MyoD & $\begin{array}{l}\text { Santa Cruz Biotechnology, sc- } \\
377460\end{array}$ & $\begin{array}{l}1: 200, \\
1: 1000\end{array}$ & $\begin{array}{l}\text { ICC, } \\
\text { WB }\end{array}$ \\
\hline Monoclonal mouse-anti-Pax7 & DSHB, pax7 & $\begin{array}{l}1: 100 \\
1: 5\end{array}$ & $\begin{array}{l}\text { WB, } \\
\text { ICC/IHC }\end{array}$ \\
\hline $\begin{array}{l}\text { Monoclonal rabbit-anti-phospho } \\
\text { p65 }\end{array}$ & Cell Signaling Technology, 3033 & $1: 1000$ & WB \\
\hline Monoclonal rabbit-anti-total p65 & Cell Signaling Technology, 8242 & $1: 1000$ & WB \\
\hline $\begin{array}{l}\text { Monoclonal rabbit-anti-alpha- } \\
\text { tubulin }\end{array}$ & Cell Signaling Technology, 2125 & $1: 1000$ & WB \\
\hline
\end{tabular}

ICC - immunocytochemistry; IHC - immunohistochemistry; WB - western blot 


\section{APPENDIX-4}

Camera equipment and settings, exposure time, and contrast modifications

\section{Microscopes:}

ICC/IHC: Nikon TiE 3000 Inverted microscope

H\&E: Nikon Eclipse TE 2000-U microscope

\section{Cameras:}

ICC/IHC: DXM-1200C coded digital camera

H\&E: Nikon Digital Sight DS-Fi1

\section{Acquisition Software: NIS Elements}

Space Resolution:

ICC/IHC: 2560 x 2160; 0.33 microns per pixel @ 20x objective, 0.65 microns per pixel @ 10x objective

H\&E: 2560 x 1920; 0.24 microns per pixel @ 20x objective

Imaging Medium:

ICC/IHC: Aqua-Poly (Polysciences, Inc.)

H\&E: DPX Mountant For Histology Slide (Sigma Chemical Co.)

Exposure and Contrast:

H\&E: 80ms exposure time; no contrast editing

\begin{tabular}{|c|c|c|c|c|c|c|c|c|c|c|}
\cline { 3 - 12 } \multicolumn{2}{c|}{ ICC/IHC } & Settings & \multicolumn{2}{l|}{ TRITC (Red) } & \multicolumn{2}{l|}{ FITC (Green) } & \multicolumn{2}{l|}{ DAPI (Blue) } \\
\hline Image & Objective & Exposure & Low & High & Exposure & Low & High & Exposure & Low & High \\
\hline $\begin{array}{c}\text { Pax7/IKKB } \\
\text { IHC }\end{array}$ & $20 \mathrm{x}$ & $2 \mathrm{sec}$ & 7710 & 42405 & $1.5 \mathrm{sec}$ & 3855 & 53970 & $100 \mathrm{~ms}$ & 3855 & 53970 \\
\hline $\begin{array}{c}\text { Pax7 / p-p65 } \\
\text { ICC }\end{array}$ & $20 \mathrm{x}$ & $300 \mathrm{~ms}$ & 3855 & 42405 & $2 \mathrm{sec}$ & 3855 & 23130 & $80 \mathrm{~ms}$ & 3855 & 53970 \\
\hline $\begin{array}{c}\text { Pax7/p-p65 } \\
\text { IHC }\end{array}$ & $20 \mathrm{x}$ & $2 \mathrm{sec}$ & 3855 & 42505 & $300 \mathrm{~ms}$ & 3855 & 23130 & $80 \mathrm{~ms}$ & 3855 & 53970 \\
\hline $\begin{array}{c}\text { eMyHC/ } \\
\text { Laminin } \\
\text { IHC }\end{array}$ & $20 \mathrm{x}$ & $1 \mathrm{sec}$ & 7710 & 42405 & $200 \mathrm{~ms}$ & 3855 & 50115 & $60 \mathrm{~ms}$ & 7710 & 57825 \\
\hline $\begin{array}{c}\text { Pax7/ } \\
\text { Laminin } \\
\text { IHC }\end{array}$ & $20 \mathrm{x}$ & $2 \mathrm{sec}$ & 7710 & 42405 & $400 \mathrm{~ms}$ & 3855 & 42405 & $80 \mathrm{~ms}$ & 3855 & 42405 \\
\hline $\begin{array}{c}\text { Pax7/ } \\
\text { MyoD ICC }\end{array}$ & $20 \mathrm{x}$ & $2 \mathrm{sec}$ & 7710 & 42405 & $1 \mathrm{sec}$ & 3855 & 42405 & $60 \mathrm{~ms}$ & 3855 & 53970 \\
\hline $\begin{array}{c}\text { EdU } \\
\text { ICC }\end{array}$ & $20 \mathrm{x}$ & $0 \mathrm{sec}$ & 0 & 65536 & $400 \mathrm{~ms}$ & 7710 & 57825 & $60 \mathrm{~ms}$ & 7710 & 57825 \\
\hline $\begin{array}{c}\text { EdU / } \\
\text { Laminin } \\
\text { IHC }\end{array}$ & $10 \mathrm{x}$ & $2 \mathrm{sec}$ & 3855 & 46260 & $2 \mathrm{sec}$ & 3855 & 46260 & $200 \mathrm{~ms}$ & 3855 & 30840 \\
\hline $\begin{array}{c}\text { TUNEL } \\
\text { ICC }\end{array}$ & $20 \mathrm{x}$ & $0 \mathrm{sec}$ & 0 & 65536 & $400 \mathrm{~ms}$ & 7710 & 42405 & $60 \mathrm{~ms}$ & 7710 & 57825 \\
\hline
\end{tabular}


Flurochromes:

1. Alexa Fluor 488 Goat anti-Rabbit IgG $(\mathrm{H}+\mathrm{L})$ : Peak Excitation: 490; Peak Emission: 525

2. Alexa Fluor 546 F(ab')2-Goat anti-Mouse IgG (H+L): Peak Excitation: 556; Peak Emission: 573

3. DAPI: Peak Excitation: 358; Peak Emission: 461

Detailed Specifications for ICC/IHC Imaging:

1. Camera Name: Andor Zyla VSC-00439

2. Numerical Aperture: 0.75 Refractive Index: 1

3. Number of Picture Planes: 3
a. Plane \#1: DAPI
b. Plane \#2: FITC
c. Plane \#3: TRITC

4. Modality: Widefield Fluorescence

5. Camera Settings:

a. Camera Type: Andor Zyla

b. Binning: $1 \mathrm{x} 1$

c. Exposure: See chart on previous page.

d. Readout Mode: Rolling shutter @ 16-bit

e. Readout Rate: $560 \mathrm{MHz}$

i. Conversion Gain: Dual Gain $1 / 4$

f. Spurious Noise Filter: On

i. Sensor Mode: Overlap

g. Trigger Mode: Internal

h. Temperature: $-0.4 \mathrm{C}$

6. Microscope Settings:
a. Microscope: Ti Microscope Nikon Ti
b. Voltage: -1.0
c. Light Path: R100
d. Zoom: $1.00 \mathrm{x}$ 


\section{CURRICULUM VITAE}

\section{Alex R. Straughn, M.S.}

170 Wooded Way

Louisville, KY 40229

Email: arstra03@1ouisville.edu

Phone: 502-851-1800 (Mobile)

\section{A. EDUCATION \& TRAINING:}

(Month/Year)

12/2013: $\quad$ B.S. in Biology

Concentration: Genetics and Subcellular Biology;

University of Louisville, Louisville, KY

07/2017: $\quad$ M.S. in Anatomical Sciences and Neurobiology

Specialization: Skeletal muscle biology and molecular biology; University of Louisville School of Medicine, Louisville KY

08/2018: $\quad$ Ph.D. in Anatomical Sciences and Neurobiology

(Expected) Specialization: Skeletal muscle biology and molecular biology; University of Louisville School of Medicine, Louisville KY

\section{B. PROFESSIONAL EXPERIENCE:}

\section{Graduate Research Assistant}

Department of Anatomical sciences and Neurobiology

University of Louisville School of Medicine, Louisville, KY 40202

2014-2018

\section{Teaching Assistant - Neurobiology}

Department of Anatomical Sciences and Neurobiology

University of Louisville School of Medicine, Louisville, KY 40202

2017

\section{Undergraduate Research Assistant}

Department of Biology

University of Louisville, Louisville, KY 40202

2011-2012 


\section{PEER-REVIEWED PUBLICATIONS:}

1). Hindi SM, Shin J, Gallot YS, Straughn AR, Simionescu-Bankston A, Hindi L, Xiong G, Friedland RP, and Kumar A (2017). MyD88 promotes myoblast fusion in a cellautonomous manner. Nature Communications 8: 1624. DOI: 10.1038/s41467-017-01866W.

2). Gallot YS, McMillan JD, Xiong G, Bohnert KR, Straughn AR, Hill BG, and Kumar A (2017). Distinct roles of TRAF6 and TAK1 in the regulation of adipocyte survival, thermogenesis program, and high-fat diet-induced obesity. Oncotarget, 8:112565112583.

3). Gallot YS, Straughn AR, Bohnert KR, Xiong G, Hindi SM, and Kumar A (2018). MyD88 is required for satellite cell-mediated myofiber regeneration in dystrophindeficient mdx mice. Human Molecular Genetics. (Accepted for publication, in press). https://doi.org/10.1093/hmg/ddy258.

4). Gallot YS, Bohnert KR, Straughn AR, Xiong G, and Kumar A (2018). PERK regulates skeletal muscle mass and contractile function in adult mice. FASEB Journal (Manuscript under revision).

5). Straughn AR, Hindi, S., Xiong, G., and Kumar, A (2018). Canonical NF- $\kappa B$ signaling regulates satellite stem cell homeostasis and function during regenerative myogenesis. (Manuscript under review).

\section{AWARDS AND HONORS:}

- Graduate Fellowship Awarded, University of Louisville, August 2016

- NRSA Institutional Postdoctoral Training (T32) Fellowship Awarded, University of Louisville/James Graham Brown Cancer Center, August 2018

\section{E. POSTER PRESENTATION IN SCIENTIFIC CONFERENCES:}

2017 Dissecting the role of canonical NF- $\mathrm{KB}$ in satellite cell homeostasis and function. Straughn, Alex; Xiong, Guangyan; Kumar, Ashok. Presented at "Research Louisville" meeting in September, 2017, Louisville, KY 